\title{
Tópicos em métodos ótimos para otimização convexa
}

\author{
Diane Rizzotto Rossetto
}

TESE APRESENTADA

$\mathrm{AO}$

Instituto De Matemática e Estatística

DA

Universidade De SÃo Paulo

PARA

OBTENÇÃO DO TÍTULO

$\mathrm{DE}$

DOUTOR EM CIÊNCIAS

\author{
Programa: Matemática Aplicada \\ Orientador: Prof. Dr. Paulo José da Silva e Silva
}

Durante o desenvolvimento deste trabalho o autor recebeu auxílio financeiro do $\mathrm{CNPq}$

São Paulo, março de 2012 


\title{
Tópicos em métodos ótimos para otimização convexa
}

\author{
Este exemplar corresponde à redação \\ final da tese devidamente corrigida \\ e defendida por Diane Rizzotto Rossetto \\ e aprovada pela Comissão Julgadora.
}

Banca Examinadora:

- Prof. Dr. Paulo José da Silva e Silva (orientador) - IME-USP

- Prof. Dr. Carlos Humes Júnior - IME-USP

- Prof. Dr. Clóvis Caesar Gonzaga - UFSC

- Prof. Dr. Roberto Andreani - IMECC-UNICAMP

- Profa. Dra. Fernanda Maria Pereira Raupp - PUC-RIO 
Aos meus pais, Caetano e Terezinha. 


\section{Agradecimentos}

Em primeiro lugar agradeço as pessoas fundamentais na minha vida: meu pai e minha mãe. É tanto amor, tanta compreensão, tanta força que eu recebo de vocês, que nem que passe a vida toda eu vou conseguir retribuir tudo o que vocês me transmitem. Simplesmente amo vocês e agradeço a Deus todos os dias pelo privilégio de ser filha de vocês.

Alguma vez ouvi dizer que os irmãos são a melhor ponte com o seu passado e possivelmente quem vai sempre te apoiar no futuro. Tenho dois anjos na minha vida, meu irmão Diego e minha irmã Daísa. Eles, mesmo que as vezes pareçamos distantes, são as pessoas que sempre me incentivam, me entendem, me chamam a atenção com o máximo de honestidade, confiam em mim e na minha capacidade, enfim, são os melhores amigos que se pode ter. Amo vocês infinitamente...

Tenho muita sorte, pois além de pais maravilhosos, meu pai e minha mãe escolheram pessoas maravilhosas para cuidar de mim. Agradeço muito aos meus padrinhos José e Alice, e toda sua família, pelo cuidado e carinho que depositarem em mim desde bebezinha. Além disto, durante os 10 primeiros anos da minha vida eu tive a companhia da Rosane, até hoje ela me enche de mimos e é uma super confidente. Amo muito todos vocês.

Agradeço a todos familiares que sempre torceram muito por mim. Em especial ao Juca, a Neusa e a toda família do Roger que mesmo diante de momentos de tanta perda nunca perderam a alegria de viver.

No primeiro ano de faculdade eu conheci a Beta. Apesar de todas as diferenças, ela se tornou minha melhor amiga e em 2011 eu tive a honra de ser escolhida como madrinha do seu primeiro filho. O nascimento do Vini na fase final do doutoramento deu mais brilho a minha vida. Beta, Cleidir e Vini, obrigada por serem minha família do coração.

Numa fase do doutorado onde eu via o tempo passar e os resultados não 
saiam eu conheci o Jorge. Não sei se foi o charme de bailarino ou o jeitinho doce, mas ele me inspirou. Meu Amado, obrigada pela paciência, pela compreensão, pela parceria nos momentos de alegria e nos momentos de tensão. Obrigada por ser a pessoa que és, eu te amo muito...

$\mathrm{Na}$ minha casa os animais sempre tiveram um lugar de destaque. Agradeço aos nossos mascotes Dog e Mel, que infelizmente não estão mais entre nós, por toda a alegria que me proporcionaram. Um agradecimento especial a minha companheira de um ano de doutorado aqui em São Paulo, minha cachorrinha Lana.

Desde o início da faculdade eu tive um grande incentivador. Meu irmão do coração Vande, obrigada por sempre acreditar que era possível sair da Unochapecó e chegar aonde eu consegui chegar. Agradeço também aos professores da graduação que sempre me impulsionaram para seguir a diante.

Quando cheguei em Florianópolis para o mestrado tive a sorte de conhecer e conviver com a Meri e a Mari e, através delas conheci a Audina. Meninas muito obrigada pela amizade e pela certeza que mesmo longe sempre estaremos ligadas.

Durante o mestrado convivi com os meninos da "sala dos alunos do Clóvis". Mesmo depois de alguns anos sinto que o sentimento de amizade e coleguismo não se perdeu. Muito obrigada pelo incentivo Gilbertinho, Rafael e Roger.

Agradeço ao meu amigo Borba que desde Floripa me orientava sobre a USP e agora se tornou meu colega de trabalho e de troca de experiências, valeu velho!!!

Quando embarquei nesta "aventura" por São Paulo eu não embarquei sozinha, junto comigo estavam a Priscila e a Luciane. Agradeço a vocês meninas por tudo o que passamos e por tudo o que a convivência com vocês me permitiu aprender.

No final de 2008 precisei sair do apartamento onde vivia e mesmo sem gostar muito da idéia, fui morar numa pensão com muitas pessoas. Foi uma das melhores coisas que aconteceu durante o doutorado. Conheci muitas pessoas e posso afirmar com toda certeza que graças a elas tive uma família aqui em São Paulo. Agradeço a todas, em especial a Vivi, Marcelo, Matheus, Kaluce, Kátia, Normandinho, Dayves, Carolzinha, Leandro, Claudynne, Fabrício, Renan, João, Raoni, Sérgio, Tatá, Tiago e Othon.

Em uma das primeiras vezes que cheguei no IME me orientaram a procurar a sala 257, pois era uma das salas onde os alunos da Aplicada trabalhavam. Lá conheci muitas pessoas, trabalhei muito e também pude me divertir com as pessoas. 
Valeu pessoal do Labmap.

Foi na 257 que conheci dois super amigos: o Daniel e o Nelson. Até mesmo no momento mais complicado do doutorado, eu que estava doente e afastada do IME vocês sempre se fizeram presentes. Graças ao Daniel, conheci a Aymara que, devido a sua espontaneidade e sinceridade, se tornou uma grande amiga. Muitíssimo obrigada, adoro vocês.

Quando cheguei em São Paulo tive a sorte de conviver com um grupo de pesquisa muito sólido e reconhecido não apenas no Brasil, mas também nos grandes centros mundiais de pesquisa. Muito obrigada a todos do grupo de otimização contínua da USP, por tudo o que pude aprender com vocês. Quero fazer um agradecimento especial aos amigos Ricardo e Ellen pela paciência e atenção que sempre tiveram comigo.

Aos colegas e alunos do Centro Universitário SENAC agradeço pelos bons momentos de trabalho. Em especial agradeço a Joyce pela oportunidade desde as épocas das monitorias de MAP2121.

Agradeço aos professores que fizeram parte da banca avaliadora: Paulo, Clóvis, Nino, Fernanda e Carlos pelas valiosas contribuições.

Aos funcionários da CPG que sempre me atenderam com muita atenção e simpatia: Leca, Émerson e Pinho.

Ao CNPQ pelo auxílio financeiro.

A parte final dos agradecimentos esta reservada as pessoas que estão diretamente ligadas ao sucesso deste trabalho. Como eu costumo dizer, ter conhecido o Clóvis mudou o rumo da minha vida e foi através dele que eu conheci profissionais extremamente talentosos e sobretudo seres humanos fantásticos.

Quando cheguei na USP cheia de inseguranças, conheci o Carlos que se tornou um amigo, me orientando sobretudo em questões da minha vida pessoal. Carlos, muito obrigada por toda a atenção e carinho que sempre me tratou.

Através do Clóvis pude conviver com a Elizabeth. Foram muitas idas a Curitiba e Florianópolis. Estas viagens apesar de toda a dureza do trabalho eram sempre muito prazerosas pois a Elizabeth é uma pessoa muito animada e muito otimista. Obrigada por ser uma grande incentivadora. 
Finalmente, quero deixar registrada toda a minha admiração pelos meus orientadores Paulo e Clóvis. Vocês são exemplos de profissionais competentes e totalmente responsáveis pela pesquisa que fazem. Uma das coisas que mais me encanta é a empolgação com que desenvolvem seus trabalhos, isto é muito motivador. Ao Paulo, agradeço muito por ter aceitado me orientar nas condições do ano de 2008 e por toda a paciência que sempre teve comigo. A todos vocês devo lhes dizer muito obrigada por serem ótimos exemplos na minha vida.

Finalizo com uma frase de William Shakespeare que me consolou durante todo o doutorado: Sendo o fim doce, que importa que o início amargo fosse? 


\section{Resumo}

\section{Tópicos em métodos ótimos para otimização convexa}

Neste trabalho apresentamos um novo método ótimo para otimização de uma função convexa diferenciável sujeita a restrições convexas. Nosso método é baseado em ideias de Nesterov e Auslender e Teboulle. A proposta dos últimos autores usa uma distância de Bregman coerciva para garantir que os iterados permaneçam no interior do conjunto viável. Nosso método estende esses resultados para permitir o emprego da distância Euclidiana ao quadrado. Mostramos também como estimar a constante de Lipschitz para o gradiente da função objetivo, o que resulta em uma melhora na eficiência numérica do método. Finalmente, apresentamos experimentos numéricos para validar nossa proposta e comparar com o algoritmo de Nesterov.

Palavras-chave: otimização convexa, métodos ótimos, gradiente Lipschitz contínuo. 


\section{Abstract}

\section{Topics in optimal methods for convex optimization}

In this work we introduce a new optimal method for constrained differentiable convex optimization which is based on previous ideas by Nesterov and Auslender and Teboulle. The method proposed by the last authors use a coercive Bregman distance to ensure that the iterates remain in the interior of the feasible set. Our results extend this method to allow the use of the squared Euclidean distance. We also show how to estimate the Lipschitz constant of the gradient of the objective function, improving the numerical behavior of the method. Finally, we present numerical experiments to validate our approach and compare it to Nesterov's algorithm.

Keywords: convex optimization, optimal methods, gradient Lipschitz continuous. 


\section{Sumário}

Lista de Figuras $\quad$ xiii

$\begin{array}{ll}\text { Introdução } & 1\end{array}$

1 Conceitos Básicos 3

1.1 Funções convexas continuamente diferenciáveis . . . . . . . . . . . . . 6

1.2 Condições de Otimalidade . . . . . . . . . . . . . . . . . . . 10

1.3 Otimalidade de um método . . . . . . . . . . . . . . 12

2 Um método ótimo para problemas sem restrições $\quad 15$

2.1 Ideia do método de Nesterov . . . . . . . . . . . . . . . . . . 15

2.2 Construção da sequência $\left\{\phi_{k}(\cdot)\right\} \ldots$. . . . . . . . . . . . . 19

2.3 Escolhas das sequências que garantem otimalidade . . . . . . . . . . 23

2.4 Otimalidade do método . . . . . . . . . . . . . . . . . . 29

3 Métodos para problemas com restrições 33

3.1 O método de Nesterov . . . . . . . . . . . . . . . . . . . 33

3.1 .1 Mapeamento Gradiente . . . . . . . . . . . . . 34

3.2 O método de Auslender e Teboulle . . . . . . . . . . . . . . . . 35

3.2.1 Distâncias de Bregman Coercivas . . . . . . . . . . . . 37

3.2.2 Construção da $\left\{\phi_{k}(\cdot)\right\}$ usando distâncias de Bregman coercivas 40

3.3 Generalização para projeções não interiores . . . . . . . . . . . . . . 47

3.3.1 Tentativa de aumentar o decréscimo da função objetivo . . . . 52

3.3.2 L adaptativo . . . . . . . . . . . . . . . 55

4 Testes Numéricos $\quad 63$

4.1 Detalhes de implementação. . . . . . . . . . . . . . . . 63

4.1.1 Problemas testados . . . . . . . . . . . . . . 63

4.1 .2 Critério de Comparações . . . . . . . . . . . . . . . . . . 64

4.1.3 Critério de Parada . . . . . . . . . . . . . 65

4.2 Comparações . . . . . . . . . . . . . . . 66 
4.2.1 Critério de aceitação para $L_{a} \quad \ldots \ldots$. . . . . . . . . . . 66

4.2.2 Escolha da distância de Bregman . . . . . . . . . . . . 66

4.2.3 Como determinar novos pontos . . . . . . . . . . 70

4.2.4 Introduzindo o passo SPG . . . . . . . . . . . 75

$\begin{array}{lr}\text { Considerações Finais } & 87\end{array}$

Referências Bibliográficas $\quad 89$ 


\section{Lista de Figuras}

1.1 Conjunto Convexo . . . . . . . . . . . . . . . . . . . . . 4

1.2 Função Convexa . . . . . . . . . . . . . . . . . . . . . 4

1.3 Função Convexa Diferenciável . . . . . . . . . . . . . . . . . 7

$1.4 f(x)=|x| \ldots \ldots \ldots \ldots \ldots$

$2.1 \phi_{k}^{*} \geq f\left(x^{k}\right) \ldots \ldots \ldots \ldots \ldots \ldots \ldots$

2.2 Sequência de funções aproximadoras . . . . . . . . . . . . . . . . 17

2.3 Construção de $\left\{\phi_{k}(\cdot)\right\} \ldots \ldots \ldots$. . . . . . . . . . . . . 19

$2.4 y^{k}$ a partir de $x^{k}$ na direção $\left(v^{k}-x^{k}\right) \ldots \ldots \ldots . \ldots \ldots 23$

$2.5 x^{k+1}$ na direção $-\nabla f\left(y^{k}\right) \ldots \ldots \ldots \ldots . \ldots . \ldots . \ldots . \ldots 24$

$2.6 \quad v^{k+1}$ na direção $-\nabla f\left(y^{k}\right) \ldots \ldots \ldots . \ldots . \ldots . \ldots . \ldots . \ldots 24$

3.1 Distância de Bregman de $x$ em relação a $y \quad \ldots . . . . . .37$

3.2 Escolha de $y^{k} \ldots \ldots \ldots$. . . . . . . . . . . . . . . . . . 43

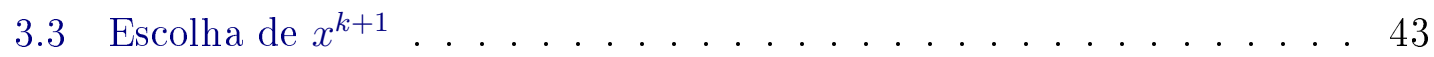

$3.4 f(z) \leq f\left(x^{k}\right) \ldots \ldots \ldots \ldots \ldots \ldots \ldots$

$3.5 \theta=\beta+\alpha_{A T}(1-\beta) \ldots \ldots \ldots \ldots \ldots$. . . . . . . . . . 54

$3.6 \hat{x}=y^{k}+\alpha_{A T}\left(v^{k+1}-v^{k}\right) \ldots \ldots \ldots \ldots \ldots \ldots \ldots$

3.7 Aproximação superior . . . . . . . . . . . . . . . 55

4.1 (PassoAT $-\mathrm{KL}) \times($ PassoAT $-\mathrm{E}) \ldots \ldots \ldots 68$

4.2 Comportamento do método Auslender e Teboulle . . . . . . . . . . . 69

$4.3($ PassoAT $-\mathrm{E}) \times(\mathrm{xLy}) \ldots \ldots \ldots . \ldots . \ldots 71$

$4.4(\mathrm{xLy}) \times(\mathrm{xLx}) \ldots \ldots \ldots \ldots 72$

$4.5 \quad(\mathrm{xLy}) \times(\mathrm{xLy}-2) \ldots \ldots \ldots 73$

$4.6(\mathrm{xLy}) \times(\mathrm{xLy}-\mathrm{yb}) \ldots \ldots \ldots \ldots . \ldots \ldots 74$

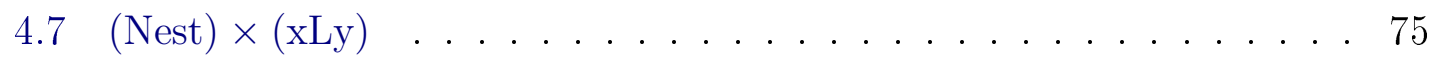

4.8 Métodos com projeção em relação a $y^{k}$ e passo $\xi_{s p g} \quad \ldots . . .77$

$4.9 \quad(\mathrm{xSPGy}) \times(\mathrm{xSPGyb}) \ldots \ldots \ldots . \ldots . \ldots 78$

4.10 Métodos com projeção em relação a $x^{k}$ e passo $\xi_{s p g}$. . . . . . . . . . 80

$4.11(\mathrm{xSPGxb}) \times(\mathrm{xSPGxb}-2) \ldots \ldots . \ldots . \ldots 81$ 
$4.12(\mathrm{xSPGyb}) \times(\mathrm{xSPGxb}-2) \ldots \ldots \ldots . \ldots . \ldots . \ldots 8$

$4.13(\mathrm{xSPGxb}-2) \times(\mathrm{xSPGxb}-\mathrm{yb}-2) \ldots \ldots . \ldots . \ldots 83$

4.14 (Nest) $\times(\mathrm{xSPGxb}-2) \ldots \ldots \ldots . \ldots . \ldots . \ldots . \ldots$

$4.15(\mathrm{xLy}) \times(\mathrm{xSPGxb}-2) \ldots \ldots \ldots \ldots$ 


\section{Introdução}

Neste trabalho estudamos métodos ótimos para minimização de funções convexas diferenciáveis restritas a conjuntos convexos sobre os quais é simples de projetar.

Estes esquemas de resolução de problemas foram introduzidos pelo matemático russo Yurii Nesterov que iniciou seu estudo nesta área durante seu doutoramento e os primeiros resultados foram publicados no artigo [13] no ano de 1983. Mais tarde, no ano de 2004, Nesterov publicou o livro entitulado Introductory Lectures on Convex Optimization onde detalhou todo seu trabalho sobre métodos ótimos em otimização convexa.

Os resultados mais importantes dos trabalhos citados acima, e que serviram de motivação para nosso estudo, estabelecem limites para o número de iterações que um método baseado apenas em gradientes necessita para atingir uma solução aproximada para um problema de otimização convexa. Nesterov desenvolveu algoritmos que usam apenas esse número mínimo de iterações, denominados de métodos ótimos.

Uma das desvantagens destes métodos é o fato de usarem explicitamente a constante de Lipschitz $L$ do gradiente da função objetivo e o parâmetro de convexidade forte $\mu$ da função objetivo. Em 2007 no artigo [15], Nesterov reescreve seu método mostrando que é possível trabalhar com uma aproximação da constante de Lipschitz, que pode ser estimada de forma adaptativa, sem perder a otimalidade.

Em [7] Gonzaga e Karas modificam o método de Nesterov para problemas sem restrições. Neste trabalho, os autores apresentam um esquema que também não usa a constante de Lipschitz do gradiente da função objetivo, desenvolvem uma forma adaptativa para estimar o parâmetro de convexidade forte $\mu$ e melhoram as escolhas dos passos do algoritmo, fazendo buscas lineares do tipo Wolfe e Armijo para que em cada iteração seja possível obter um maior decréscimo no valor da função objetivo. Um dos pontos altos deste trabalho se encontra na interpretação geométrica do método. 
No início do ano de 2010 encontramos um trabalho de Auslender e Teboulle que contém uma extensão do método de Nesterov sem restrições para o problema com restrições [2]. Para captar as informações das restrições eles usam como ferramenta distâncias de Bregman definidas no interior do conjunto viável, com a propriedade de coercividade. Uma das desvantagens deste método é que ele não pode usar a distância euclidiana, além disto, o algoritmo usa explicitamente a constante de Lipschitz do gradiente da função objetivo. Este trabalho foi nossa maior fonte de inspiração para melhorar a eficiência de métodos ótimos para problemas restritos.

Neste trabalho conseguimos reescrever o método de Auslender e Teboulle usando distância euclidiana, uma ferramenta mais simples e mais intuitiva que distâncias de Bregman. De forma similar ao que Nesterov fez em [15], desenvolvemos um critério para estimar a constante de Lipschitz. Além disto, inspirados no trabalho de Gonzaga e Karas, mostramos que é possível melhorar a escolha dos pontos obtendo um decréscimo maior no valor da função objetivo, o que resulta em métodos mais eficientes.

O texto foi estruturado da seguinte maneira:

- Capítulo 1: apresentamos os conceitos e resultados básicos de otimização convexa;

- Capítulo 2: descrevemos o método ótimo de Nesterov para otimização de funções convexas sem restrições;

- Capítulo 3: reescrevemos os métodos ótimos de Nesterov e Auslender e Teboulle para o caso restrito. Em seguida propomos um novo método ótimo que permite a escolha de novos pontos de forma a melhorar a eficiência;

- Capítulo 4: neste capítulo exibimos todos os testes numéricos que foram feitos para decidir qual é a melhor versão do nosso método e em seguida comparamos com o método ótimo de Nesterov. 


\section{Capítulo 1}

\section{Conceitos Básicos}

Neste capítulo apresentamos uma série de definições e resultados referentes à Análise Convexa e Otimização que são básicos para nosso trabalho. Para tanto, usamos como base os livros Convex Analysis and Optimization [3] de Dimitri Bertsekas, Introductory Lectures on Convex Optimization [14] de Yurii Nesterov e Otimização - Volume 1 [11] de Alexey Izmailov e Mikhail Solodov e a dissertação de mestrado de Paulo J. S. Silva intitulada Método de Ponto Proximal e Separadores [16].

O conceito de convexidade é muito importante na área de otimização, principalmente pelo fato que, sob hipóteses de convexidade, as condições necessárias de otimalidade passam a ser condições suficientes. Em particular qualquer solução local é solução global. Além disso, no caso de otimização convexa é possível desenvolver a teoria de dualidade, ou seja, associar ao problema original um outro problema que é equivalente ao original e muitas vezes mais fácil de resolver, [11, 14].

Definição 1.1. Um subconjunto $C \subset \mathbb{R}^{n}$ é dito convexo se, para qualquer $\alpha \in[0,1]$ e quaisquer pontos $x, y \in C$, o ponto definido por $(1-\alpha) x+\alpha y$ pertence a $C$.

Isso quer dizer que um conjunto é convexo quando, para quaisquer dois pontos deste conjunto, o segmento que une estes pontos está inteiramente contido nele. Apresentamos abaixo uma ilustração deste fato. 


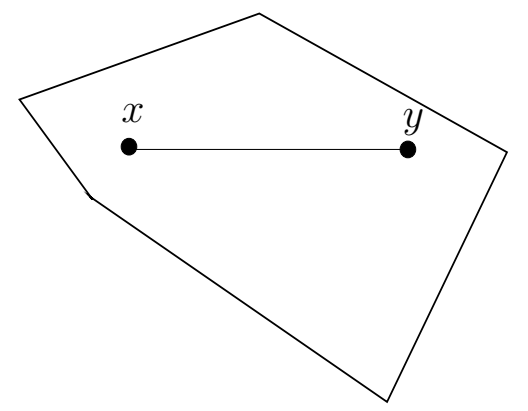

(a) Convexo

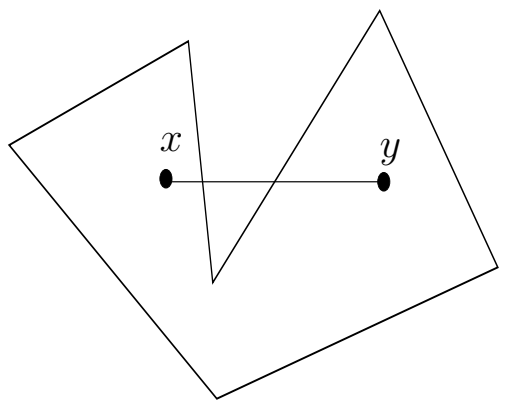

(b) Não convexo

Figura 1.1: Conjunto Convexo

O conjunto vazio, o espaço $\mathbb{R}^{n}$ e um conjunto que contém um único ponto, são trivialmente conjuntos convexos.

Definição 1.2. Seja $C \subset \mathbb{R}^{n}$ um conjunto convexo. Dizemos que uma função $f$ : $C \rightarrow \mathbb{R}$ é convexa em $C$ quando, para todo $x, y \in C$ e todo $\alpha \in[0,1]$, valer

$$
f(\alpha x+(1-\alpha) y) \leq \alpha f(x)+(1-\alpha) f(y)
$$

Se a desigualdade for estrita, então $f(\cdot)$ é dita estritamente convexa em C. Se, para quaisquer pontos $x$ e $y \in C$ e $\alpha \in[0,1]$, valer a desigualdade

$$
f(\alpha x+(1-\alpha) y) \leq \alpha f(x)+(1-\alpha) f(y)-\frac{1}{2} \mu \alpha(1-\alpha)\|x-y\|^{2} .
$$

dizemos que $f(\cdot)$ é fortemente convexa em $C$ com parâmetro de convexidade $\mu>$ 0 .

A próxima figura ilustra o comportamento de uma função convexa:

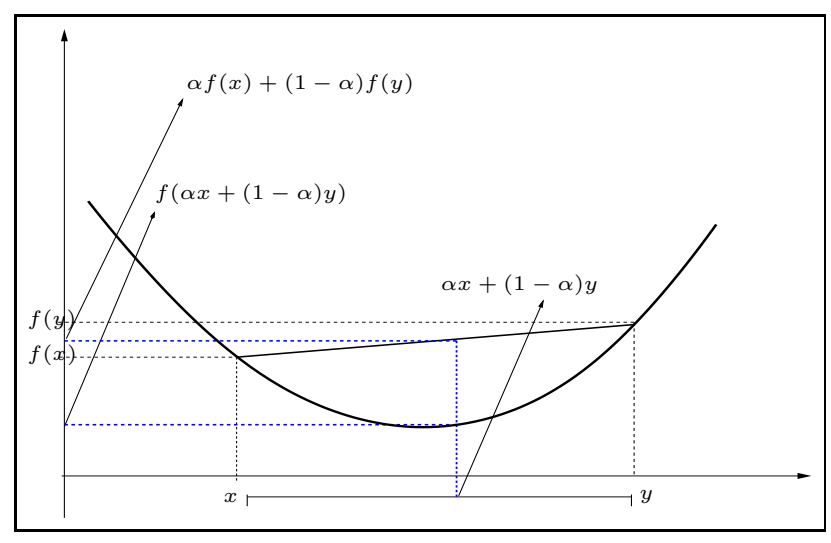

Figura 1.2: Função Convexa 
Dessa forma, dados dois pontos no domínio da função, a imagem de uma combinação convexa destes dois pontos está sempre abaixo da combinação convexa das imagens destes dois pontos.

Consideramos agora, uma extensão da função $f(\cdot)$ que pode assumir valores $-\infty$ ou $\infty$. Para isto extendemos o domínio da função, ou seja, consideramos um subconjunto $S \subset \mathbb{R}^{n}$, tal que $C \subset S$ e definimos $f: S \rightarrow[-\infty, \infty]$.

Definição 1.3. Seja $f: S \rightarrow[-\infty, \infty]$. Chamamos de conjunto de nivel de $f(\cdot)$ o conjunto $\mathcal{L}_{f}(r)=\left\{x \in \mathbb{R}^{n} \mid f(x) \leq r\right\}$.

Definição 1.4. Seja $f: S \rightarrow[-\infty, \infty]$. O dominio efetivo de $f(\cdot)$ é dado pelo conjunto dom $f=\{x \in S \mid f(x)<\infty\}$.

Definição 1.5. Seja $f: S \rightarrow[-\infty, \infty]$. O conjunto epi $f=\left\{(x, r) \in \mathbb{R}^{n} \times \mathbb{R} \mid f(x) \leq\right.$ $r\}$ é chamado de epígrafe de $f(\cdot)$.

Definição 1.6. Seja $C$ um subconjunto convexo de $\mathbb{R}^{n}$. Uma função a valores extendidos $f: C \rightarrow[-\infty, \infty]$ é uma função convexa se sua epígrafe é um subconjunto convexo de $\mathbb{R}^{n+1}$.

Agora definimos função semi-contínua inferior e função própria. Estas definições serão úteis no terceiro capítulo onde mostramos o método ótimo de Auslender e Teboulle que utilizam distâncias de Bregman.

Definição 1.7. Seja $S \subset \mathbb{R}^{n}$. Uma função $f: S \rightarrow[-\infty, \infty]$ é semi-contínua inferior (sci) em um ponto $\bar{x} \in S$ se, para toda sequência $\left\{x^{k}\right\} \subset S$ que converge para $\bar{x}$ é possivel verificar

$$
f(\bar{x}) \leq \liminf _{k \rightarrow \infty} f\left(x^{k}\right)
$$

Definição 1.8. Seja $S \subset \mathbb{R}^{n}$. Uma função $f: S \rightarrow[-\infty, \infty]$ é própria em $S$ se, para pelo menos um $x \in S$ acontecer $f(x)<\infty$ e $f(x)>\infty$ para todo $x \in S$.

A próxima definição refere-se a função indicadora de um determinado conjunto. Esta função é útil quando queremos transformar o problema de otimizar uma função em um conjunto convexo em um problema irrestrito. Fazendo este tipo de transformação, Nesterov desenvolveu um método ótimo para otimizar uma função convexa restrita a um conjunto convexo [15].

Definição 1.9. Seja um subconjunto $S \subset \mathbb{R}^{n}$ não vazio. Uma função $I_{S}: \mathbb{R}^{n} \rightarrow$ $(-\infty, \infty]$ dada por 


$$
I_{S}(x)=\left\{\begin{array}{l}
0, \text { se } x \in S \\
+\infty, \text { caso contrário }
\end{array}\right.
$$

é denominada função indicadora do conjunto $S$.

Apresentamos agora uma proposição cuja demonstração pode ser encontrada em [3].

Proposição 1.10. Seja $I_{C}(\cdot)$ a função indicadora do conjunto $C \subset \mathbb{R}^{n}$. Então $I_{C}(\cdot)$ é uma função convexa se, e somente se, $C$ é um conjunto convexo do $\mathbb{R}^{n}$.

\subsection{Funções convexas continuamente diferenciáveis}

Nesta seção apresentamos uma série de resultados envolvendo funções convexas que possuem também a propriedade de serem diferenciáveis.

Teorema 1.11. Seja um conjunto convexo aberto $C \subset \mathbb{R}^{n}$ e uma função diferenciável $f: C \rightarrow \mathbb{R} . f(\cdot)$ é convexa em $C$ se, e somente se, para quaisquer $x, y \in C$ tem-se

$$
f(x) \geq f(y)+\langle\nabla f(y), x-y\rangle .
$$

A função $f(\cdot)$ é estritamente convexa em $C$ se, e somente se, para quaisquer dois pontos distintos $x$ e $y \in C$, a desigualdade (1.2) for estrita. E, $f(\cdot)$ é fortemente convexa em $C$, com parâmetro de convexidade $\mu>0$ se, e somente se, para quaisquer dois pontos $x$ e $y \in C$ valer

$$
f(x) \geq f(y)+\langle\nabla f(y), x-y\rangle+\frac{\mu}{2}\|x-y\|^{2} .
$$

A demonstração deste teorema pode ser vista em [3, 14]. Abaixo, ilustramos uma função convexa diferenciável. Neste caso, uma aproximação linear dada pelo gradiente, em qualquer ponto do domínio da função, é sempre uma aproximação inferior para a função convexa. 


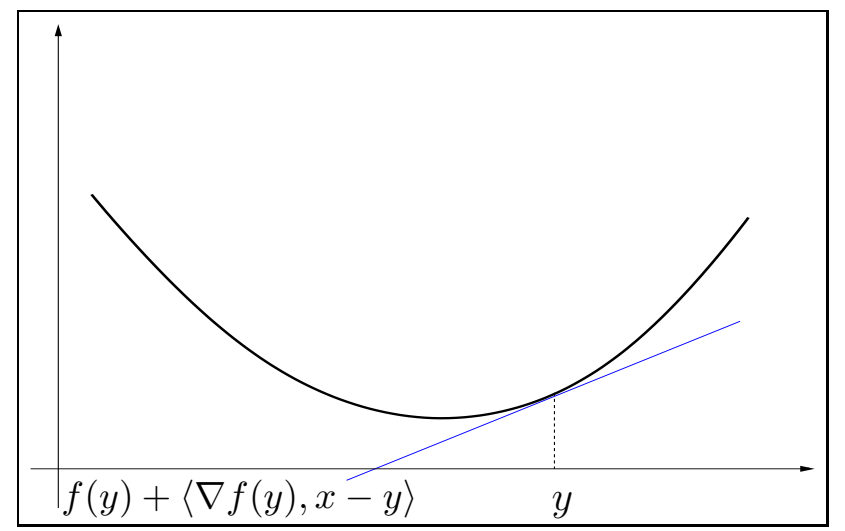

Figura 1.3: Função Convexa Diferenciável

Definição 1.12. Seja $S \subset \mathbb{R}^{n}$ um subconjunto aberto e uma função $f: S \rightarrow \mathbb{R}$ continuamente diferenciável. Dizemos que o gradiente de $f(\cdot)$ é Lipschitz contínuo em $S$, se existir um escalar $L>0$ tal que

$$
\|\nabla f(x)-\nabla f(y)\| \leq L\|x-y\|
$$

para todo $x, y \in S$.

Proposição 1.13. Seja $C \subset \mathbb{R}^{n}$ um conjunto convexo e aberto e $f: C \rightarrow \mathbb{R}$ uma função convexa em $C$, diferenciável com gradiente Lipschitz continuo, com constante de Lipschitz $L>0$, em C. Então para todo $x, y \in S$ temos

$$
f(x) \leq f(y)+\langle\nabla f(y), x-y\rangle+\frac{L}{2}\|x-y\|^{2} .
$$

Demonstração:

Para todo $x, y \in C$, podemos escrever a seguinte igualdade

$$
\begin{aligned}
f(x) & =f(y)+\int_{0}^{1}\langle\nabla f(y+\tau(x-y)), x-y\rangle d \tau \\
& =f(y)+\langle\nabla f(y), x-y\rangle+\int_{0}^{1}\langle\nabla f(y+\tau(x-y))-\nabla f(y), x-y\rangle d \tau .
\end{aligned}
$$


Assim,

$$
\begin{aligned}
f(x)-f(y)-\langle\nabla f(y), x-y\rangle & =\left|\int_{0}^{1}\langle\nabla f(y+\tau(x-y))-\nabla f(y), x-y\rangle d \tau\right| \\
& \leq \int_{0}^{1}|\langle\nabla f(y+\tau(x-y))-\nabla f(y), x-y\rangle| d \tau \\
& \leq \int_{0}^{1} \|\langle\nabla f(y+\tau(x-y))-\nabla f(y)\|\| x-y \| d \tau \\
& \leq \int_{0}^{1} \tau L\|x-y\|^{2} d \tau=\frac{L}{2}\|x-y\|^{2} .
\end{aligned}
$$

Enunciaremos agora três teoremas cujas demonstrações podem ser vistas em $[3,14]$.

Teorema 1.14. Seja $f: S \rightarrow \mathbb{R}$ duas vezes continuamente diferenciável em um conjunto aberto $S \subset \mathbb{R}^{n}$. Então, $f(\cdot)$ é convexa em $S$ se, e somente se, para todo $x \in S$,

$$
\nabla^{2} f(x) \succcurlyeq 0
$$

Ou seja, a matriz Hessiana associada a função $f(\cdot)$ é semidefinida positiva.

Teorema 1.15. Seja $f: S \rightarrow \mathbb{R}$, duas vezes continuamente diferenciável em um conjunto aberto $S \subset \mathbb{R}^{n}$. A função $f(\cdot)$ é convexa em $S$ e possui gradiente Lipschitz continuo, com constante $L>0$, em $S$ se, e somente se, para todo $x \in S$,

$$
0 \preceq \nabla^{2} f(x) \preceq L I_{n}
$$

Teorema 1.16. Seja $f: S \rightarrow \mathbb{R}$, duas vezes continuamente diferenciável em um conjunto aberto $S \subset \mathbb{R}^{n}$. A função $f(\cdot)$ é fortemente convexa, com parâmetro $\mu>0$, em $S$ se, e somente se, para todo $x \in S$,

$$
\nabla^{2} f(x) \succcurlyeq \mu I_{n}
$$

Dos dois últimos teoremas temos que,

$$
\mu I_{n} \preceq \nabla^{2} f(x) \preceq L I_{n}
$$

de onde podemos concluir que uma função $f(\cdot)$ convexa e duas vezes diferenciável está limitada acima por uma aproximação fortemente convexa, cujo parâmetro de 
convexidade é o valor da constante de Lispchitz, $L>0$, e esta está limitada abaixo por uma aproximação fortemente convexa, cujo parâmetro de convexidade é o valor do parâmetro de convexidade forte da própria função $f(\cdot)$.

Quando uma função não possui a propriedade de ser diferenciável em algum ponto do seu domínio, não podemos falar em gradiente da função neste ponto. Por exemplo, em $\mathbb{R}$ a função módulo não é diferenciável no ponto 0 , pois neste ponto é possível definir infinitas aproximaçõs lineares. Este fato é ilustrado na figura 1.4.

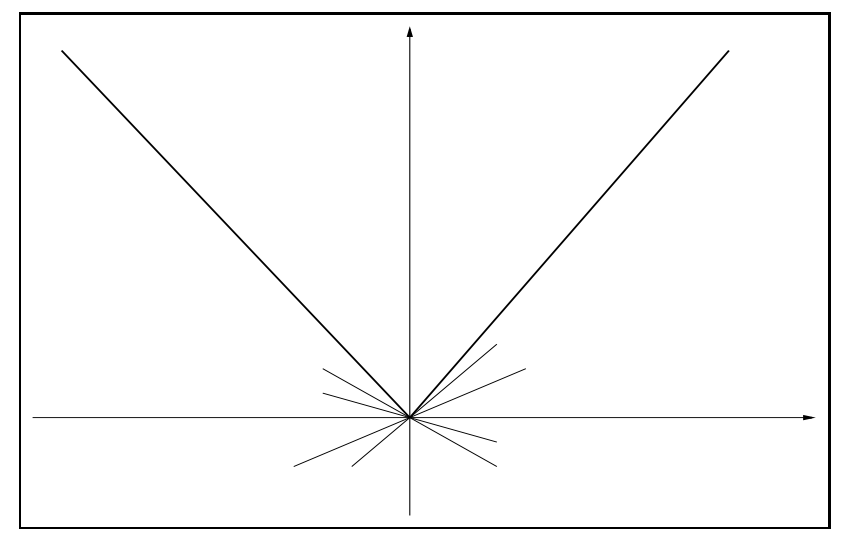

Figura 1.4: $f(x)=|x|$

Porém, existe uma concepção que generaliza o conceito de gradiente e que é aplicado a funções onde em um determinando ponto é possível construir aproximações inferiores. Na próxima definição apresentamos este conceito.

Definição 1.17. Seja $C \subset \mathbb{R}^{n}$ um conjunto convexo e uma função convexa $f$ : $C \rightarrow \mathbb{R}$. O subdiferencial, representado por $\partial f(x)$, de $f(\cdot)$ no ponto $x \in \operatorname{dom} f$ é definido pelo seguinte conjunto:

$$
\partial f(x)=\left\{g \in \mathbb{R}^{n} \mid f(y) \geq f(x)+\langle g, y-x\rangle \text { para todo } y \in C\right\} .
$$

Um vetor $g \in \partial f(x)$ é chamado subgradiente de $f$ em $x$.

Esse conceito estende o definição de gradiente. Em particular, se a função é diferenciável em todos os pontos, então o subdiferencial associado a cada ponto é um conjunto unitário formado pelo gradiente da função neste ponto. O entendimento de subgradiente será útil no terceiro capítulo, onde apresentaremos o método ótimo desenvolvido por Nesterov para problemas com restrições. 


\subsection{Condições de Otimalidade}

Nessa seção escrevemos as condições para que um ponto seja uma solução de um problema de otimização convexa.

Considere, inicialmente, o problema de otimização de uma função convexa sem restrições, ou seja,

$$
\underset{x \in \mathbb{R}^{n}}{\operatorname{minimizar}} f(x)
$$

onde $f: \mathbb{R}^{n} \longrightarrow \mathbb{R}$ é convexa e diferenciável em $\mathbb{R}^{n}$.

Uma condição necessária para que um ponto $x^{*}$ seja solução de um determinando problema de otimização é que o gradiente da função neste ponto seja nulo. No próximo teorema veremos que sob hipótese de convexidade, esta condição é também suficiente.

Teorema 1.18. Seja $f: \mathbb{R}^{n} \rightarrow \mathbb{R}$ convexa e diferenciável em $\mathbb{R}^{n}$. Se o gradiente de $f(\cdot)$ em $x^{*}$ for nulo, isto é $\nabla f\left(x^{*}\right)=0$, então o ponto $x^{*}$ é solução global do problema (1.3).

Demonstração:

Como $f(\cdot)$ é uma função convexa diferenciável, segue da desigualdade (1.2), que para todo $x \in \mathbb{R}$,

$$
f(x) \geq f\left(x^{*}\right)+\left\langle\nabla f\left(x^{*}\right), x-x^{*}\right\rangle .
$$

Mas, usando a hipótese que $\nabla f\left(x^{*}\right)=0$, temos que para todo $x \in \mathbb{R}$,

$$
f(x) \geq f\left(x^{*}\right) .
$$

Ou seja, $x^{*}$ é um minimizador irrestrito de $f(\cdot)$.

Quando $f(\cdot)$ não é diferenciável podemos enunciar um teorema análogo ao teorema anterior, apenas trocando o gradiente pelo subgradiente.

Então, quando construimos métodos para resolver o problema 1.3, devemos procurar pontos que satisfaçam a condição de anular o gradiente da função objetivo. 
Considere agora o problema de otimização de uma função convexa em um conjunto convexo, isto é,

$$
\begin{aligned}
& \underset{x \in C}{\operatorname{minimizar}} f(x) \\
&
\end{aligned}
$$

em que $C$ é um conjunto não vazio, convexo e fechado em $\mathbb{R}^{n}$ e $f: \mathbb{R}^{n} \rightarrow \mathbb{R}$ é uma função convexa e diferenciável em $C$.

O próximo teorema fornece uma condição necessária e suficiente para que um ponto seja solução ótima do problema (1.4). Para a demonstração deste teorema precisamos definir o que é uma direção de descida.

Definição 1.19. Seja $f: \mathbb{R}^{n} \rightarrow \mathbb{R}$ uma função convexa e $\bar{x}$ um ponto de $\mathbb{R}^{n}$. Uma direção $d \in \mathbb{R}^{n}$ é dita uma direção de descida de $f(\cdot)$ em $\bar{x}$ se:

$$
\langle\nabla f(\bar{x}), d\rangle<0
$$

Isto quer dizer que a derivada direcional de $f(\cdot)$, no ponto $\bar{x}$ na direção d, é negativa.

Teorema 1.20. Seja $C$ um conjunto convexo e fechado do $\mathbb{R}^{n}$ e $f: C \rightarrow \mathbb{R}$ uma função convexa e diferenciável em $C$. Um ponto $x^{*}$ é uma solução do problema 1.4 se e somente se, para todo $x \in C$,

$$
\left\langle\nabla f\left(x^{*}\right), x-x^{*}\right\rangle \geq 0
$$

Demonstração:

Assumimos que $x^{*}$ é uma solução do problema (1.4) e que existe algum $x \in C$ tal que

$$
\left\langle\nabla f\left(x^{*}\right), x-x^{*}\right\rangle<0 .
$$

Esta última desigualdade nos diz que $x-x^{*}$ é uma direção de descida. Considere então, a função $\psi(\alpha)=f\left(x^{*}+\alpha\left(x-x^{*}\right)\right)$, com $\alpha \in[0,1]$. Note que o ponto $x^{*}+$ $\alpha\left(x-x^{*}\right)$ é viável para $\alpha$ suficientemente pequeno, pois é uma combinação convexa entre $x$ e $x^{*}$. Observe ainda, que

$$
\psi(0)=f\left(x^{*}\right), \psi^{\prime}(0)=\left\langle\nabla f\left(x^{*}\right), x-x^{*}\right\rangle .
$$

Assim, para $\alpha$ suficientemente pequeno temos

$$
f\left(x^{*}+\alpha\left(x-x^{*}\right)\right)=\psi(\alpha)<\psi(0)=f\left(x^{*}\right) .
$$


O que contradiz o fato de $x^{*}$ ser solução do problema (1.4). Assim, provamos a primeira parte do teorema. Por outro lado, como $f(\cdot)$ é uma função convexa e diferenciável, temos que para todo $x \in C$,

$$
f(x) \geq f\left(x^{*}\right)+\left\langle\nabla f\left(x^{*}\right), x-x^{*}\right\rangle .
$$

Mas por hipótese, o ponto $x^{*}$ satisfaz a desigualdade (1.5). Então, para todo $x \in C$,

$$
f(x) \geq f\left(x^{*}\right) .
$$

Ou seja, $x^{*}$ é solução do problema (1.4). E portanto, provamos a segunda parte do teorema.

\subsection{Otimalidade de um método}

Essa seção é dedicada a apresentação do significado de otimalidade de um método de primeira ordem para uma classe de funções $S$. Este conceito refere-se ao esforço computacional necessário para encontrar um minimizador de uma função $f(\cdot)$ em um conjunto $S$. Na próxima definição apresentamos o significado de resolver um problema de otimização segundo Nesterov [14].

Definição 1.21. Seja $f^{*}$ o valor ótimo de um problema de otimização (1.4). Dado $\epsilon>0$, resolver este problema, com precisão $\epsilon>0$, significa encontrar uma solução aproximada $\bar{x} \in C$ de forma que $f(\bar{x})-f^{*}<\epsilon$.

Após desenvolver um método de otimização a pergunta que surge, naturalmente, é quão eficiente é este método em relação a uma classe de problemas. Ou seja, se é possível prever o esforço computacional gasto por este método para resolver todos os problemas. Por outro lado, considerando uma classe de problemas, é também interessante questionar sobre a possibilidade de se estimar o menor esforço computacional possível que uma classe de métodos pode fazer para resolvê-los. É neste contexto que surgem os conceitos de cota de complexidade inferior e cota de complexidade superior.

Considerando uma função positiva $g: \mathbb{R}^{n} \rightarrow \mathbb{R}$. Dizemos que a cota de complexidade inferior de uma classe $\mathcal{F}$ de funções e uma classe de métodos de otimização $\mathcal{M}$ é $\Omega(g(\epsilon))$ quando para todo método $M$ da classe de métodos $\mathcal{M}$, 
existe uma função $\bar{f} \in \mathcal{F}$ para qual o método gastará $\Omega(g(\epsilon))$ iterações com precisão $\epsilon$. Isto quer dizer que, existe $N_{1}>0$ que depende das classes $\mathcal{M}$ e $\mathcal{F}$ tal que se $\left\{x^{k}\right\}$ é a sequência gerada pelo método $M$ de $\mathcal{M}$ e se $k \leq N_{1} g(\epsilon)$, então

$$
\bar{f}\left(x^{k}\right)-\bar{f}^{*} \geq \epsilon .
$$

A complexidade inferior está relacionada com as funções de $\mathcal{F}$ que são difíceis de minimizar pelos métodos de $\mathcal{M}$.

Por outro lado, considere uma função positiva $h: \mathbb{R}^{n} \rightarrow \mathbb{R}$. Fixado um método $M$, a cota de complexidade superior deste método é $O(h(\epsilon))$, quando ele minimiza, com precisão $\epsilon$, toda função da classe $\mathcal{F}$ em $O(h(\epsilon))$ iterações. Isto quer dizer que, existe $N_{2}>0$ que depende do método $M$ tal que se $\left\{x^{k}\right\}$ é a sequência gerada pelo método $M$ para minimizar $f \in \mathcal{F}$ e $k \geq N_{2} h(\epsilon)$, então

$$
f\left(x^{k}\right)-f^{*} \leq \epsilon
$$

Definição 1.22. Um método $M$, de uma classe de métodos $\mathcal{M}$, é ótimo para uma classe $\mathcal{F}$ de funções quando a sua cota de complexidade superior é proporcional a cota de complexidade inferior da classe de funções $\mathcal{F}$ e da classe de métodos $\mathcal{M}$.

Nesse trabalho estamos interessados em métodos ótimos de primeira ordem (avaliam apenas valor da função e valor do gradiente em um ponto) para a classe de funções convexas. Nesterov mostrou, em [14], que existe uma função $\bar{f}$ na classe de funções convexas tal que para todo método de primeira ordem,

$$
\bar{f}\left(x^{k}\right)-\bar{f}^{*} \geq \frac{3 L\left\|x^{0}-x^{*}\right\|^{2}}{32(k+1)^{2}} .
$$

Isto quer dizer que:

$$
\bar{f}\left(x^{k}\right)-\bar{f}^{*}=\Omega\left(\frac{1}{k^{2}}\right)
$$

De onde concluímos que a cota de complexidade inferior para a classe de funções convexas e os métodos de primeira ordem é $\Omega\left(\frac{1}{\sqrt{\epsilon}}\right)$.

Dessa forma, para um método de primeira ordem ser considerado ótimo, para a classe de funções convexas, ele deve satisfazer a cota de complexidade superior $O\left(\frac{1}{\sqrt{\epsilon}}\right)$. Porém, para isto, basta que: 


$$
f\left(x^{k}\right)-f^{*}=O\left(\frac{1}{k^{2}}\right)
$$

Em resumo, podemos dizer que um método de primeira ordem é ótimo para a classe de funções convexas quando determina um solução, com precisão $\epsilon$, em $\frac{1}{\sqrt{\epsilon}}$ iterações.

No próximo capítulo apresentamos um método ótimo para otimização de uma função convexa sem restrições. 


\section{Capítulo 2}

\section{Um método ótimo para problemas sem restrições}

Considere o problema de otimização convexa irrestrita

$$
\underset{x \in \mathbb{R}^{n}}{\operatorname{minimizar}} f(x)
$$

onde $f: \mathbb{R}^{n} \longrightarrow \mathbb{R}$ é convexa e continuamente diferenciável em $\mathbb{R}^{n}$, e o gradiente da função objetivo é Lipschitz contínuo, com constante de Lipschitz $L>0$, ou seja, para todo $x, y \in \mathbb{R}^{n}$

$$
\|\nabla f(x)-\nabla f(y)\| \leq L\|x-y\|
$$

Nas próximas páginas desse capítulo apresentamos o método ótimo de Nesterov para otimização de uma função convexa sem restrições, descrito no segundo capítulo do livro Introductory lectures on convex optimization [15]. A importância desse método está no fato de servir de base para métodos para problemas com restrições que vamos desenvolver e melhorar no próximo capítulo.

\subsection{Ideia do método de Nesterov}

Conforme foi exposto no capítulo anterior, para que um método de primeira ordem seja ótimo para a classe de funções convexas ele deve satisfazer: 


$$
f\left(x^{k}\right)-f^{*}=O\left(\frac{1}{k^{2}}\right)
$$

Para desenvolver um método ótimo, Nesterov construiu uma sequência de funções $\left\{\phi_{k}(\cdot)\right\}$ que aproximam a função objetivo $f(\cdot)$ de modo que seja possível escrever a seguinte desigualdade:

$$
f\left(x^{k}\right)-f\left(x^{*}\right) \leq \lambda_{k}\left(\phi_{0}\left(x^{*}\right)-f\left(x^{*}\right)\right)
$$

onde $\lambda_{k} \geq 0$ e $\phi_{0}(\cdot)$ é a primeira função da sequência $\left\{\phi_{k}(\cdot)\right\}$. Desta forma, para que o método seja ótimo, é preciso que $\lambda_{k}=O\left(\frac{1}{k^{2}}\right)$.

Para conseguir escrever a desigualdade (2.3), a sequência de funções $\left\{\phi_{k}(\cdot)\right\}$ que aproximam a função $f(\cdot)$ deve ser construída de forma que em cada iteração $k \geq 0$ e $\alpha_{k} \in(0,1)$ a seguinte razão seja satisfeita, para todo $x \in \mathbb{R}^{n}$,

$$
\phi_{k+1}(x)-f(x) \leq\left(1-\alpha_{k}\right)\left(\phi_{k}(x)-f(x)\right) .
$$

É imediato observar que desta desigualdade decorre que:

$$
\phi_{k}(x)-f(x) \leq\left(1-\alpha_{k-1}\right)\left(1-\alpha_{k-2}\right) \cdots\left(1-\alpha_{0}\right)\left(\phi_{0}(x)-f(x)\right) .
$$

Além disso, a sequência $\left\{x^{k}\right\}$ deve ser determinada de forma que, para todo $k \geq 0$, o valor mínimo de $\phi_{k}(\cdot)$, que chamamos de $\phi_{k}^{*}$, seja maior ou igual ao valor de $f\left(x^{k}\right)$. A condição é mostrada na figura abaixo.

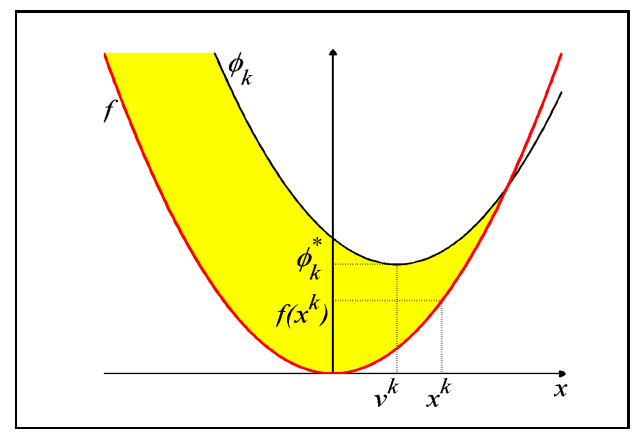

Figura 2.1: $\phi_{k}^{*} \geq f\left(x^{k}\right)$

Observe que se a sequência $\left\{\phi_{k}(\cdot)\right\}$ satisfaz a desigualdade (2.5) e do fato que $\phi_{k}^{*} \geq$ $f\left(x^{k}\right)$, para todo $k \geq 0$, segue facilmente $(2.3)$ com 


$$
\lambda_{k}:=\prod_{i=0}^{k-1}\left(1-\alpha_{i}\right) .
$$

Para prosseguir com a ideia da construção da sequência $\left\{\phi_{k}(\cdot)\right\}$ definimos o conceito de função simples.

Definição 2.1. Seja $\phi_{k}: \mathbb{R}^{n} \rightarrow \mathbb{R}$ uma função fortemente convexa. Dizemos que $\phi_{k}(\cdot)$ é função simples se é da forma:

$$
\phi_{k}(x)=\phi_{k}^{*}+\frac{\gamma_{k}}{2} D\left(x, v^{k}\right)
$$

onde $\phi_{k}^{*} \in \mathbb{R}$ é o valor ótimo de $\phi_{k}(\cdot), v^{k} \in \mathbb{R}^{n}$ é o minimizador irrestrito de $\phi_{k}(\cdot)$, $\gamma_{k} \in \mathbb{R}$ é o parâmetro de convexidade forte e $D\left(x, v^{k}\right)$ é uma função que representa uma distância em relação a $v^{k}$. Além disso, se $D\left(x, v^{k}\right)=\left\|x-v^{k}\right\|^{2}$ dizemos que $\phi(\cdot)$ é função quadrática convexa simples.

No decorrer deste capítulo mostramos que as funções da sequência $\left\{\phi_{k}(\cdot)\right\}$ são funções quadráticas convexas simples. E na figura abaixo mostramos uma sequência de funções quadráticas convexas simples se aproximando da função $f(\cdot)$ (que está por baixo).

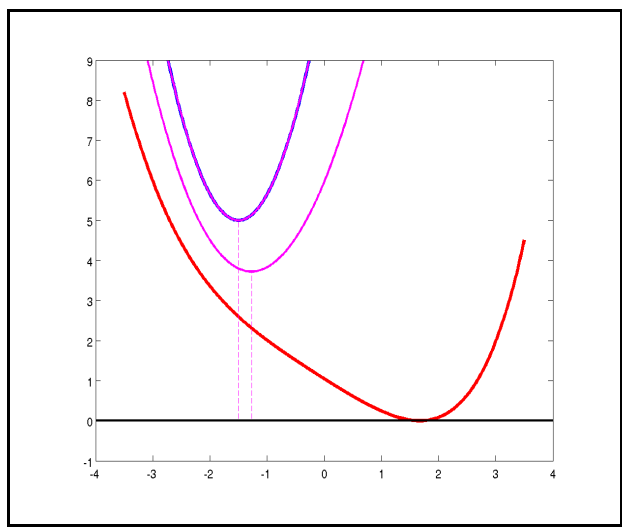

Figura 2.2: Sequência de funções aproximadoras

Para justificar a construção da sequência $\left\{\phi_{k}(\cdot)\right\}$, satisfazendo (2.4) e (2.3), Nesterov introduziu o conceito de sequência de estimativas.

Definição 2.2. Um par de sequências $\left\{\phi_{k}(\cdot)\right\}$ e $\left\{\lambda_{k}\right\}, \lambda_{k} \geq 0$, é chamado uma 
sequência de estimativas da função $f(\cdot)$ se

$$
\lambda_{k} \rightarrow 0
$$

e para qualquer $x \in \mathbb{R}^{n}$ e todo $k \geq 0$ temos

$$
\phi_{k}(x)-f(x) \leq \lambda_{k}\left(\phi_{0}(x)-f(x)\right)
$$

Note, entretanto, que para que a sequência $\left\{\phi_{k}(\cdot)\right\}$ satisfaça $(2.7) \operatorname{com} \lambda_{k}$ dado por (2.6), basta que as funções da sequência satisfaçam a desigualdade (2.4).

O lema que apresentamos a seguir nos mostra que se a função $f(\cdot)$ admite sequência de estimativas, é possível medir a taxa de convergência de $f\left(x^{k}\right) \rightarrow f\left(x^{*}\right)$ analisando apenas a velocidade com que $\lambda_{k} \rightarrow 0$.

Lema 2.3. Seja uma função $f(\cdot)$ que admite sequência de estimativa e $x^{*}$ uma solução do problema (2.1). Se uma sequência $\left\{x^{k}\right\}$ satisfaz

$$
f\left(x^{k}\right) \leq \phi_{k}^{*} \equiv \min \left\{\phi_{k}(x) \mid x \in \mathbb{R}^{n}\right\}
$$

então

$$
f\left(x^{k}\right)-f\left(x^{*}\right) \leq \lambda_{k}\left(\phi_{0}\left(x^{*}\right)-f\left(x^{*}\right)\right) \rightarrow 0 .
$$

Demonstração:

Como $f(\cdot)$ admite sequência de estimativa temos que para todo $x \in \mathbb{R}^{n}$

$$
\phi_{k}(x)-f(x) \leq \lambda_{k}\left(\phi_{0}(x)-f(x)\right) .
$$

Como $f\left(x^{k}\right) \leq \phi_{k}^{*} \leq \phi_{k}(x)$, temos que

$$
f\left(x^{k}\right)-f(x) \leq \lambda_{k}\left(\phi_{0}(x)-f(x)\right) .
$$

Em particular,

$$
f\left(x^{k}\right)-f\left(x^{*}\right) \leq \lambda_{k}\left(\phi_{0}\left(x^{*}\right)-f\left(x^{*}\right)\right)
$$

e da hipótese que $\lambda_{k} \rightarrow 0$, segue (2.9).

Na próxima seção apresentamos como Nesterov construiu a sequência $\left\{\phi_{k}(\cdot)\right\}$ de modo a satisfazer (2.4). 


\subsection{Construção da sequência $\left\{\phi_{k}(\cdot)\right\}$}

Na definição 2.4 abaixo, mostramos que Nesterov obtém cada função da sequência $\left\{\phi_{k}(\cdot)\right\}$ combinando a função anterior da sequência com uma aproximação da função $f(\cdot)$, como ilustrado na seguinte figura:

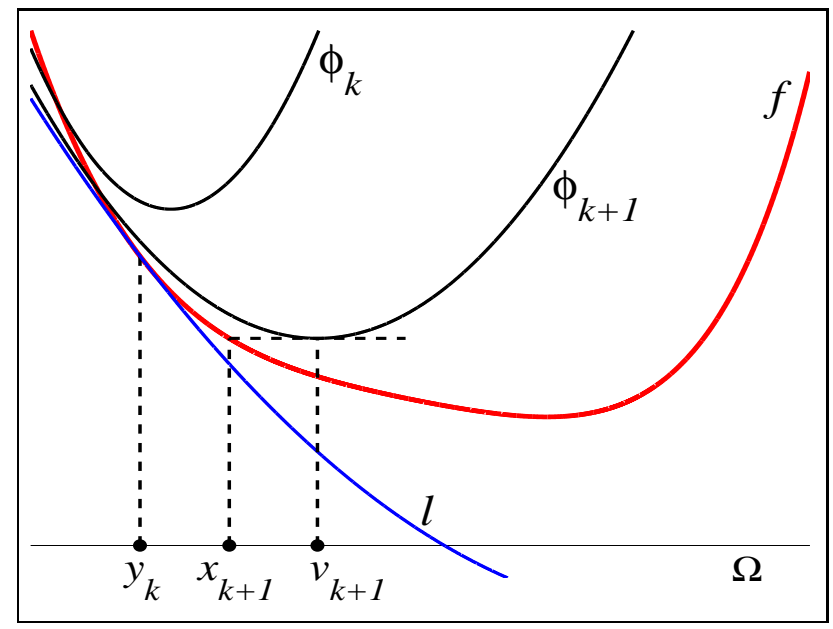

Figura 2.3: Construção de $\left\{\phi_{k}(\cdot)\right\}$

Definição 2.4. Sejam $y^{k} \in \mathbb{R}^{n}, \alpha_{k} \in(0,1), \phi_{0}(\cdot)$ uma função arbitrária em $\mathbb{R}^{n} e$ $f(\cdot)$ uma função convexa do $\mathbb{R}^{n}$. Para todo $x \in \mathbb{R}^{n}$ e todo $k \geq 0, \phi_{k}(\cdot)$ é definida por

$$
\begin{aligned}
\phi_{k+1}(x) & =\left(1-\alpha_{k}\right) \phi_{k}(x)+\alpha_{k} \ell_{k}\left(x, y^{k}\right) \\
\ell\left(x, y^{k}\right) & =f\left(y^{k}\right)+\left\langle\nabla f\left(y^{k}\right), x-y^{k}\right\rangle+\frac{\mu}{2}\left\|x-y^{k}\right\|^{2} .
\end{aligned}
$$

Se $f(\cdot)$ é fortemente convexa definimos $\mu$ como um limitante inferior do parâmetro de convexidade forte desta função, senão, definimos $\mu=0$.

No próximo lema, mostramos que a sequência de funções definidas acima satisfaz a desigualdade (2.4).

Lema 2.5. Considere a definição 2.4. Então,

$$
\phi_{k+1}(x)-f(x) \leq\left(1-\alpha_{k}\right)\left(\phi_{k}(x)-f(x)\right)
$$

Demonstração:

Por hipótese de $f(\cdot)$ ser convexa temos que $f(x) \geq \ell\left(x, y^{k}\right)$, para todo $x \in \mathbb{R}^{n}$. Da equação (2.10), temos que

$$
\phi_{k+1}(x) \leq\left(1-\alpha_{k}\right) \phi_{k}(x)+\alpha_{k} f(x) .
$$


Logo,

$$
\phi_{k+1}(x)-f(x) \leq\left(1-\alpha_{k}\right) \phi_{k}(x)+\alpha_{k} f(x)-f(x) .
$$

E portanto,

$$
\phi_{k+1}(x)-f(x) \leq\left(1-\alpha_{k}\right)\left(\phi_{k}(x)-f(x)\right) .
$$

No próximo lema veremos que se $\phi_{0}(\cdot)$ é uma função quadrática convexa simples e se a sequência $\left\{\phi_{k}(\cdot)\right\}$ é construída como definido em 2.4, então todas as funções da sequência $\left\{\phi_{k}(\cdot)\right\}$ são funções quadráticas convexas simples. Neste lema também são exibidos o minimizador e o valor ótimo de $\phi_{k+1}(\cdot)$.

Lema 2.6. Seja $\phi_{0}^{*} \in \mathbb{R}$ e $\gamma_{0}>0$, se

$$
\phi_{0}(x)=\phi_{0}^{*}+\frac{\gamma_{0}}{2}\left\|x-v^{0}\right\|^{2}
$$

então as funções da sequência $\left\{\phi_{k}(\cdot)\right\}$ definida por (2.10) e (2.11) admitem, para todo $k \geq 0$, a forma

$$
\phi_{k}(x)=\phi_{k}^{*}+\frac{\gamma_{k}}{2}\left\|x-v^{k}\right\|^{2}
$$

onde as sequências $\gamma_{k}$, v $v^{k} \phi_{k}^{*}$ são definidas por:

$$
\begin{aligned}
\gamma_{k+1}= & \left(1-\alpha_{k}\right) \gamma_{k}+\alpha_{k} \mu, \\
v^{k+1}= & \frac{1}{\gamma_{k+1}}\left(\left(1-\alpha_{k}\right) \gamma_{k} v^{k}+\alpha_{k} \mu y^{k}-\alpha_{k} \nabla f\left(y^{k}\right)\right), \\
\phi_{k+1}^{*}= & \left(1-\alpha_{k}\right) \phi_{k}^{*}+\alpha_{k} f\left(y^{k}\right)-\frac{\alpha_{k}^{2}}{2 \gamma_{k+1}}\left\|\nabla f\left(y^{k}\right)\right\|^{2} \\
& +\frac{\left(1-\alpha_{k}\right) \gamma_{k} \alpha_{k}}{\gamma_{k+1}}\left(\frac{\mu}{2}\left\|y^{k}-v^{k}\right\|^{2}+\left\langle\nabla f\left(y^{k}\right), v^{k}-y^{k}\right\rangle\right) .
\end{aligned}
$$

Demonstração:

Para provar que as funções $\phi_{k}(\cdot)$ obedecem (2.13), como elas são quadráticas, basta provar que a matriz hessiana é $\gamma_{k} I_{n}$. A demonstração é feita por indução. Note que $\nabla^{2} \phi_{0}(x)=\gamma_{0} I_{n}$. Assumimos, por hipótese de indução, que $\nabla^{2} \phi_{k}(x)=\gamma_{k} I_{n}$. Provaremos que $\nabla^{2} \phi_{k+1}(x)=\gamma_{k+1} I_{n}$ para todo $k \geq 0$. Das equações (2.10) e (2.11) temos que

$$
\nabla^{2} \phi_{k+1}(x)=\left(1-\alpha_{k}\right) \nabla^{2} \phi_{k}(x)+\alpha_{k} \mu I_{n}
$$


Usando a hipótese de indução temos

$$
\begin{aligned}
\nabla^{2} \phi_{k+1}(x) & =\left(1-\alpha_{k}\right) \gamma_{k} I_{n}+\alpha_{k} \mu I_{n} \\
& =\gamma_{k+1} I_{n} .
\end{aligned}
$$

Isto garante que $\phi_{k}(\cdot)$ tem a forma da equação (2.13) para todo $k \geq 0$.

Provaremos agora que o minimizador da função $\phi_{k+1}(\cdot)$ é $v^{k+1}$ dado por (2.15). Das equações (2.10) e (2.11)

$$
\phi_{k+1}(x)=\left(1-\alpha_{k}\right) \phi_{k}(x)+\alpha_{k}\left(f\left(y^{k}\right)+\left\langle\nabla f\left(y^{k}\right), x-y^{k}\right\rangle+\frac{\mu}{2}\left\|x-y^{k}\right\|^{2}\right) .
$$

E da primeira parte deste teorema sabemos que

$$
\begin{aligned}
\phi_{k+1}(x)= & \left(1-\alpha_{k}\right)\left(\phi_{k}^{*}+\frac{\gamma_{k}}{2}\left\|x-v^{k}\right\|^{2}\right) \\
& +\alpha_{k}\left(f\left(y^{k}\right)+\left\langle\nabla f\left(y^{k}\right), x-y^{k}\right\rangle+\frac{\mu}{2}\left\|x-y^{k}\right\|^{2}\right) .
\end{aligned}
$$

Da condição de otimalidade de $v^{k+1}$ para $\phi_{k+1}(\cdot)$ temos que:

$$
\left(1-\alpha_{k}\right) \gamma_{k}\left(v^{k+1}-v^{k}\right)+\alpha_{k} \nabla f\left(y^{k}\right)+\alpha_{k} \mu\left(v^{k+1}-y^{k}\right)=0 .
$$

Logo, isolando $v^{k+1}$, chegamos na seguinte expressão:

$$
v^{k+1}=\frac{1}{\left(1-\alpha_{k}\right) \gamma_{k}+\alpha_{k} \mu}\left(\left(1-\alpha_{k}\right) \gamma_{k} v^{k}+\alpha_{k}\left(\mu y^{k}-\nabla f\left(y^{k}\right)\right),\right.
$$

onde $\left(1-\alpha_{k}\right) \gamma_{k}+\alpha_{k} \mu=\gamma_{k+1}$. Agora, iremos explicitar o valor ótimo de $\phi_{k+1}(\cdot)$. Em particular, da primeira parte deste lema podemos escrever que para todo $k \geq 0$,

$$
\phi_{k+1}\left(y^{k}\right)=\phi_{k+1}^{*}+\frac{\gamma_{k+1}}{2}\left\|y^{k}-v^{k+1}\right\|^{2}
$$

Comparando esta expressão com a forma (2.10) avaliada em $y^{k} \in \mathbb{R}$, segue que

$$
\left(1-\alpha_{k}\right) \phi_{k}\left(y^{k}\right)+\alpha_{k} f\left(y^{k}\right)=\phi_{k+1}^{*}+\frac{\gamma_{k+1}}{2}\left\|y^{k}-v^{k+1}\right\|^{2}
$$

Assim, usando (2.13) chegamos na igualdade

$$
\left(1-\alpha_{k}\right)\left(\phi_{k}^{*}+\frac{\gamma_{k}}{2}\left\|y^{k}-v^{k}\right\|^{2}\right)+\alpha_{k} f\left(y^{k}\right)=\phi_{k+1}^{*}+\frac{\gamma_{k+1}}{2}\left\|y^{k}-v^{k+1}\right\|^{2} .
$$


Da expressão para $v^{k+1}$ temos que

$$
\begin{aligned}
v^{k+1}-y^{k} & =\frac{1}{\gamma_{k+1}}\left(\left(1-\alpha_{k}\right) \gamma_{k} v^{k}+\alpha_{k}\left(\mu y^{k}-\nabla f\left(y^{k}\right)\right)-\frac{\gamma_{k+1}}{\gamma_{k+1}} y^{k}\right. \\
& =\frac{1}{\gamma_{k+1}}\left(\left(1-\alpha_{k}\right) \gamma_{k} v^{k}+\alpha_{k} \mu y^{k}-\left(\left(1-\alpha_{k}\right) \gamma_{k}+\alpha_{k} \mu\right) y^{k}-\alpha_{k} \nabla f\left(y^{k}\right)\right) \\
& =\frac{1}{\gamma_{k+1}}\left(\left(1-\alpha_{k}\right) \gamma_{k} v^{k}-\left(1-\alpha_{k}\right) \gamma_{k} y^{k}-\alpha_{k} \nabla f\left(y^{k}\right)\right) \\
& =\frac{1}{\gamma_{k+1}}\left(\left(1-\alpha_{k}\right) \gamma_{k}\left(v^{k}-y^{k}\right)-\alpha_{k} \nabla f\left(y^{k}\right)\right) .
\end{aligned}
$$

Agora, note que

$$
\begin{aligned}
\left\|v^{k+1}-y^{k}\right\|^{2}= & \frac{1}{\gamma_{k+1}^{2}}\left(\left(1-\alpha_{k}\right)^{2} \gamma_{k}^{2}\left\|v^{k}-y^{k}\right\|^{2}\right. \\
& \left.-2\left(1-\alpha_{k}\right) \gamma_{k} \alpha_{k}\left\langle v^{k}-y^{k}, \nabla f\left(y^{k}\right)\right\rangle+\alpha_{k}^{2}\left\|\nabla f\left(y^{k}\right)\right\|^{2}\right) .
\end{aligned}
$$

Multiplicando por $\frac{\gamma_{k+1}}{2}$ obtemos que

$$
\begin{aligned}
\frac{\gamma_{k+1}}{2}\left\|v^{k+1}-y^{k}\right\|^{2}= & \frac{1}{2 \gamma_{k+1}}\left(\left(1-\alpha_{k}\right)^{2} \gamma_{k}^{2}\left\|v^{k}-y^{k}\right\|^{2}\right. \\
& \left.-2\left(1-\alpha_{k}\right) \gamma_{k} \alpha_{k}\left\langle v^{k}-y^{k}, \nabla f\left(y^{k}\right)\right\rangle+\alpha_{k}^{2}\left\|\nabla f\left(y^{k}\right)\right\|^{2}\right) .
\end{aligned}
$$

Substituindo em (2.18) podemos escrever a igualdade

$$
\begin{aligned}
\left(1-\alpha_{k}\right)\left(\phi_{k}^{*}+\frac{\gamma_{k}}{2}\left\|y^{k}-v^{k}\right\|^{2}\right)+\alpha_{k} f\left(y^{k}\right)= & \phi_{k+1}^{*}+\frac{1}{2 \gamma_{k+1}}\left(\left(1-\alpha_{k}\right)^{2} \gamma_{k}^{2}\left\|v^{k}-y^{k}\right\|^{2}\right. \\
& -2\left(1-\alpha_{k}\right) \gamma_{k} \alpha_{k}\left\langle v^{k}-y^{k}, \nabla f\left(y^{k}\right)\right\rangle \\
& \left.+\alpha_{k}^{2}\left\|\nabla f\left(y^{k}\right)\right\|^{2}\right) .
\end{aligned}
$$

Isolando $\phi_{k+1}^{*}$, é possível escrever a seguinte expressão

$$
\begin{aligned}
\phi_{k+1}^{*}= & \left(1-\alpha_{k}\right) \phi_{k}^{*}+\alpha_{k} f\left(y^{k}\right)-\frac{\alpha_{k}^{2}}{2 \gamma_{k+1}}\left\|\nabla f\left(y^{k}\right)\right\|^{2}+\left(1-\alpha_{k}\right) \frac{\gamma_{k}}{2}\left\|y^{k}-v^{k}\right\|^{2} \\
& -\frac{1}{2 \gamma_{k+1}}\left(\left(1-\alpha_{k}\right)^{2} \gamma_{k}^{2}\left\|v^{k}-y^{k}\right\|^{2}-2\left(1-\alpha_{k}\right) \gamma_{k} \alpha_{k}\left\langle v^{k}-y^{k}, \nabla f\left(y^{k}\right)\right\rangle\right) .
\end{aligned}
$$

Podemos reescrever os coeficientes do termo $\left\|v^{k}-y^{k}\right\|^{2}$ da seguinte forma:

$$
\begin{aligned}
\left(1-\alpha_{k}\right) \frac{\gamma_{k}}{2}-\frac{1}{2 \gamma_{k+1}}\left(1-\alpha_{k}\right)^{2} \gamma_{k}^{2} & =\left(1-\alpha_{k}\right) \frac{\gamma_{k}}{2}\left(1-\frac{\left(1-\alpha_{k}\right) \gamma_{k}}{\gamma_{k+1}}\right) \\
& =\left(1-\alpha_{k}\right) \frac{\gamma_{k}}{2}\left(\frac{\left(1-\alpha_{k}\right) \gamma_{k}+\alpha_{k} \mu-\left(1-\alpha_{k}\right) \gamma_{k}}{\gamma_{k+1}}\right) \\
& =\left(1-\alpha_{k}\right) \frac{\gamma_{k}}{2} \frac{\alpha_{k} \mu}{\gamma_{k+1}}
\end{aligned}
$$


E portanto,

$$
\begin{aligned}
\phi_{k+1}^{*}= & \left(1-\alpha_{k}\right) \phi_{k}^{*}+\alpha_{k} f\left(y^{k}\right)-\frac{\alpha_{k}^{2}}{2 \gamma_{k+1}}\left\|\nabla f\left(y^{k}\right)\right\|^{2} \\
& +\frac{\left(1-\alpha_{k}\right) \gamma_{k} \alpha_{k}}{\gamma_{k+1}}\left(\frac{\mu}{2}\left\|y^{k}-v^{k}\right\|^{2}+\left\langle\nabla f\left(y^{k}\right), v^{k}-y^{k}\right\rangle\right) .
\end{aligned}
$$

Na próxima seção mostramos como escolher $x^{k}, y^{k}$ e $\alpha_{k}$ de modo que em toda iteração $\phi_{k}^{*} \geq f\left(x^{k}\right)$.

\subsection{Escolhas das sequências que garantem otimali- dade}

Uma iteração qualquer do método sempre inicia com $x^{k}, v^{k} \in \mathbb{R}^{n}$ e $\gamma_{k} \in \mathbb{R}$. Então, escolhemos $\alpha_{k}$ sendo a maior raiz positiva da equação $L \alpha_{k}^{2}=\left(1-\alpha_{k}\right) \gamma_{k}+\alpha_{k} \mu$ e escolhemos um ponto intermediário $y^{k}$, como podemos ver na figura abaixo, a partir de $x^{k}$ na direção de $x^{k}$ para $v^{k}$. O tamanho do passo é dado por $\theta=\frac{\alpha_{k} \gamma_{k}}{\gamma_{k}+\alpha_{k} \mu}$.

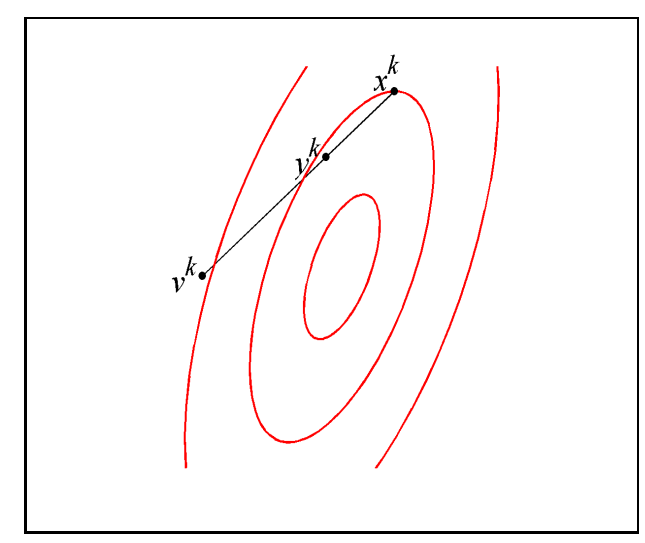

Figura 2.4: $y^{k}$ a partir de $x^{k}$ na direção $\left(v^{k}-x^{k}\right)$

Em um segundo momento, devemos determinar o próximo ponto $x^{k+1}$. Este ponto é obtido andando a partir de $y^{k}$, na direção oposta ao gradiente da função objetivo avaliado em $y^{k}$, e cujo tamanho do passo é dado por $\frac{1}{L}$. 


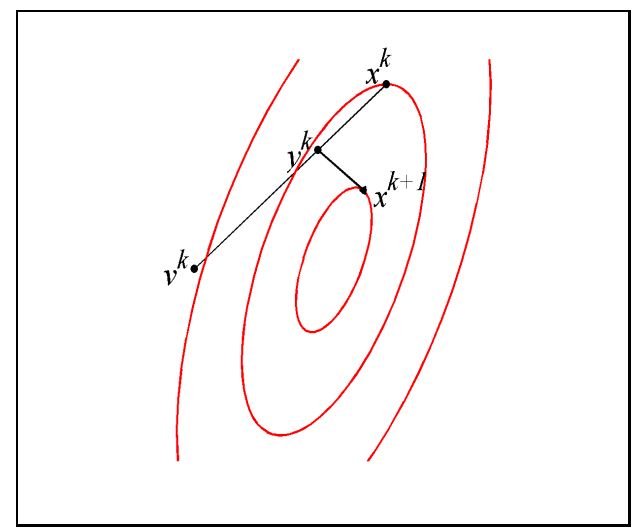

Figura 2.5: $x^{k+1}$ na direção $-\nabla f\left(y^{k}\right)$

Em seguida, devemos atualizar o valor de $\gamma_{k+1}$ e determinar o novo ponto $v^{k+1}$. Este ponto é encontrado, andando na direção oposta a $\nabla f\left(y^{k}\right)$ a partir do ponto $v^{k}$.

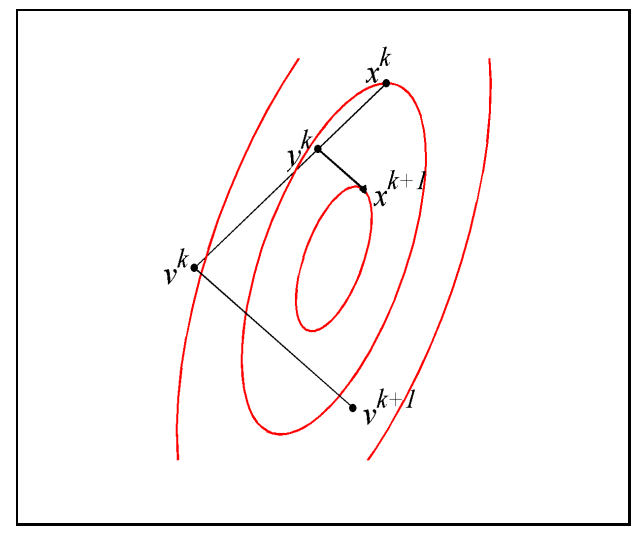

Figura 2.6: $v^{k+1}$ na direção $-\nabla f\left(y^{k}\right)$

O teorema 2.8, abaixo, nos mostra que as escolhas acima garantem $\phi_{k}^{*} \geq$ $f\left(x^{k}\right)$. Antes de provar este teorema, provamos a seguinte proposição:

Proposição 2.7. Se $f(\cdot)$ é uma função convexa com derivada Lipschitz contínua, com $L>0$ sendo o valor da constante de Lipschitz, e $x^{k+1}=y^{k}-\frac{1}{L} \nabla f\left(y^{k}\right)$, então

$$
f\left(y^{k}\right)-f\left(x^{k+1}\right) \geq \frac{1}{2 L}\left\|\nabla f\left(y^{k}\right)\right\|^{2}
$$

Demonstração:

Do teorema 1.15, a função

$$
h(x)=f\left(y^{k}\right)+\left\langle\nabla f\left(y^{k}\right), x-y^{k}\right\rangle+\frac{L}{2}\left\|x-y^{k}\right\|^{2},
$$


é tal que, para todo $x \in \mathbb{R}^{n}$,

$$
h(x)-f(x) \geq 0,
$$

Escrevendo a condição de um ponto ser o ponto ótimo para a função $h(\cdot)$, é fácil ver que ele é dado por $x^{k+1}=y^{k}-\frac{1}{L} \nabla f\left(y^{k}\right)$.

Observamos que,

$$
\begin{aligned}
h\left(x^{k+1}\right)-f\left(x^{k+1}\right) & =f\left(y^{k}\right)+\left\langle\nabla f\left(y^{k}\right), x^{k+1}-y^{k}\right\rangle+\frac{L}{2}\left\|x^{k+1}-y^{k}\right\|^{2}-f\left(x^{k+1}\right) \\
& =f\left(y^{k}\right)-f\left(x^{k+1}\right)+\left\langle\nabla f\left(y^{k}\right),-\frac{1}{L} \nabla f\left(y^{k}\right)\right\rangle+\frac{L}{2}\left\|-\frac{1}{L} \nabla f\left(y^{k}\right)\right\|^{2} \\
& =f\left(y^{k}\right)-f\left(x^{k+1}\right)-\frac{1}{L}\left\|\nabla f\left(y^{k}\right)\right\|^{2}+\frac{L}{2} \frac{1}{L^{2}}\left\|\nabla f\left(y^{k}\right)\right\|^{2} \\
& =f\left(y^{k}\right)-f\left(x^{k+1}\right)-\frac{1}{2 L}\left\|\nabla f\left(y^{k}\right)\right\|^{2} .
\end{aligned}
$$

De (2.20), podemos em particular dizer que

$$
h\left(x^{k+1}\right)-f\left(x^{k+1}\right) \geq 0 .
$$

De onde concluímos que

$$
f\left(y^{k}\right)-f\left(x^{k+1}\right) \geq \frac{1}{2 L}\left\|\nabla f\left(y^{k}\right)\right\|^{2} .
$$

Teorema 2.8. Seja $L>0$. Suponha que a iteração $k \geq 0$ começa com $\gamma_{k}>0$, $v^{k}, x^{k} \in \mathbb{R}^{n}$ tal que $\phi_{k}^{*} \geq f\left(x^{k}\right)$ e $\alpha_{k} \in(0,1)$ sendo a maior raíz da equação $L \alpha_{k}^{2}=$ $\left(1-\alpha_{k}\right) \gamma_{k}+\alpha_{k} \mu$. Se as seguintes escolhas são feitas

$$
y^{k}=x^{k}+\theta_{k}\left(v^{k}-x^{k}\right)
$$

$\operatorname{com} \theta_{k}=\frac{\alpha_{k} \gamma_{k}}{\gamma_{k}+\alpha_{k} \mu}$

$$
x^{k+1}=y^{k}-\frac{1}{L} \nabla f\left(y^{k}\right)
$$

$\gamma_{k+1}=\left(1-\alpha_{k}\right) \gamma_{k}+\alpha_{k} \mu$. Então a iteração termina com

$$
\phi_{k+1}^{*} \geq f\left(x^{k+1}\right)
$$


Demonstração:

Da igualdade (2.16) temos que

$$
\begin{aligned}
\phi_{k+1}^{*}= & \left(1-\alpha_{k}\right) \phi_{k}^{*}+\alpha_{k} f\left(y^{k}\right)-\frac{\alpha_{k}^{2}}{2 \gamma_{k+1}}\left\|\nabla f\left(y^{k}\right)\right\|^{2} \\
& +\frac{\alpha_{k}\left(1-\alpha_{k}\right) \gamma_{k}}{\gamma_{k+1}}\left(\frac{\mu}{2}\left\|y^{k}-v^{k}\right\|^{2}+\left\langle\nabla f\left(y^{k}\right), v^{k}-y^{k}\right\rangle\right) .
\end{aligned}
$$

Assim, pela hipótese que $\phi_{k}^{*} \geq f\left(x^{k}\right)$, segue que

$$
\begin{aligned}
\phi_{k+1}^{*} \geq & \left(1-\alpha_{k}\right) f\left(x^{k}\right)+\alpha_{k} f\left(y^{k}\right)-\frac{\alpha_{k}^{2}}{2 \gamma_{k+1}}\left\|\nabla f\left(y^{k}\right)\right\|^{2} \\
& +\frac{\alpha_{k}\left(1-\alpha_{k}\right) \gamma_{k}}{\gamma_{k+1}}\left(\frac{\mu}{2}\left\|y^{k}-v^{k}\right\|^{2}+\left\langle\nabla f\left(y^{k}\right), v^{k}-y^{k}\right\rangle\right)
\end{aligned}
$$

Note que $\frac{\alpha_{k}\left(1-\alpha_{k}\right) \gamma_{k}}{\gamma_{k+1}} \frac{\mu}{2}\left\|y^{k}-v^{k}\right\|^{2} \geq 0$, desta forma,

$$
\begin{aligned}
\phi_{k+1}^{*} \geq & \left(1-\alpha_{k}\right) f\left(x^{k}\right)+\alpha_{k} f\left(y^{k}\right)-\frac{\alpha_{k}^{2}}{2 \gamma_{k+1}}\left\|\nabla f\left(y^{k}\right)\right\|^{2} \\
& +\frac{\alpha_{k}\left(1-\alpha_{k}\right) \gamma_{k}}{\gamma_{k+1}}\left\langle\nabla f\left(y^{k}\right), v^{k}-y^{k}\right\rangle .
\end{aligned}
$$

Como toda função convexa diferenciável satisfaz

$$
f\left(x^{k}\right) \geq f\left(y^{k}\right)+\left\langle\nabla f\left(y^{k}\right), x^{k}-y^{k}\right\rangle
$$

temos que

$$
\left(1-\alpha_{k}\right) f\left(x^{k}\right) \geq\left(1-\alpha_{k}\right) f\left(y^{k}\right)+\left(1-\alpha_{k}\right)\left\langle\nabla f\left(y^{k}\right), x^{k}-y^{k}\right\rangle .
$$

Substituindo esta desigualdade em (2.23), chegamos que

$$
\begin{aligned}
\phi_{k+1}^{*} \geq & f\left(y^{k}\right)-\frac{\alpha_{k}^{2}}{2 \gamma_{k+1}}\left\|\nabla f\left(y^{k}\right)\right\|^{2} \\
& \left(1-\alpha_{k}\right)\left\langle\nabla f\left(y^{k}\right), \frac{\alpha_{k} \gamma_{k}}{\gamma_{k+1}}\left(v^{k}-y^{k}\right)+x^{k}-y^{k}\right\rangle .
\end{aligned}
$$

Provaremos agora, que $\frac{\alpha_{k} \gamma_{k}}{\gamma_{k+1}}\left(v^{k}-y^{k}\right)+x^{k}-y^{k}=0$. De $(2.21)$, segue que

$$
x^{k}-y^{k}=-\frac{\alpha_{k} \gamma_{k}}{\gamma_{k}+\alpha_{k} \mu}\left(v^{k}-x^{k}\right) .
$$


Assim, denotando por $\zeta=\frac{\alpha_{k} \gamma_{k}}{\gamma_{k+1}}\left(v^{k}-y^{k}\right)+x^{k}-y^{k}$, obtemos

$$
\zeta=\frac{\alpha_{k} \gamma_{k}}{\gamma_{k+1}}\left(v^{k}-y^{k}\right)-\frac{\alpha_{k} \gamma_{k}}{\gamma_{k}+\alpha_{k} \mu}\left(v^{k}-x^{k}\right)
$$

Da expressão para $\gamma_{k+1}$ podemos escrever $\gamma_{k+1}+\alpha_{k} \gamma_{k}=\gamma_{k}+\alpha_{k} \mu$. Logo,

$$
\begin{aligned}
\zeta & =\frac{\alpha_{k} \gamma_{k}}{\gamma_{k+1}}\left(v^{k}-y^{k}\right)-\frac{\alpha_{k} \gamma_{k}}{\gamma_{k+1}+\alpha_{k} \gamma_{k}}\left(v^{k}-x^{k}\right) \\
& =\frac{\alpha_{k} \gamma_{k}}{\gamma_{k+1}}\left(\left(v^{k}-y^{k}\right)-\frac{\gamma_{k+1}}{\gamma_{k+1}+\alpha_{k} \gamma_{k}}\left(v^{k}-x^{k}\right)\right) .
\end{aligned}
$$

Subtituindo (2.21), temos que

$$
\begin{aligned}
\zeta & =\frac{\alpha_{k} \gamma_{k}}{\gamma_{k+1}}\left(\left(v^{k}-x^{k}\right)-\frac{\alpha_{k} \gamma_{k}}{\gamma_{k}+\alpha_{k} \mu}\left(v^{k}-x^{k}\right)-\frac{\gamma_{k+1}}{\gamma_{k+1}+\alpha_{k} \gamma_{k}}\left(v^{k}-x^{k}\right)\right) . \\
& =\frac{\alpha_{k} \gamma_{k}}{\gamma_{k+1}}\left(\left(v^{k}-x^{k}\right)-\left(\frac{\alpha_{k} \gamma_{k}}{\gamma_{k+1}+\alpha_{k} \gamma_{k}}\left(v^{k}-x^{k}\right)+\frac{\gamma_{k+1}}{\gamma_{k+1}+\alpha_{k} \gamma_{k}}\left(v^{k}-x^{k}\right)\right)\right) . \\
& =\frac{\alpha_{k} \gamma_{k}}{\gamma_{k+1}}\left(\left(v^{k}-x^{k}\right)-\left(\frac{\gamma_{k+1}+\alpha_{k} \gamma_{k}}{\gamma_{k+1}+\alpha_{k} \gamma_{k}}\left(v^{k}-x^{k}\right)\right)\right) . \\
& =0
\end{aligned}
$$

Então, (2.24) implica que

$$
\phi_{k+1}^{*} \geq f\left(y^{k}\right)-\frac{\alpha_{k}^{2}}{2 \gamma_{k+1}}\left\|\nabla f\left(y^{k}\right)\right\|^{2}
$$

Mas, pela proposição 2.7, temos que se $x^{k+1}$ é determinado a partir de $y^{k}$ satisfazendo (2.22) podemos garantir o seguinte decréscimo

$$
f\left(y^{k}\right)-f\left(x^{k+1}\right) \geq \frac{1}{2 L}\left\|\nabla f\left(y^{k}\right)\right\|^{2} .
$$

Substituindo este fato em (2.25), obtemos que

$$
\phi_{k+1}^{*} \geq f\left(x^{k+1}\right)+\frac{1}{2 L}\left\|\nabla f\left(y^{k}\right)\right\|^{2}-\frac{\alpha_{k}^{2}}{2 \gamma_{k+1}}\left\|\nabla f\left(y^{k}\right)\right\|^{2}
$$

Como $\alpha_{k}$ satisfaz $\alpha_{k}^{2}=\frac{\gamma_{k+1}}{L}$, os coefientes dos termos $\left\|\nabla f\left(y^{k}\right)\right\|^{2}$ se anulam e portanto

$$
\phi_{k+1}^{*} \geq f\left(x^{k+1}\right)
$$

Provaremos agora que a maior raíz da equação $\alpha_{k}^{2}=\frac{\left(1-\alpha_{k}\right) \gamma_{k}+\alpha_{k} \mu}{L}$ pertence a $(0,1)$. 
Como $\mu<L$ e $L>0$, temos que

$$
\frac{4 \mu}{L}<4
$$

Somando $\frac{4 \gamma_{k}}{L}$ nos dois lados desta desigualdade, chegamos que

$$
\frac{4 \gamma_{k}}{L}<4-\frac{4 \mu}{L}+\frac{4 \gamma_{k}}{L}
$$

Somando agora $\left(\frac{\gamma_{k}-\mu}{L}\right)^{2}$, obtemos

$$
\left(\frac{\gamma_{k}-\mu}{L}\right)^{2}+\frac{4 \gamma_{k}}{L}<4-\frac{4 \mu}{L}+\frac{4 \gamma_{k}}{L}+\left(\frac{\gamma_{k}-\mu}{L}\right)^{2} .
$$

De onde podemos escrever que

$$
\begin{aligned}
\left(\frac{\gamma_{k}-\mu}{L}\right)^{2}+\frac{4 \gamma_{k}}{L} & <4+\frac{4\left(\gamma_{k}-\mu\right)}{L}+\left(\frac{\gamma_{k}-\mu}{L}\right)^{2} \\
& <\left(2+\left(\frac{\gamma_{k}-\mu}{L}\right)\right)^{2}
\end{aligned}
$$

Como $\left(\frac{\gamma_{k}-\mu}{L}\right)^{2}+\frac{4 \gamma_{k}}{L}>0$, segue que

$$
\sqrt{\left(\frac{\gamma_{k}-\mu}{L}\right)^{2}+\frac{4 \gamma_{k}}{L}}<\left(2+\left(\frac{\gamma_{k}-\mu}{L}\right)\right) .
$$

Logo,

$$
-\left(\frac{\gamma_{k}-\mu}{L}\right)+\sqrt{\left(\frac{\gamma_{k}-\mu}{L}\right)^{2}+\frac{4 \gamma_{k}}{L}}<2
$$

Ou seja,

$$
\frac{-\left(\frac{\gamma_{k}-\mu}{L}\right)+\sqrt{\left(\frac{\gamma_{k}-\mu}{L}\right)^{2}+\frac{4 \gamma_{k}}{L}}}{2}<1 .
$$

Mas observe que o lado esquerdo dessa desigualdade é exatamente a maior raíz da equação $\alpha_{k}^{2}=\frac{\left(1-\alpha_{k}\right) \gamma_{k}+\alpha_{k} \mu}{L}$. Como é perceptível ver também que esta raíz é positiva, $\alpha_{k} \in(0,1)$.

Podemos então sintetizar o método de Nesterov da seguinte maneira: 


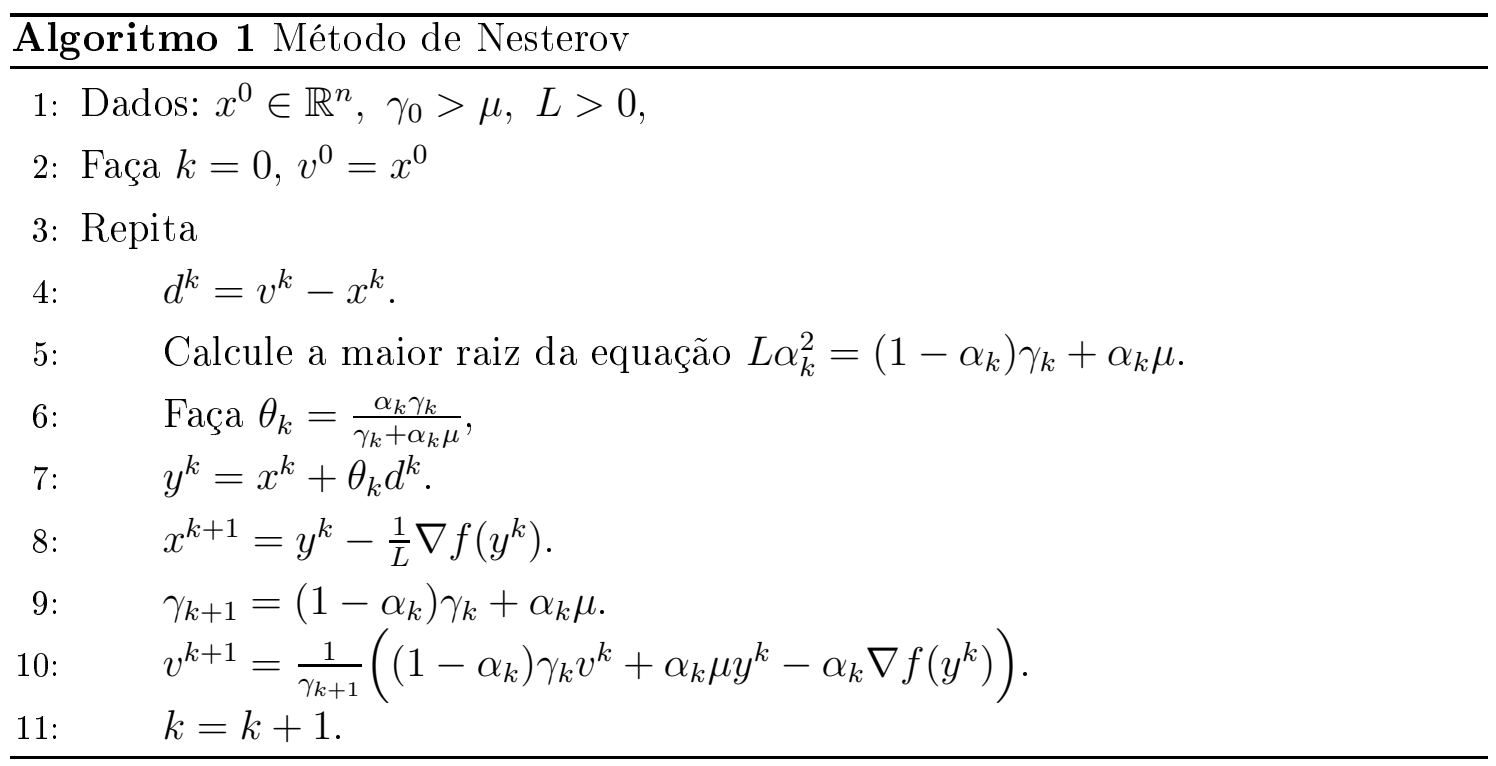

\subsection{Otimalidade do método}

Nas sessões 2.3 e 2.2 mostramos como construir a sequência aproximadora $\left\{\phi_{k}(\cdot)\right\}$ e como escolher os termos das sequências $x^{k}, y^{k}, v^{k}$ e $\alpha_{k}$ para garantir as desigualdades (2.4) e $\phi_{k}^{*} \geq f\left(x^{k}\right)$. Desta forma é possível escrever

$$
f\left(x^{k}\right)-f\left(x^{*}\right) \leq \lambda_{k}\left(\phi_{0}\left(x^{*}\right)-f\left(x^{*}\right)\right) .
$$

Nessa seção, mostramos os resultados apresentados por Nesterov que garantem a otimalidade do método.

Lema 2.9. Se $\lambda_{0}=1$ e no algoritmo $1 \gamma_{0} \geq \mu$, então

$$
\lambda_{k} \leq \min \left\{\left(1-\sqrt{\frac{\mu}{L}}\right)^{k}, \frac{4 L}{\left(2 \sqrt{L}+k \sqrt{\gamma_{0}}\right)^{2}}\right\} .
$$

Demonstração:

Por indução, provaremos que $\gamma_{k} \geq \mu$, para todo $k \geq 0$. Por hipótese, $\gamma_{0} \geq \mu$. Assumimos por hipótese de indução que $\gamma_{k} \geq \mu$. Logo

$$
\gamma_{k+1}=\left(1-\alpha_{k}\right) \gamma_{k}+\alpha_{k} \mu \geq \mu
$$


Como $\alpha_{k}^{2}=\frac{\gamma_{k+1}}{L}$, usando o fato anterior $\alpha_{k}^{2} \geq \frac{\mu}{L}$. Logo

$$
\begin{aligned}
\alpha_{k} & \geq \sqrt{\frac{\mu}{L}} \\
\left(1-\alpha_{k}\right) & \leq\left(1-\sqrt{\frac{\mu}{L}}\right) .
\end{aligned}
$$

Como $\lambda_{k}=\prod_{i=0}^{k-1}\left(1-\alpha_{i}\right)$, concluímos que $\lambda_{k} \leq\left(1-\sqrt{\frac{\mu}{L}}\right)^{k}$. Isto prova a primeira desigualdade. Provaremos agora a segunda desigualdade.

Afirmamos que $\gamma_{k} \geq \gamma_{0} \lambda_{k}$. Como $\lambda_{0}=1, \gamma_{0}=\gamma_{0} \lambda_{0}$. Assumiremos como hipótese de indução que $\gamma_{k} \geq \gamma_{0} \lambda_{k}$.

$$
\begin{aligned}
\gamma_{k+1} & \geq\left(1-\alpha_{k}\right) \gamma_{k} \\
& \geq\left(1-\alpha_{k}\right) \gamma_{0} \lambda_{k}=\gamma_{0} \lambda_{k+1}
\end{aligned}
$$

Desta forma $L \alpha_{k}^{2}=\gamma_{k+1} \geq \gamma_{0} \lambda_{k+1}$.

Agora, denote $a_{k}=\frac{1}{\sqrt{\lambda_{k}}}$. Desde que $\left\{\lambda_{k}\right\}$ é uma sequência decrescente, temos:

$$
\begin{aligned}
a_{k+1}-a_{k} & =\frac{1}{\sqrt{\lambda_{k+1}}}-\frac{1}{\sqrt{\lambda_{k}}} \\
& =\frac{\sqrt{\lambda_{k}}-\sqrt{\lambda_{k+1}}}{\sqrt{\lambda_{k+1}} \sqrt{\lambda_{k}}}
\end{aligned}
$$

multiplicando e dividindo a direita por $\left(\sqrt{\lambda_{k}}+\sqrt{\lambda_{k+1}}\right)$ temos que:

$$
a_{k+1}-a_{k}=\frac{\lambda_{k}-\lambda_{k+1}}{\sqrt{\lambda_{k+1} \lambda_{k}}\left(\sqrt{\lambda_{k}}+\sqrt{\lambda_{k+1}}\right)} .
$$

Agora, note que

$$
\sqrt{\lambda_{k} \lambda_{k+1}}\left(\sqrt{\lambda_{k}}+\sqrt{\lambda_{k+1}}\right)=\sqrt{\lambda_{k}^{2} \lambda_{k+1}}+\sqrt{\lambda_{k} \lambda_{k+1}^{2}},
$$

mas, do fato que $\lambda_{k} \geq \lambda_{k+1}$, segue que

$$
\begin{aligned}
\sqrt{\lambda_{k} \lambda_{k+1}}\left(\sqrt{\lambda_{k}}+\sqrt{\lambda_{k+1}}\right) & \leq \lambda_{k} \sqrt{\lambda_{k+1}}+\sqrt{\lambda_{k}^{2} \lambda_{k+1}} \\
& =2 \lambda_{k} \sqrt{\lambda_{k+1}} .
\end{aligned}
$$

Então, podemos afirmar que

$$
\frac{1}{\sqrt{\lambda_{k+1} \lambda_{k}}\left(\sqrt{\lambda_{k}}+\sqrt{\lambda_{k+1}}\right)} \geq \frac{1}{2 \lambda_{k} \sqrt{\lambda_{k+1}}}
$$


Logo,

$$
\begin{aligned}
a_{k+1}-a_{k} \geq \frac{\lambda_{k}-\lambda_{k+1}}{2 \lambda_{k} \sqrt{\lambda_{k+1}}} & =\frac{\lambda_{k}-\left(1-\alpha_{k}\right) \lambda_{k}}{2 \lambda_{k} \sqrt{\lambda_{k+1}}} \\
& =\frac{\alpha_{k}}{2 \sqrt{\lambda_{k+1}}}
\end{aligned}
$$

Como $L \alpha_{k}^{2} \geq \gamma_{0} \lambda_{k+1}$, temos que

$$
a_{k+1}-a_{k} \geq \frac{1}{2} \sqrt{\frac{\gamma_{0}}{L}}
$$

Temos então que $a_{k+1}-a_{k} \geq \frac{1}{2} \sqrt{\frac{\gamma_{0}}{L}}$. Então $a_{k}-a_{0} \geq \frac{k}{2} \sqrt{\frac{\gamma_{0}}{L}}$. Ou seja

$$
\begin{aligned}
a_{k} & \geq 1+\frac{k}{2} \sqrt{\frac{\gamma_{0}}{L}} \\
\frac{1}{\sqrt{\lambda_{k}}} & \geq \frac{2 \sqrt{L}+k \sqrt{\gamma_{0}}}{2 \sqrt{L}} \\
\lambda_{k} & \leq \frac{4 L}{\left(2 \sqrt{L}+k \sqrt{\gamma_{0}}\right)^{2}} .
\end{aligned}
$$

Isto prova a segunda desigualdade.

O lema que provamos abaixo fornece, juntamente com o lema anterior, ferramentas para provar a otimalidade do método.

Lema 2.10. Sejam $\phi_{0}^{*}=f\left(x^{0}\right), \lambda_{0}=1$ e $\lambda_{k}=\prod_{i=0}^{k-1}\left(1-\alpha_{i}\right)$. O algoritmo 1 gera uma sequência $\left\{x^{k}\right\}$ tal que

$$
f\left(x^{k}\right)-f\left(x^{*}\right) \leq \lambda_{k}\left[f\left(x^{0}\right)-f\left(x^{*}\right)+\frac{\gamma_{0}}{2}\left\|x^{0}-x^{*}\right\|^{2}\right] .
$$

Demonstração:

Como valem (2.12) e (2.8), podemos garantir que

$$
f\left(x^{k}\right)-f\left(x^{*}\right) \leq \lambda_{k}\left(\phi_{0}\left(x^{*}\right)-f\left(x^{*}\right)\right) .
$$

Porém, como $\phi_{0}^{*}=f\left(x^{0}\right)$, podemos escrever $\phi_{0}\left(x^{*}\right)=f\left(x^{0}\right)+\frac{\gamma_{0}}{2}\left\|x^{*}-x^{0}\right\|^{2}$. Portanto

$$
f\left(x^{k}\right)-f\left(x^{*}\right) \leq \lambda_{k}\left(f\left(x^{0}\right)+\frac{\gamma_{0}}{2}\left\|x-x^{0}\right\|^{2}-f\left(x^{*}\right)\right) .
$$


No próximo teorema provamos que o método de Nesterov é ótimo.

Teorema 2.11. O algoritmo 1 gera uma sequência $\left\{x^{k}\right\}$ tal que

$$
f\left(x^{k}\right)-f\left(x^{*}\right) \leq \min \left\{\left(1-\sqrt{\frac{\mu}{L}}\right)^{k}, \frac{4 L}{\left(2 \sqrt{L}+k \sqrt{\gamma_{0}}\right)^{2}}\right\}\left(\frac{\left(L+\gamma_{0}\right)}{2}\left\|x^{0}-x^{*}\right\|^{2}\right) .
$$

Demonstração:

Como $f(\cdot)$ tem gradiente Lipchitz contínuo vale que:

$$
f\left(x^{0}\right)-f\left(x^{*}\right)-\left\langle\nabla f\left(x^{*}\right), x^{0}-x^{*}\right\rangle \leq \frac{L}{2}\left\|x^{0}-x^{*}\right\|^{2} .
$$

Como $x^{*}$ é o minimizador irrestrito de $f(\cdot), \nabla f\left(x^{*}\right)=0$, logo

$$
f\left(x^{0}\right)-f\left(x^{*}\right) \leq \frac{L}{2}\left\|x^{0}-x^{*}\right\|^{2} .
$$

Pelo lema 2.10, podemos escrever

$$
f\left(x^{k}\right)-f\left(x^{*}\right) \leq \lambda_{k}\left(\frac{\left(L+\gamma_{0}\right)}{2}\left\|x^{0}-x^{*}\right\|^{2}\right) .
$$

Portanto, pelo lema 2.9 segue que

$$
f\left(x^{k}\right)-f\left(x^{*}\right) \leq \min \left\{\left(1-\sqrt{\frac{\mu}{L}}\right)^{k}, \frac{4 L}{\left(2 \sqrt{L}+k \sqrt{\gamma_{0}}\right)^{2}}\right\}\left(\frac{\left(L+\gamma_{0}\right)}{2}\left\|x^{0}-x^{*}\right\|^{2}\right) .
$$

Deste teorema, obtemos que

$$
f\left(x^{k}\right)-f\left(x^{*}\right)=O\left(\frac{1}{k^{2}}\right)
$$

Como vimos no primeiro capítulo, isto quer dizer que uma solução ótima $x^{*}$ pode ser estimada, com precisão $\epsilon$ em um número proporcional a $\frac{1}{\sqrt{\epsilon}}$ iterações, garantindo assim que o método é ótimo.

No próximo capítulo apresentaremos dois métodos ótimos para problemas de otimização de uma função convexa restrita a um conjunto convexo. Em seguida, apresentamos um novo método ótimo desenvolvido durante este trabalho. 


\section{Capítulo 3}

\section{Métodos para problemas com restrições}

Considere o problema de otimização convexa restrita

$$
\begin{aligned}
& \underset{x \in C}{\operatorname{minimizar}} \\
& \mathrm{x}
\end{aligned}
$$

em que $C$ é um conjunto não vazio, convexo e fechado em $\mathbb{R}^{n}, f: \mathbb{R}^{n} \rightarrow \mathbb{R}$ é convexa e diferenciável em $C$ e o gradiente da função objetivo é Lipschitz contínuo em $C$ com constante de Lipschitz $L>0$.

Neste capítulo apresentamos inicialmente o método ótimo desenvolvido por Nesterov [15] para resolver o problema acima. Em seguida, discutimos o método ótimo construído por Auslender e Teboulle [1] que será usado como base para um novo método ótimo que desenvolvemos no final do capítulo.

\subsection{O método de Nesterov}

Para lidar com restrições, Nesterov estendeu as ideias do capítulo anterior para o seguinte problema,

$$
\underset{x \in \mathbb{R}^{n}}{\operatorname{minimizar}} F(x)
$$

em que $F(x)=f(x)+G(x)$ e $G(\cdot)$ é a função indicadora do conjunto $C$.

As diferenças entre o método irrestrito com o método restrito são:

- os pontos $v^{k}$ e $x^{k}$ são obtidos através de projeções no conjunto viável; 
- a aproximação $l(\cdot, \cdot)$ é feita no ponto $x^{k+1}$ e aproxima apenas a função $f(\cdot)$;

- é possível calcular uma estimativa para o valor da constante de Lipschitz que pode ser usada pelo método sem perder a otimalidade. Para isto Nesterov introduziu o conceito de mapeamento gradiente que apresentamos abaixo.

\subsubsection{Mapeamento Gradiente}

O mapeamento gradiente é o argumento que minimiza uma combinação entre uma aproximação convexa de $f(\cdot)$ em um ponto $y \in \mathbb{R}^{n}$ e a função indicadora do conjunto $C$.

Definição 3.1. Sejam $x, y \in \mathbb{R}^{n}$ e $L^{a}>0$. Define-se o mapeamento gradiente por

$$
T_{L^{a}}(y)=\operatorname{argmin}_{x \in \mathbb{R}^{n}} m_{L^{a}}(y ; x),
$$

onde $m_{L^{a}}(y ; x)=f(y)+\langle\nabla f(y), x-y\rangle+\frac{L^{a}}{2}\|x-y\|^{2}+G(x)$.

É fácil observar que, se o conjunto viável é todo espaço $\mathbb{R}^{n}, T_{L^{a}}(y)$ é um ponto a partir de $y$ na direção oposta ao gradiente de $f(\cdot)$, com o passo $\frac{1}{L^{a}}$, como pode ser visto na demonstração da proposição 2.7 . No caso de $G(\cdot)$ ser a função indicadora de um conjunto convexo e fechado de $\mathbb{R}^{n}, T_{L^{a}}(y)$ é a projeção ortogonal do ponto descrito acima no conjunto viável.

Com estas definições e o seguinte subgradiente de $F(\cdot)$

$$
F^{\prime}(z)=\nabla f(z)+\zeta_{G}(z)
$$

em que $\zeta_{G}(z)=L^{a}\left(z-T_{L}(z)\right)-\nabla f(z) \in \partial G(z)$, Nesterov conseguiu provar o lema abaixo que fornece um critério para estimar o valor de $L$.

Lema 3.2. Se $L^{a} \geq L$ então

$$
\left\langle F^{\prime}\left(T_{L^{a}}(y)\right), y-T_{L^{a}}(y)\right\rangle \geq \frac{1}{L^{a}}\left\|F^{\prime}\left(T_{L^{a}}(y)\right)\right\|^{2} .
$$

A demonstração desse lema e uma descrição mais detalhada deste método podem ser encontradas em [15]. Abaixo, apresentamos um esquema do método de Nesterov. 


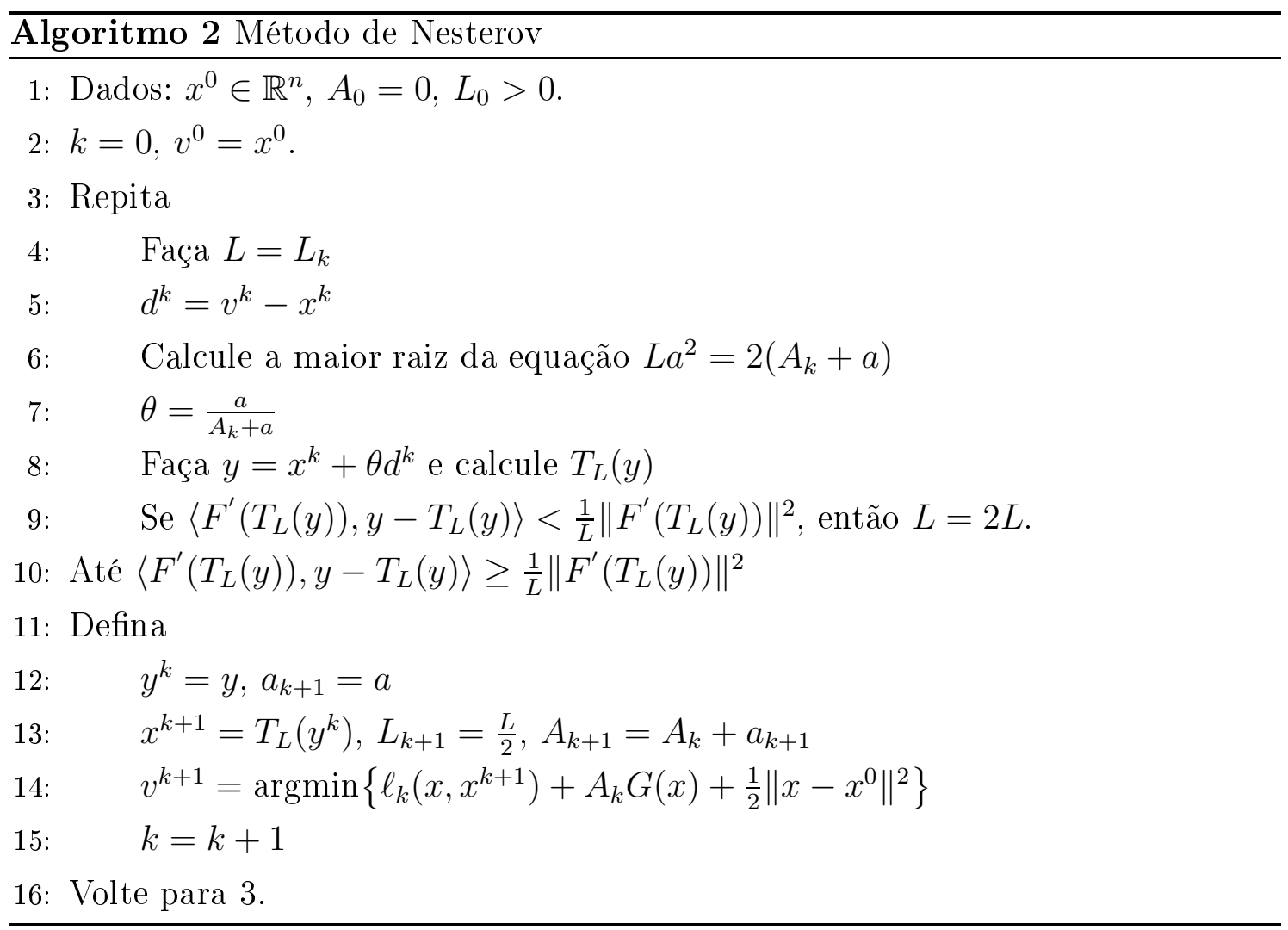

Também em [15], Nesterov demonstra que este método é ótimo para a classe de funções convexas. Além disso, como em cada iteração do loop interno é preciso avaliar duas vezes o gradiente, Nesterov estimou que o número de avaliações depois de $k$ iterações é:

$$
N_{k} \leq 4(k+1)+\log _{2} \frac{L}{L_{0}}
$$

Isso garante que o laço interno será feito em um número finito e constante de vezes.

\subsection{O método de Auslender e Teboulle}

Inspirados no método de Nesterov, Auslender e Teboulle desenvolveram um algoritmo para resolver o problema (3.1). Para isto, usaram uma sequência de funções $\left\{\phi_{k}(\cdot)\right\}$ que guardam informações sobre o conjunto viável através de funções distâncias não convencionais conhecidas como distâncias de Bregman. Essas distâncias de Bregman estão definidas no interior do conjunto viável e funcionam como barreiras, impedindo que a sequência de iterados geradas pelo algoritmo saiam desse conjunto. Dessa forma, os iterados estão sempre no interior do conjunto viável. 
Nessa seção mostramos como estender esse método usando distâncias de Bregman definidas em um conjunto maior que o conjunto viável, contemplando, assim, o caso da distância euclidiana ao quadrado e permitindo que um iterado atinja a borda do conjunto viável. Análogo a Nesterov, mostramos que é possível estabelecer um critério para determinar aproximadamente o valor da constante de Lipschitz de forma adaptativa.

Como vimos no capítulo anterior, a ideia de Nesterov é construir uma sequência de funções $\left\{\phi_{k}(\cdot)\right\}$ e uma sequência $\left\{x^{k}\right\} \subset C$ minimizante para $f(\cdot)$ de modo que seja possível escrever a seguinte desigualdade,

$$
f\left(x^{k}\right)-f\left(x^{*}\right) \leq \lambda_{k}\left(\phi_{0}\left(x^{*}\right)-f\left(x^{*}\right)\right)
$$

$\operatorname{com} \lambda_{k}:=\prod_{i=0}^{k-1}\left(1-\alpha_{i}\right)$

Como estamos tratando de otimização em um conjunto convexo $C$, para garantir a desigualdade (3.3), basta que as sequências $\left\{\phi_{k}(\cdot)\right\}$ e $\left\{x^{k}\right\}$ satisfaçam as seguintes condições:

- Para todo $x \in C$

$$
\phi_{k+1}(x)-f(x) \leq\left(1-\alpha_{k}\right)\left(\phi_{k}(x)-f(x)\right)
$$

- Para todo $k \geq 0$

$$
\phi_{k}^{*} \geq \min \left\{\phi_{k}(x) \mid x \in C\right\} \geq f\left(x^{k}\right) .
$$

Note que, no capítulo que estavámos tratando do caso irrestrito, tínhamos condições equivalentes a estas mas que deveriam ser válidas em todo espaço $\mathbb{R}^{n}$. Além disso, vimos que as funções da sequência $\left\{\phi_{k}(\cdot)\right\}$ são funções quadráticas convexas simples com $D\left(x, v^{k}\right)=\left\|x-v^{k}\right\|^{2}$. Neste capítulo mostramos como Auslender e Teboulle construíram as sequências $\left\{\phi_{k}(\cdot)\right\}$ de forma equivalente a Nesterov porém trocando a função distância euclidiana ao quadrado por um distância não convencional. Em seguida determinamos $\left\{x^{k}\right\}$ e mostramos que a construção dessas duas sequências satisfaz as duas desigualdes acima. Para isso precisamos introduzir o conceito de distâncias de Bregman. 


\subsubsection{Distâncias de Bregman Coercivas}

As definições e resultados desta seção seguiram as referências [1, 16].

Definição 3.3. Seja $\Omega$ um conjunto convexo, aberto e não vazio do $\mathbb{R}^{n}$. Seja $h$ : $\bar{\Omega} \rightarrow \mathbb{R}$ uma função própria, semicontinua inferior, estritamente convexa, contínua em $\bar{\Omega}$ e diferenciável em $\Omega$. Defina

$$
D_{h}(x, y)=h(x)-h(y)-\langle\nabla h(y), x-y\rangle(>0 \text { para } x \neq y) .
$$

A função $h(\cdot)$ é dita uma função de Bregman e $D_{h}(\cdot, \cdot)$ a distância de Bregman induzida por $h(\cdot)$ se valem

B1. (i) Para todo $x \in \bar{\Omega}, D_{h}(x, \cdot)$ tem conjuntos de nivel limitados em $\Omega$;

(ii) Para todo $y \in \Omega, D_{h}(\cdot, y)$ tem conjuntos de nível limitados em $\bar{\Omega}$;

B2. Para todo $y \in \bar{\Omega}$, para todo $\left\{y^{k}\right\} \subset \Omega$ com $\lim _{k \rightarrow \infty} y^{k}=y$, tem-se $\lim _{k \rightarrow \infty} D_{h}\left(y, y^{k}\right)=0 ;$

B3. Se $\left\{y^{k}\right\}$ é uma sequência limitada em $\Omega$ e $y \in \bar{\Omega}$ tal que $\lim _{k \rightarrow \infty} D_{h}\left(y, y^{k}\right)=0$, então $y=\lim _{k \rightarrow \infty} y^{k}$.

O conjunto $\Omega$ é chamado zona de $h(\cdot)$.

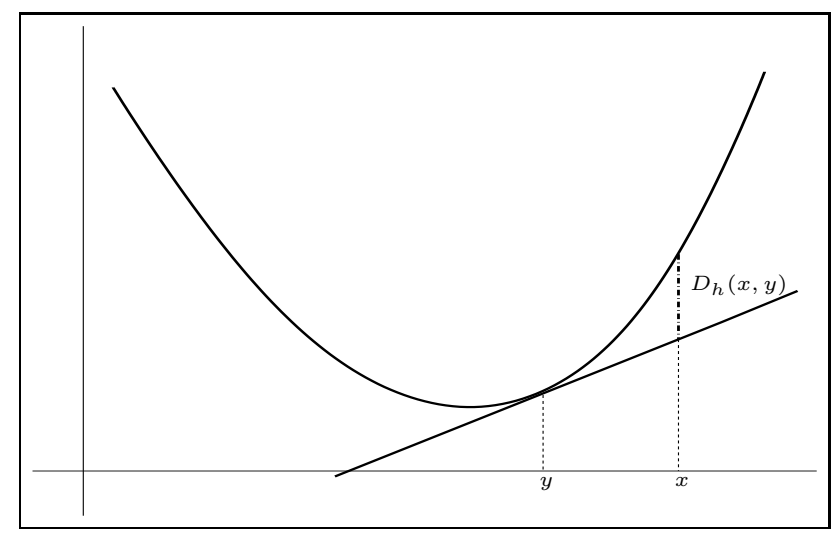

Figura 3.1: Distância de Bregman de $x$ em relação a $y$

Para todo $x, y \in \Omega$, o gradiente de $D_{h}(x, y)$ com relação a primeira variável é dado por $\nabla_{1} D_{h}(x, y)=\nabla h(x)-\nabla h(y)$.

Uma propriedade das distâncias de Bregman que será útil na construção da sequência $\left\{\phi_{k}(\cdot)\right\}$ é o fato de satisfazerem a Identidade dos três pontos. 
Proposição 3.4. (Identidade dos três pontos) Se h(·) é uma função de Bregman com zona $\Omega$, então, para todo $x \in \bar{\Omega}$ e $y, z \in \Omega$, satisfaz a identidade dos três pontos:

$$
D_{h}(x, y)=D_{h}(x, z)+D_{h}(z, y)+\left\langle\nabla_{1} D_{h}(z, y), x-z\right\rangle
$$

Demonstração:

Por definição,

$$
D_{h}(x, y)=h(x)-h(y)-\langle\nabla h(y), x-y\rangle
$$

Que pode ser reescrito como,

$$
\begin{aligned}
D_{h}(x, y)= & h(x)-h(y)-\langle\nabla h(y), x-y\rangle+(-h(z)+h(z)) \\
& -\langle\nabla h(z), x-z\rangle+\langle\nabla h(z), x-z\rangle \\
& -\langle\nabla h(y), z-y\rangle+\langle\nabla h(y), z-y\rangle .
\end{aligned}
$$

Rearranjando os termos temos que,

$$
\begin{aligned}
D_{h}(x, y)= & h(x)-h(z)-\langle\nabla h(z), x-z\rangle \\
& +h(z)-h(y)-\langle\nabla h(y), z-y\rangle \\
& -\langle\nabla h(y), x-y\rangle+\langle\nabla h(z), x-z\rangle+\langle\nabla h(y), z-y\rangle .
\end{aligned}
$$

E assim,

$$
\begin{aligned}
D_{h}(x, y)= & D_{h}(x, z)+D_{h}(z, y) \\
& +\langle\nabla h(z), x-z\rangle+\langle\nabla h(y),-(x-y)+z-y\rangle .
\end{aligned}
$$

E portanto,

$$
\begin{aligned}
D_{h}(x, y)= & D_{h}(x, z)+D_{h}(z, y) \\
& +\langle\nabla h(z), x-z\rangle+\langle\nabla h(y),-x+z\rangle .
\end{aligned}
$$

De onde segue a equação (3.6). 
No método de Auslender e Teboulle o conjunto viável está implícito na distância de Bregman. Para isto, o fecho da zona $\Omega$ da distância de Bregman deve ser igual ao conjunto viável, ou seja, $\bar{\Omega}=C$. Para este método é necessário também que a distância de Bregman seja coerciva na fronteira de $\Omega$.

Definição 3.5. Se $\left\{y^{i}\right\}_{i \in \mathbb{N}} \in \Omega$ e $y^{i} \longrightarrow y$, com y na fronteira de $\bar{\Omega}$, se para todo $x \in \Omega$ vale

$$
\left\langle\nabla h\left(y^{i}\right), x-y^{i}\right\rangle \rightarrow-\infty
$$

Neste caso, dizemos que a função de Bregman $h(\cdot)$ é coerciva na fronteira de $\Omega$.

O fato de uma distância de Bregman ser coerciva na fronteira de sua zona impede que uma projeção definida por esta distância seja um ponto na fronteira. No trabalho [10], Iusem mostra que isto se deve ao fato que na fronteira, o subdiferencial de uma distância de Bregman coerciva é vazio.

\section{Exemplos de distâncias de Bregman coercivas}

Nessa seção apresentamos alguns exemplos de distâncias de Bregman com a propriedade de coercividade.

- A zona é o interior de $\mathbb{R}_{+}^{n}$.

Um exemplo de função de Bregman é baseada em $h(x)=\sum_{i=1}^{n} x_{i} \ln \left(x_{i}\right)$, convencionando que $0 \ln (0)=0$. A distância de Bregman associada, conhecida como distância Kullback-Leibler, é:

$$
D_{h}(x, y)=\sum_{i=1}^{n}\left(x_{i} \ln \left(\frac{x_{i}}{y_{i}}\right)-x_{i}+y_{i}\right)
$$

- A zona é o interior de uma caixa do $\mathbb{R}^{n}$, isto é:

$$
\Omega=\left(a_{1}, b_{1}\right) \times \ldots \times\left(a_{n}, b_{n}\right)
$$

onde $a_{i}<b_{i}$ para $1 \leq i \leq n$.

Um exemplo de função de Bregman é:

$$
h(x)=\sum_{i=1}^{n}\left(\left(x_{i}-a_{i}\right) \ln \left(x_{i}-a_{i}\right)+\left(b_{i}-x_{i}\right) \ln \left(b_{i}-x_{i}\right)\right) .
$$


E a distância de Bregman associada é:

$$
D_{h}(x, y)=\sum_{i=1}^{n}\left(\left(x_{i}-a_{i}\right) \ln \frac{x_{i}-a_{i}}{y_{i}-a_{i}}+\left(b_{i}-x_{i}\right) \ln \frac{b_{i}-x_{i}}{b_{i}-y_{i}}\right) .
$$

Mais detalhes sobre estas funções podem ser encontrados nos trabalhos de Auslender, Teboulle e Silva em [2, 16].

A norma euclidiana ao quadrado é um exemplo de distância de Bregman que satisfaz a identidade dos três pontos, entretanto sua zona é o espaço $\mathbb{R}^{n}$. Sendo assim, usar esta distância no método de Auslender e Teboulle só faz sentido se queremos resolver um problema irrestrito. Este fato é lamentável, uma vez que gostaríamos de usar essa distância devido a suas propriedades e simplicidade.

\subsubsection{Construção da $\left\{\phi_{k}(\cdot)\right\}$ usando distâncias de Bregman coercivas}

Sejam $\gamma_{0}>0, x^{0} \in \operatorname{int}(C), \alpha_{k} \in(0,1), y^{k} \in \operatorname{int}(C), h(\cdot)$ uma função de Bregman coerciva na fronteira de $C$. Para todo $k \geq 0$ e para qualquer $x \in C$, a sequência $\left\{\phi_{k}(\cdot)\right\}$ é definida recursivamente da seguinte forma:

$$
\begin{aligned}
\phi_{0}(x) & =f\left(x^{0}\right)+\gamma_{0} D_{h}\left(x, x^{0}\right), \\
\phi_{k+1}(x) & =\left(1-\alpha_{k}\right) \phi_{k}(x)+\alpha_{k} \ell_{k}\left(x, y^{k}\right), \\
\ell_{k}\left(x, y^{k}\right) & =f\left(y^{k}\right)+\left\langle\nabla f\left(y^{k}\right), x-y^{k}\right\rangle .
\end{aligned}
$$

Observe que, a menos da hipótese da função $h(\cdot)$ ser coerciva na fronteira de sua zona, a construção da sequência $\left\{\phi_{k}(\cdot)\right\}$ é idêntica a construção feita por Nesterov para o problema sem restrições quando tomamos $h(x)=\frac{\|x\|^{2}}{2}$. Entretanto, em relação ao método restrito descrito na seção anterior, a diferença fundamental é que naquele método a aproximação linear é feita em relação ao ponto $x^{k+1}$ e o minimizador de $\phi_{k+1}(\cdot)$ depende de $x^{k+1}$.

Lema 3.6. A sequência $\left\{\phi_{k}(\cdot)\right\}$ definida por (3.10)-(3.12) satisfaz para todo $x \in C$

$$
\phi_{k+1}(x)-f(x) \leq\left(1-\alpha_{k}\right)\left(\phi_{k}(x)-f(x)\right)
$$

Demonstração: 
Pela equação (3.11),

$$
\phi_{k+1}(x)=\left(1-\alpha_{k}\right) \phi_{k}(x)+\alpha_{k} \ell_{k}\left(x, y^{k}\right) .
$$

Mas como $f(\cdot)$ é convexa em $C$, para todo $x \in C$ tem-se $f(x) \geq \ell_{k}\left(x, y^{k}\right)$. Logo,

$$
\phi_{k+1}(x) \leq\left(1-\alpha_{k}\right) \phi_{k}(x)+\alpha_{k} f(x) .
$$

Desta forma, para todo $x \in C$,

$$
\phi_{k+1}(x)-f(x) \leq\left(1-\alpha_{k}\right) \phi_{k}(x)-\left(1-\alpha_{k}\right) f(x)
$$

De onde segue a equação (3.13).

O próximo lema nos mostra que as funções da sequência $\left\{\phi_{k}(\cdot)\right\}$ admitem uma forma simples.

Lema 3.7. Sejam $\gamma_{0}>0, v^{0}=x^{0} \in \operatorname{int}(C)$ e $\phi_{0}^{*}=f\left(x^{0}\right)$. Então, para toda sequência $\left\{y^{k}\right\} \subset C$, as funções $\phi_{k}(\cdot)$ definidas por (3.10),(3.11), (3.12) tem a forma

$$
\phi_{k}(x)=\phi_{k}^{*}+\gamma_{k} D_{h}\left(x, v^{k}\right)
$$

em que, $v^{k}=\operatorname{argmin}\left\{\phi_{k}(x) \mid x \in C\right\}$ e $\phi_{k}^{*}=\phi_{k}\left(v^{k}\right)$.

Demonstração:

Da hipótese que $v^{0}=x^{0}$ e tomando $\phi_{0}^{*}=f\left(x^{0}\right)$ segue que para todo $x \in C$, $\phi_{0}(x)=\phi_{0}^{*}+\gamma_{0} D_{h}\left(x, v^{0}\right)$. Vamos supor, por hipótese de indução, que para algum $k \geq 0$ e todo $x \in C, \phi_{k}(x)=\phi_{k}^{*}+\gamma_{k} D_{h}\left(x, v^{k}\right)$. Iremos provar que $\phi_{k+1}(x)=$ $\phi_{k+1}^{*}+\gamma_{k+1} D_{h}\left(x, v^{k+1}\right)$.

De (3.11)

$$
\phi_{k+1}(x)=\left(1-\alpha_{k}\right) \phi_{k}(x)+\alpha_{k} \ell_{k}\left(x, y^{k}\right) .
$$

Da hipótese de indução e fazendo $\gamma_{k+1}=\left(1-\alpha_{k}\right) \gamma_{k}$, segue que para todo $x \in C$

$$
\phi_{k+1}(x)=\left(1-\alpha_{k}\right) \phi_{k}^{*}+\gamma_{k+1} D_{h}\left(x, v^{k}\right)+\alpha_{k} \ell_{k}\left(x, y^{k}\right) .
$$

Como $v^{k+1}=\operatorname{argmin}\left\{\phi_{k+1}(x) \mid x \in C\right\}$, para todo $x \in C$,

$$
\phi_{k+1}^{*}=\left(1-\alpha_{k}\right) \phi_{k}^{*}+\gamma_{k+1} D_{h}\left(v^{k+1}, v^{k}\right)+\alpha_{k} \ell_{k}\left(v^{k+1}, y^{k}\right) .
$$


Subtraindo (3.16) de (3.15) temos para todo $x \in C$

$$
\phi_{k+1}(x)=\phi_{k+1}^{*}+\gamma_{k+1}\left[D_{h}\left(x, v^{k}\right)-D_{h}\left(v^{k+1}, v^{k}\right)\right]+\alpha_{k}\left[\ell_{k}\left(x, y^{k}\right)-\ell_{k}\left(v^{k+1}, y^{k}\right)\right]
$$

Logo,

$$
\left.\phi_{k+1}(x)=\phi_{k+1}^{*}+\gamma_{k+1}\left[D_{h}\left(x, v^{k}\right)-D_{h}\left(v^{k+1}, v^{k}\right)\right]+\alpha_{k}\left\langle\nabla f\left(y^{k}\right), x-v^{k+1}\right)\right\rangle .
$$

Agora, como $v^{k+1}=\operatorname{argmin}\left\{\phi_{k+1}(x) \mid x \in C\right\}$ deve satisfazer as condições de otimalidade da equação $(3.15)$ e do fato que $D_{h}\left(\cdot, v^{k}\right)$ é uma função de Bregman coerciva na fronteira de $C$, o minimizador de $\phi_{k+1}(\cdot)$ é único e está no interior do conjunto $C$. Desta forma, $v^{k+1}$ deve satisfazer a equação $\nabla \phi_{k+1}\left(v^{k+1}\right)=0$. Logo da equação (3.15) segue que

$$
\gamma_{k+1} \nabla_{1} D_{h}\left(v^{k+1}, v^{k}\right)+\alpha_{k} \nabla f\left(y_{k}\right)=0 .
$$

Em particular, podemos dizer que para todo $x \in C$,

$$
\left\langle\gamma_{k+1} \nabla_{1} D_{h}\left(v^{k+1}, v^{k}\right)+\alpha_{k} \nabla f\left(y_{k}\right), x-v^{k+1}\right\rangle=0 .
$$

Logo, para todo $x \in C$

$$
\phi_{k+1}(x)=\phi_{k+1}^{*}+\gamma_{k+1}\left[D_{h}\left(x, v^{k}\right)-D_{h}\left(v^{k+1}, v^{k}\right)\right]-\gamma_{k+1}\left\langle\nabla_{1} D_{h}\left(v^{k+1}, v^{k}\right), x-v^{k+1}\right\rangle .
$$

Como $D_{h}(\cdot, \cdot)$ satisfaz a identidade dos três pontos podemos escrever, para todo $x \in C$

$$
\left.D_{h}\left(x, v^{k}\right)=D_{h}\left(x, v^{k+1}\right)+D_{h}\left(v^{k+1}, v^{k}\right)+\left\langle\nabla_{1} D_{h}\left(v^{k+1}, v^{k}\right), x-v^{k+1}\right)\right\rangle .
$$

Portanto, para todo $x \in C$,

$$
\phi_{k+1}(x)=\phi_{k+1}^{*}+\gamma_{k+1} D_{h}\left(x, v^{k+1}\right) .
$$

No próximo lema mostramos como calcular o minimizar de $\phi_{k+1}(\cdot)$.

Lema 3.8. Se $v^{k+1}$ é o minimizador de $\phi_{k+1}(\cdot)$ no conjunto $C$, então $v^{k+1}$ satisfaz 
a seguinte equação:

$$
v^{k+1}=\operatorname{argmin}\left\{\left\langle x, \frac{\alpha_{k}}{\gamma_{k+1}} \nabla f\left(y^{k}\right)\right\rangle+D_{h}\left(x, v^{k}\right)\right\} .
$$

Demonstração:

Por hipótese, temos que $v^{k+1}$ minimiza $\phi_{k+1}(\cdot)$ em C. Então, pelos mesmos argumentos usados na demonstração do lema 3.7, vale a desigualdade (3.17). De onde decorre que

$$
\nabla_{1} D_{h}\left(v^{k+1}, v^{k}\right)+\frac{\alpha_{k}}{\gamma_{k+1}} \nabla f\left(y_{k}\right)=0
$$

Que é a condição de otimalidade de $v^{k+1}$ para (3.18).

Considerando que $\alpha^{k}$ é a maior raiz da equação $L \alpha_{k}^{2}=\left(1-\alpha_{k}\right) \gamma_{k}$, precisamos agora, mostrar como são escolhidos os pontos $x^{k}$ e $y^{k}$ de modo a garantir que em todas as iterações $\phi_{k+1}^{*} \geq f\left(x^{k+1}\right)$.

- $y^{k}$ é obtido através de um passo $\alpha$ na direção de $x^{k}$ para $v^{k}$

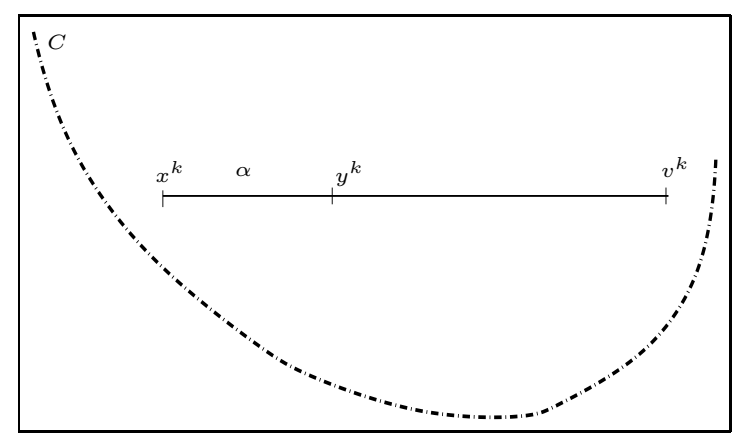

Figura 3.2: Escolha de $y^{k}$

- $x^{k+1}$ é obtido através de um passo $\alpha$ na direção de $x^{k}$ para $v^{k+1}$

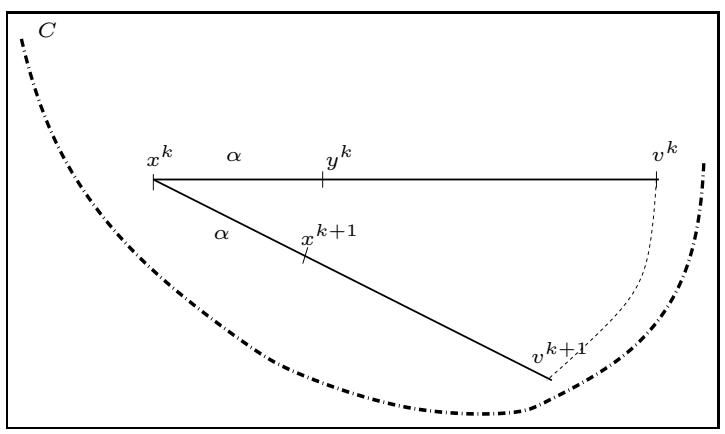

Figura 3.3: Escolha de $x^{k+1}$ 
No próximo teorema mostramos que estas escolhas garantem que $\phi_{k+1}^{*} \geq$ $f\left(x^{k+1}\right)$ para todo $k \geq 0$.

Teorema 3.9. Seja $L>0$ e suponha que $D_{h}(\cdot, \cdot)$ seja fortemente convexa com parâmetro de convexidade $\sigma>0$. Suponha também, que a iteração $k \geq 0$ começa com $\alpha_{k} \in(0,1)$ sendo a maior raiz da equação $L \alpha_{k}^{2}=\sigma\left(1-\alpha_{k}\right) \gamma_{k}, v^{k} \in \operatorname{int}(C)$, $x^{k} \in C$ tal que $\phi_{k}^{*} \geq f\left(x^{k}\right)$. Se as seguintes escolhas são feitas

$$
y^{k}=x^{k}+\alpha_{k}\left(v^{k}-x^{k}\right)
$$

$v^{k+1}$ é dado pelo lema 3.8,

$$
x^{k+1}=x^{k}+\alpha_{k}\left(v^{k+1}-x^{k}\right),
$$

e $\gamma_{k+1}=\left(1-\alpha_{k}\right) \gamma_{k}$, então a iteração termina com

$$
\phi_{k+1}^{*} \geq f\left(x^{k+1}\right)
$$

Demonstração:

Das hipóteses que $\phi_{k}(x)=\phi_{k}^{*}+\gamma_{k} D_{h}\left(x, v^{k}\right)$ e $\gamma_{k+1}=\left(1-\alpha_{k}\right) \gamma_{k}$ podemos garantir que vale a equação (3.16), ou seja,

$$
\phi_{k+1}^{*}=\left(1-\alpha_{k}\right) \phi_{k}^{*}+\gamma_{k+1} D_{h}\left(v^{k+1}, v^{k}\right)+\alpha_{k} \ell_{k}\left(v^{k+1}, y^{k}\right) .
$$

Da hipótese que $\phi_{k}^{*} \geq f\left(x^{k}\right)$ e da convexidade da $f(\cdot)$ podemos escrever

$$
\phi_{k}^{*} \geq f\left(x^{k}\right) \geq f\left(y^{k}\right)+\left\langle\nabla f\left(y^{k}\right), x^{k}-y^{k}\right\rangle .
$$

Assim,

$$
\begin{aligned}
\phi_{k+1}^{*} \geq & \left(1-\alpha_{k}\right)\left[f\left(y^{k}\right)+\left\langle\nabla f\left(y^{k}\right), x^{k}-y^{k}\right\rangle\right]+\gamma_{k+1} D_{h}\left(v^{k+1}, v^{k}\right) \\
& +\alpha_{k}\left[f\left(y^{k}\right)+\left\langle\nabla f\left(y^{k}\right), v^{k+1}-y^{k}\right\rangle\right],
\end{aligned}
$$

de onde decorre que

$$
\begin{aligned}
\phi_{k+1}^{*} \geq & f\left(y^{k}\right)+\gamma_{k+1} D_{h}\left(v^{k+1}, v^{k}\right) \\
& +\left\langle\nabla f\left(y^{k}\right),\left(1-\alpha_{k}\right)\left(x^{k}-y^{k}\right)+\alpha_{k}\left(v^{k+1}-y^{k}\right)\right\rangle .
\end{aligned}
$$


Como $y^{k}$ satisfaz a equação (3.19) podemos escrever que

$$
\alpha_{k} v^{k}+\left(1-\alpha_{k}\right) x^{k}-y^{k}=0
$$

Logo,

$$
\alpha_{k} v^{k}+\left(1-\alpha_{k}\right) x^{k}-y^{k}+\alpha_{k} v^{k+1}=\alpha_{k} v^{k+1} .
$$

Desta forma,

$$
\begin{aligned}
\alpha_{k}\left(v^{k+1}-v^{k}\right) & =\left(1-\alpha_{k}\right) x^{k}-y^{k}+\alpha_{k} v^{k+1} \\
& =\left(1-\alpha_{k}\right) x^{k}-y^{k}+\alpha_{k} v^{k+1}+\alpha_{k}\left(y^{k}-y^{k}\right) \\
& =\left(1-\alpha_{k}\right) x^{k}-\left(1-\alpha_{k}\right) y^{k}+\alpha_{k}\left(v^{k+1}-y^{k}\right) \\
& =\left(1-\alpha_{k}\right)\left(x^{k}-y^{k}\right)+\alpha_{k}\left(v^{k+1}-y^{k}\right) .
\end{aligned}
$$

Então, substituindo na equação (3.21), temos que

$$
\phi_{k+1}^{*} \geq f\left(y^{k}\right)+\gamma_{k+1} D_{h}\left(v^{k+1}, v^{k}\right)+\left\langle\nabla f\left(y^{k}\right), \alpha_{k}\left(v^{k+1}-v^{k}\right)\right\rangle .
$$

Subtraindo (3.19) de (3.20), temos $\alpha_{k}\left(v^{k+1}-v^{k}\right)=x^{k+1}-y^{k}$. Ainda, da hipótese de $D_{h}(\cdot, \cdot)$ ser fortemente convexa com parâmetro $\sigma>0$, garante-se

$$
D_{h}\left(v^{k+1}, v^{k}\right) \geq \frac{\sigma}{2}\left\|v^{k+1}-v^{k}\right\|^{2}=\frac{\sigma}{2 \alpha_{k}^{2}}\left\|x^{k+1}-y^{k}\right\|^{2}
$$

Juntando estes fatos obtemos a seguinte expressão

$$
\phi_{k+1}^{*} \geq f\left(y^{k}\right)+\frac{\gamma_{k+1} \sigma}{2 \alpha_{k}^{2}}\left\|x^{k+1}-y^{k}\right\|^{2}+\left\langle\nabla f\left(y^{k}\right), x^{k+1}-y^{k}\right\rangle .
$$

Como o gradiente de $f(\cdot)$ é Lipschitz contínuo, vale que

$$
f\left(y^{k}\right)+\left\langle\nabla f\left(y^{k}\right), x^{k+1}-y^{k}\right\rangle+\frac{L}{2}\left\|x^{k+1}-y^{k}\right\|^{2} \geq f\left(x^{k+1}\right) .
$$

Assim,

$$
\phi_{k+1}^{*} \geq f\left(x^{k+1}\right)+\left(\frac{\sigma \gamma_{k+1}}{2 \alpha_{k}^{2}}-\frac{L}{2}\right)\left\|x^{k+1}-y^{k}\right\|^{2} .
$$

Portanto, da escolha de $\alpha_{k}$ concluímos que,

$$
\phi_{k+1}^{*} \geq f\left(x^{k+1}\right)
$$

A demonstração que $\alpha_{k} \in(0,1)$ é idêntica a demonstração feita no teorema 2.8 . 
Este resultado mostra explicitamente como escolher $y^{k}, x^{k}$ e $\alpha_{k}$ de modo a garantir (3.5) e permite sintetizar o método do modo que apresentamos abaixo.

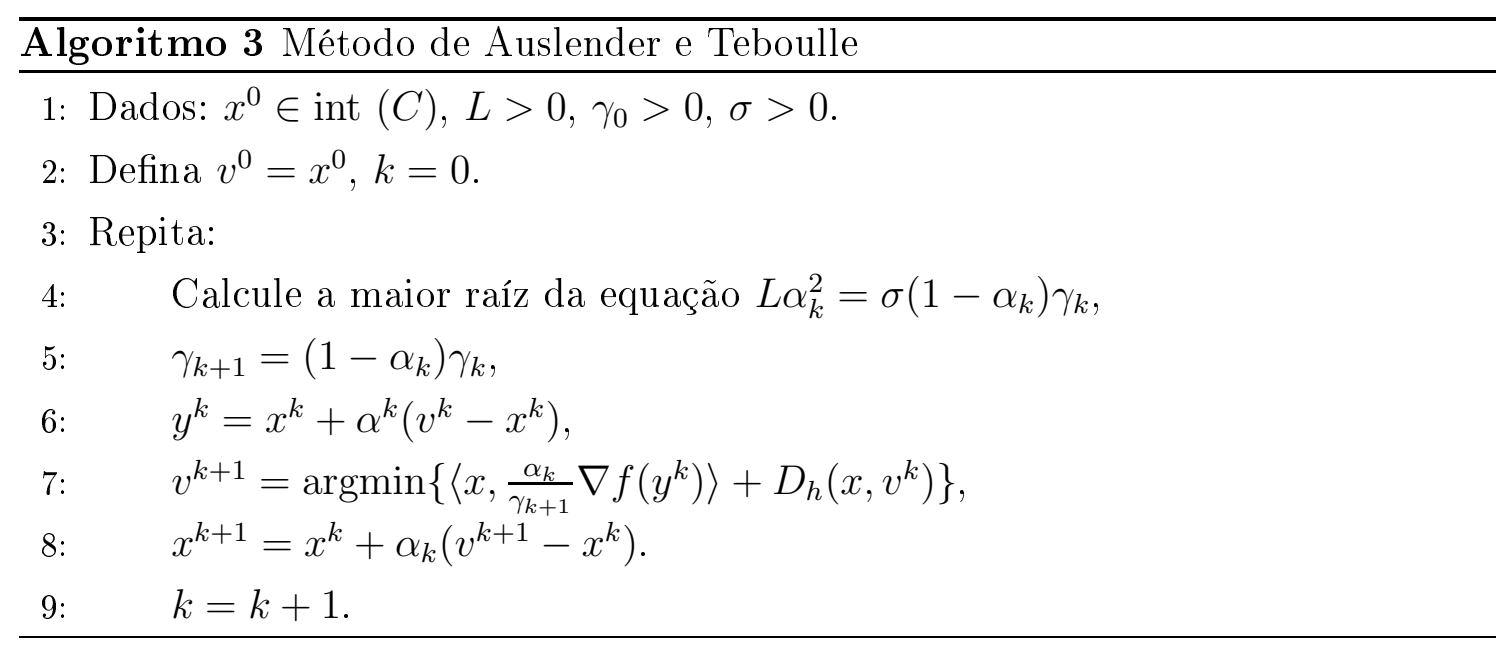

No início desta seção discutimos que é preciso garantir (3.4) e (3.5) para que a sequência $\left\{x^{k}\right\}$ satisfaça a desigualdade (3.3). O lema 3.6 e o teorema 3.9 mostram exatamente como garantir (3.4) e (3.5). Nos próximos resultados mostramos que a escolha da sequência $\left\{\alpha_{k}\right\}$ garante também que este método é ótimo.

Lema 3.10. Sejam $\gamma_{k}>0$ e $\alpha_{k} \in(0,1)$ definidos por $L \alpha_{k}^{2}=\sigma\left(1-\alpha_{k}\right) \gamma_{k}$ com $\gamma_{k+1}=\left(1-\alpha_{k}\right) \gamma_{k}$. Defina $\lambda_{k}:=\prod_{i=0}^{k-1}\left(1-\alpha_{i}\right)$, então

$$
\lambda_{k} \leq \frac{4 L}{\left(2 \sqrt{L}+k \sqrt{\sigma \gamma_{0}}\right)^{2}}
$$

A demonstração deste lema pode ser vista em [2, 15].

Teorema 3.11. Sejam $\left\{x^{k}\right\},\left\{y^{k}\right\}$ e $\left\{\alpha_{k}\right\}$ as sequências geradas pelo método de Auslender e Teboulle e seja $x^{*}$ a solução ótima do problema 3.1. Então para qualquer $k \geq 0$ temos

$$
f\left(x^{k}\right)-f\left(x^{*}\right) \leq \frac{4 L}{\sigma \gamma_{0} k^{2}} C\left(x^{*}, x^{0}\right)=O\left(\frac{1}{k^{2}}\right)
$$

em que $C\left(x^{*}, x^{0}\right)=f\left(x^{0}\right)+\gamma_{0} D_{h}\left(x^{*}, x^{0}\right)-f\left(x^{*}\right)$. Em particular, a sequência $\left\{x^{k}\right\}$ é minimizante, isto é, $f\left(x^{k}\right) \rightarrow f\left(x^{*}\right)$ e é viável. 
Demonstração:

Dos teoremas 3.6 e 3.9 temos que as condições (3.4) e (3.5) são satisfeitas e desta forma a sequência $\left\{x^{k}\right\}$ satisfaz a seguinte desigualdade,

$$
f\left(x^{k}\right)-f\left(x^{*}\right) \leq \lambda_{k}\left(\phi_{0}\left(x^{*}\right)-f\left(x^{*}\right)\right) .
$$

Como $\phi_{0}\left(x^{*}\right)=f\left(x^{0}\right)+\gamma_{0} D_{h}\left(x^{*}, x^{0}\right)$, podemos escrever a seguinte expressão

$$
f\left(x^{k}\right)-f\left(x^{*}\right) \leq \lambda_{k} C\left(x^{*}, x^{0}\right)
$$

Pelo lema 3.10 obtemos

$$
\lambda_{k} \leq \frac{4 L}{\left(2 \sqrt{L}+k \sqrt{\sigma \gamma_{0}}\right)^{2}} \leq \frac{4 L}{\sigma \gamma_{0} k^{2}}
$$

De onde ocorre (3.22). A sequência $\left\{x^{k}\right\}$ é viável, pois cada ponto dela é escolhido sendo uma combinação convexa entre dois pontos do conjunto viável e está no interior de $C$.

Do teorema 3.11 obtemos que

$$
f\left(x^{k}\right)-f\left(x^{*}\right)=O\left(\frac{1}{k^{2}}\right)
$$

Como foi exposto no capítulo introdutório, isto quer dizer que uma solução ótima $x^{*}$ pode ser estimada, com precisão $\epsilon$, em um número proporcional a $\frac{1}{\sqrt{\epsilon}}$ iterações, garantindo assim que o método é ótimo.

\subsection{Generalização para projeções não interiores}

O uso de distâncias de Bregman com a propriedade de coercividade na fronteira de sua zona descarta a possibilidade de usarmos a distância euclidiana. Isto porque, como a zona da distância euclidiana é todo o espaço $\mathbb{R}^{n}$, embora esta distância seja uma distância de Bregman, ela só poderia ser usada para minimizar em todo o $\mathbb{R}^{n}$. Porém, como esta distância já foi amplamente estudada, conhecemos muitas de suas propriedades. Por exemplo, podemos falar de distâncias e projeções em um 
sentido tradicional. Além disto, computacionalmente, sabemos que esta distância se comporta muito bem.

Nessa seção mostramos nosso primeiro resultado que estende o método da seção anterior, permitindo o uso da distância euclidiana ao quadrado como distância de Bregman em métodos restritos.

A diferença fundamental com o método anterior é que vamos definir distâncias de Bregman com zonas que podem conter propriamente o interior do conjunto viável. Como na seção 3.2.2, gostaríamos de construir uma sequência de funções $\left\{\phi_{k}(\cdot)\right\}$ de forma que cada função, que é obtida combinando a função anterior (que é simples) com uma aproximação inferior, continuasse sendo uma função simples. Nas próximas definições veremos como fazer esta construção a partir da construção de uma sequência de funções auxiliares que chamaremos $\left\{\tilde{\phi}_{k}(\cdot)\right\}$, mas antes faremos algumas hipóteses:

Hipótese 3.12. Consideramos $h(\cdot)$ uma função de Bregman como definido em 3.3 e sobre ela supomos ainda as seguintes hipóteses.

- o interior do conjunto viável $C$ está contido na zona de $h(\cdot)$, isto é, int $(C) \subset$ $\Omega$.

- $h(\cdot)$ é $\sigma$-fortemente convexa em $\Omega$;

- o subdiferencial de $h(\cdot)$ na fronteira de $\bar{\Omega}$ é vazio.

Definição 3.13. Sejam $y^{k} \in \mathbb{R}^{n}, v^{k} \in C, \gamma_{k}>0, \phi_{k}^{*} \in \mathbb{R}$ e $\alpha_{k} \in(0,1)$. Considere as seguintes funções:

1. $\phi_{k}(x)=\phi_{k}^{*}+\gamma_{k} D_{h}\left(x, v^{k}\right)$

2. $\ell\left(x, y^{k}\right)=f\left(y^{k}\right)+\left\langle\nabla f\left(y^{k}\right), x-y^{k}\right\rangle$

Definimos:

$$
\tilde{\phi}_{k+1}(x)=\left(1-\alpha_{k}\right) \phi_{k}(x)+\alpha_{k} \ell\left(x, y^{k}\right)
$$

No lema 3.16 abaixo, mostramos que as funções da sequência $\left\{\tilde{\phi}_{k}(\cdot)\right\}$ não são funções simples, porém qualquer função desta sequência sempre majora uma função simples. Sendo assim, precisamos buscar uma sequência de funções simples majorada pela sequência $\left\{\tilde{\phi}_{k}(\cdot)\right\}$. 
Definição 3.14. Definimos

$$
\begin{aligned}
\bar{v} & =\operatorname{argmin}\left\{\tilde{\phi}_{k+1}(x) \mid x \in \mathbb{R}^{n}\right\}, \\
v^{+} & =\operatorname{argmin}\left\{D_{h}(x, \bar{v}) \mid x \in C\right\}, \\
\tilde{\phi}_{k+1}^{*} & =\tilde{\phi}_{k+1}\left(v^{+}\right), \\
\gamma_{+} & =\left(1-\alpha_{k}\right) \gamma_{k} .
\end{aligned}
$$

Lema 3.15. $\bar{v}$ e $v^{+}$estão bem definidos.

Demonstração:

Como a função $h(\cdot)$ é fortemente convexa, $\tilde{\phi}_{k}(\cdot)$ é uma combinação convexa de uma função fortemente convexa com uma função linear, desta forma possui um único minimizador e portanto $\bar{v}$ está bem definido. Ainda, do fato que $h(\cdot)$ é fortemente convexa e $D_{h}(\cdot, \cdot)$ é induzida por $h(\cdot)$ segue que $v^{+}$está bem definido.

Lema 3.16. Considere a definição 3.13, com $h(\cdot)$ satisfazendo as hipóteses 3.12, e os pontos descritos na definição 3.14. Então para todo $x \in C$,

$$
\tilde{\phi}_{k+1}(x) \geq \tilde{\phi}_{k+1}^{*}+\gamma_{+} D_{h}\left(x, v^{+}\right) .
$$

Demonstração:

Relembrando a definição 3.13 , temos que $\tilde{\phi}_{k+1}(x)=\left(1-\alpha_{k}\right) \phi_{k}(x)+\alpha_{k} \ell\left(x, y^{k}\right)$. Logo, das hipóteses, podemos reescrever que para todo $x \in C$,

$$
\tilde{\phi}_{k+1}(x)=\left(1-\alpha_{k}\right) \phi_{k}^{*}+\gamma_{+} D_{h}\left(x, v^{k}\right)+\alpha_{k} \ell\left(x, y^{k}\right),
$$

Em particular,

$$
\tilde{\phi}_{k+1}^{*}=\left(1-\alpha_{k}\right) \phi_{k}^{*}+\gamma_{+} D_{h}\left(v^{+}, v^{k}\right)+\alpha_{k} \ell\left(v^{+}, y^{k}\right) .
$$

Subtraindo (3.31) de (3.30) temos para todo $x \in C$,

$$
\tilde{\phi}_{k+1}(x)=\tilde{\phi}_{k+1}^{*}+\gamma_{+}\left[D_{h}\left(x, v^{k}\right)-D_{h}\left(v^{+}, v^{k}\right)\right]+\alpha_{k}\left\langle\nabla f\left(y^{k}\right), x-v^{+}\right\rangle .
$$

Como $\bar{v}=\operatorname{argmin}\left\{\tilde{\phi}_{k+1}(x) \mid x \in \mathbb{R}^{n}\right\}$,

$$
\gamma_{+} \nabla_{1} D_{h}\left(\bar{v}, v^{k}\right)+\alpha_{k} \nabla f\left(y^{k}\right)=\nabla \tilde{\phi}_{k+1}(\bar{v})=0
$$

ou seja, $\alpha_{k} \nabla f\left(y^{k}\right)=-\gamma_{+} \nabla_{1} D_{h}\left(\bar{v}, v^{k}\right)$. Substituindo esta última expressão em (3.32), 
temos que

$$
\tilde{\phi}_{k+1}(x)=\tilde{\phi}_{k+1}^{*}+\gamma_{+}\left[D_{h}\left(x, v^{k}\right)-D_{h}\left(v^{+}, v^{k}\right)-\left\langle\nabla_{1} D_{h}\left(\bar{v}, v^{k}\right), x-v^{+}\right\rangle\right]
$$

Ainda, como $v^{+}=\operatorname{argmin}\left\{D_{h}(x, \bar{v}) \mid x \in C\right\}$, as seguintes desigualdades são satisfeitas para todo $x \in C$,

$$
\begin{aligned}
0 & \leq\left\langle\nabla_{1} D_{h}\left(v^{+}, \bar{v}\right), x-v^{+}\right\rangle \\
& =\left\langle\nabla h\left(v^{+}\right)-\nabla h(\bar{v}), x-v^{+}\right\rangle \\
& =\left\langle\nabla h\left(v^{+}\right)-\nabla h\left(v^{k}\right)+\nabla h\left(v^{k}\right)-\nabla h(\bar{v}), x-v^{+}\right\rangle .
\end{aligned}
$$

Esta última desigualdade pode ser reescrita da seguinte maneira,

$$
\left\langle\nabla h\left(v^{+}\right)-\nabla h\left(v^{k}\right), x-v^{+}\right\rangle \geq\left\langle\nabla h(\bar{v})-\nabla h\left(v^{k}\right), x-v^{+}\right\rangle
$$

e da expressão da derivada de $D_{h}(\cdot, \cdot)$ em relação a primeira variável, temos que

$$
\left\langle\nabla_{1} D_{h}\left(v^{+}, v^{k}\right), x-v^{+}\right\rangle \geq\left\langle\nabla_{1} D_{h}\left(\bar{v}, v^{k}\right), x-v^{+}\right\rangle .
$$

Substituindo esta expressão em (3.33), obtemos que

$$
\tilde{\phi}_{k+1}(x) \geq \tilde{\phi}_{k+1}^{*}+\gamma_{+}\left[D_{h}\left(x, v^{k}\right)-D_{h}\left(v^{+}, v^{k}\right)-\left\langle\nabla_{1} D_{h}\left(v^{+}, v^{k}\right), x-v^{+}\right\rangle\right] .
$$

Como a distância de Bregman satisfaz a identidade dos três pontos, concluímos que para todo $x \in C$,

$$
\tilde{\phi}_{k+1}(x) \geq \tilde{\phi}_{k+1}^{*}+\gamma_{+} D_{h}\left(x, v^{+}\right)
$$

Deste resultado temos que $\tilde{\phi}_{k+1}(\cdot)$ majora uma função simples. Podemos então escolher a função $\phi_{k+1}(\cdot)$ como a função majorada por $\tilde{\phi}_{k+1}(\cdot)$ dada pela lema anterior. Este fato é formalizado pela seguinte definição:

Definição 3.17. Definimos:

$$
\begin{aligned}
\gamma_{k+1} & =\gamma_{+} \\
v^{k+1} & =v^{+} \\
\phi_{k+1}^{*} & =\tilde{\phi}_{k+1}^{*} \\
\phi_{k+1}(x) & =\phi_{k+1}^{*}+\gamma_{k+1} D_{h}\left(x, v^{k+1}\right) .
\end{aligned}
$$


Logo para todo $x \in C$ temos que $\tilde{\phi}_{k+1}(x) \geq \phi_{k+1}(x)$. Desta forma nosso método irá trabalhar com duas sequências de funções e $\left\{\tilde{\phi}_{k+1}(\cdot)\right\}$ funcionará como uma sequência auxiliar para a construção da sequência $\left\{\phi_{k}(\cdot)\right\}$.

Lema 3.18. As sequências $\left\{\tilde{\phi}_{k}(\cdot)\right\}$ e $\left\{\phi_{k}(\cdot)\right\}$ acima satisfazem para todo $x \in C$,

$$
\tilde{\phi}_{k+1}(x)-f(x) \leq\left(1-\alpha_{k}\right)\left(\phi_{k}(x)-f(x)\right) .
$$

Demonstração:

De $(3.24)$

$$
\tilde{\phi}_{k+1}(x)=\left(1-\alpha_{k}\right) \phi_{k}(x)+\alpha_{k} \ell\left(x, y^{k}\right),
$$

e da convexidade da $f(\cdot)$ segue que $f(x) \geq \ell_{k}\left(x, y^{k}\right)$ para todo $x \in C$. Logo

$$
\tilde{\phi}_{k+1}(x) \leq\left(1-\alpha_{k}\right) \phi_{k}(x)+\alpha_{k} f(x)+(f(x)-f(x)) .
$$

Desta forma, para todo $x \in C$,

$$
\tilde{\phi}_{k+1}(x)-f(x) \leq\left(1-\alpha_{k}\right) \phi_{k}(x)-\left(1-\alpha_{k}\right) f(x),
$$

Ou seja,

$$
\tilde{\phi}_{k+1}(x)-f(x) \leq\left(1-\alpha_{k}\right)\left(\phi_{k}(x)-f(x)\right) .
$$

Lema 3.19. Se $\gamma_{0}=1, x^{*}$ é a solução do problema (3.1) e a sequência $\left\{\phi_{k}(\cdot)\right\}$ dada na definição 3.17 satisfaz $\phi_{k+1}^{*} \geq f\left(x^{k+1}\right)$ para todo $k \geq 0$, então podemos garantir que

$$
f\left(x^{k+1}\right)-f\left(x^{*}\right) \leq \lambda_{k+1}\left(\phi_{0}\left(x^{*}\right)-f\left(x^{*}\right)\right) .
$$

Demonstração:

Pelo lema 3.16 para todo $x \in C, \tilde{\phi}_{k+1}(x) \geq \phi_{k+1}(x)$. Logo, para todo $x \in C$

$$
\phi_{k+1}(x)-f(x) \leq \tilde{\phi}_{k+1}(x)-f(x) .
$$

E o lema 3.18 garante que para todo $x \in C$,

$$
\tilde{\phi}_{k+1}(x)-f(x) \leq\left(1-\alpha_{k}\right)\left(\phi_{k}(x)-f(x)\right) .
$$


Juntando este dois fatos podemos escrever que para todo $x \in C$

$$
\phi_{k+1}(x)-f(x) \leq\left(1-\alpha_{k}\right)\left(\phi_{k}(x)-f(x)\right)
$$

Prosseguindo com os mesmos argumentos chegamos que para todo $x \in C$

$$
\phi_{k+1}(x)-f(x) \leq \prod_{i=0}^{k}\left(1-\alpha_{i}\right) \gamma_{0}\left(\phi_{0}(x)-f(x)\right) \text {. }
$$

Como $\gamma_{0}=1$ e $\lambda_{k+1}=\prod_{i=0}^{k}\left(1-\alpha_{i}\right)$, tomando $x=x^{*}$ podemos escrever

$$
\phi_{k+1}\left(x^{*}\right)-f\left(x^{*}\right) \leq \lambda_{k+1}\left(\phi_{0}\left(x^{*}\right)-f\left(x^{*}\right)\right)
$$

Usando a hipótese que $f\left(x^{k+1}\right) \leq \phi_{k+1}^{*}$ e o fato que $\phi_{k+1}^{*} \leq \phi_{k+1}\left(x^{*}\right)$ concluímos que

$$
f\left(x^{k+1}\right)-f\left(x^{*}\right) \leq \lambda_{k+1}\left(\phi_{0}\left(x^{*}\right)-f\left(x^{*}\right)\right)
$$

Desse lema temos que, as sequências $\left\{\phi_{k}(\cdot)\right\}$ e $\left\{x^{k}\right\}$ construídas nessa seção garantem a desigualdade (3.3). Sendo assim, poderíamos aqui enunciar um novo método, idêntico ao método de Auslender e Teboulle, exceto pela troca da distância de Bregman com zona igual ao conjunto viável por uma distância de Bregman cuja zona contém o conjunto viável, de maneira que a distância euclidiana ao quadrado pode ser usada.

Em [7], Gonzaga e Karas melhoraram a eficiência do método de Nesterov para problemas sem restrições. A forma como fizeram isso foi escolhendo novas sequências $\left\{y^{k}\right\}$ e $\left\{x^{k}\right\}$, de modo a aumentar o decréscimo da função objetivo. Inspirados neste trabalho, tentamos a seguir novas escolhas das sequências $\left\{y^{k}\right\}$ e $\left\{x^{k}\right\}$, com objetivo de aumentar a eficiência no novo método sugerido acima.

\subsubsection{Tentativa de aumentar o decréscimo da função objetivo}

Recordando o algoritmo 3, o ponto $y^{k}$ é escolhido como uma combinação convexa entre os pontos $x^{k}$ e $v^{k}$, definidos na iteração anterior e $x^{k+1}$ é uma combinação convexa de $x^{k}$ e o novo $v^{k+1}$. Em nenhum momento da construção destes pontos foi exigido algum decréscimo na função objetivo.

Como no trabalho de Gonzaga e Karas [7] observamos que, talvez, seja 
possível escolher um ponto $y^{k}$, entre $x^{k}$ e $v^{k}$, onde tenhamos alguma garantia do decréscimo da função objetivo e, além disto, com tamanho de passo maior que o tamanho do passo no método de Auslender e Teboulle. Ressaltando que, aumentar o tamanho do passo é potencialmente interessante, pois aumenta a velocidade de convergência do método, uma vez que a sequência dos valores $\lambda_{k}$ tendem a zero mais rapidamente. Para isso, fixada uma iteração $k$, procuramos um ponto $z$ entre $x^{k}$ e $v^{k}$ tal que $f(z) \leq f\left(x^{k}\right)$ e definimos $\beta=\frac{\left\|x^{k}-z\right\|}{\left\|v^{k}-x^{k}\right\|}$,

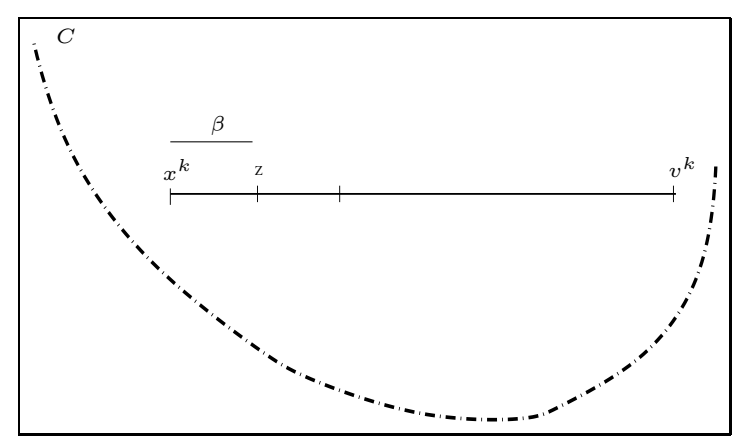

Figura 3.4: $f(z) \leq f\left(x^{k}\right)$

Denotamos por $\alpha_{A T}$ a maior raíz da equação $L \alpha_{A T}^{2}=\sigma \gamma_{k}\left(1-\alpha_{A T}\right)$. O ponto $y^{k}$ é escolhido a partir de $z$ como

$$
y^{k}=z+\alpha_{A T}\left(v^{k}-z\right) .
$$

Como $z=x^{k}+\beta\left(v^{k}-x^{k}\right)$, podemos escrever que

$$
\begin{aligned}
y^{k} & =z+\alpha_{A T}\left(v^{k}-z\right) \\
& =x^{k}+\beta\left(v^{k}-x^{k}\right)+\alpha_{A T}\left(v^{k}-x^{k}-\beta\left(v^{k}-x^{k}\right)\right) \\
& =x^{k}+\left(\beta+\alpha_{A T}(1-\beta)\right)\left(v^{k}-x^{k}\right) .
\end{aligned}
$$

Definindo $\theta=\beta+\alpha_{A T}(1-\beta)$, que é efetivamente maior ou igual a $\alpha_{A T}$, obtemos

$$
y^{k}=x^{k}+\theta\left(v^{k}-x^{k}\right) .
$$




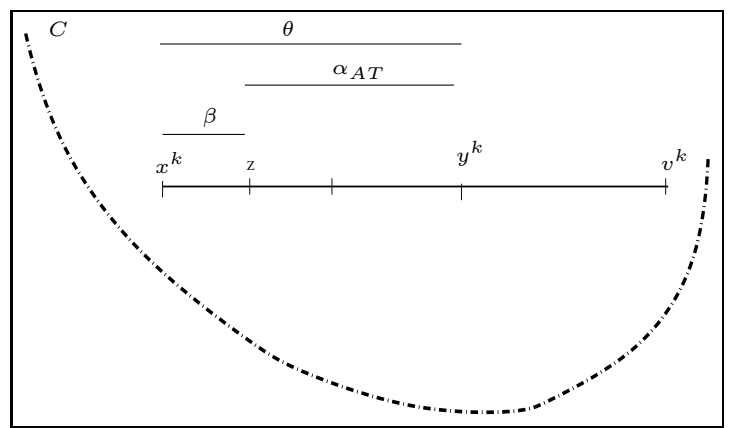

Figura 3.5: $\theta=\beta+\alpha_{A T}(1-\beta)$

De acordo com o que expomos na seção anterior, o método de Auslender e Teboulle gera uma sequência de pontos $\left\{x^{k}\right\}$ interiores ao conjunto viável. Com a extensão do método anterior com distâncias de Bregman definidas em conjuntos que contenham o conjunto viável, nosso objetivo foi construir uma sequência $\left\{x^{k}\right\}$ que pudesse obter uma maior decréscimo no valor da função objetivo. A maneira que fizemos isto foi tomando pontos projetados sobre o conjunto viável. No capítulo referente aos testes numéricos veremos que isto é fundamental para melhorar a eficiência do método. Porém, para melhorar a escolha de $x^{k+1}$, sem perder a otimalidade do método, não podemos permitir que o valor da função objetivo neste novo ponto cresça em relação ao ponto $x^{k+1}$, determinado por Auslender e Teboulle. Inspirados no trabalho de Gonzaga e Karas, nossa expectativa é, de que, forçar o decréscimo da função objetivo melhora a eficiência do método. Para isto, introduzimos um ponto intermediário $\hat{x}$, que era o ponto escolhido por Auslender e Teboulle. Este ponto pode ser reescrito da seguinte forma:

$$
\begin{aligned}
\hat{x} & =z+\alpha_{A T}\left(v^{k+1}-z\right) \\
& =\alpha_{A T} v^{k+1}+\left(1-\alpha_{A T}\right) z .
\end{aligned}
$$

Subtraindo (3.39) de (3.40) temos que:

$$
\hat{x}=y^{k}+\alpha_{A T}\left(v^{k+1}-v^{k}\right) .
$$




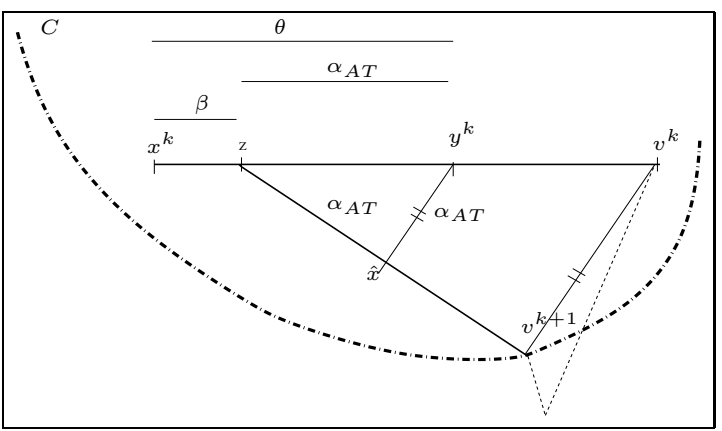

Figura 3.6: $\hat{x}=y^{k}+\alpha_{A T}\left(v^{k+1}-v^{k}\right)$

Em seguida, procuramos algum ponto onde o valor da função objetivo seja menor ou igual que o valor da função objetivo em $\hat{x}$. Este novo ponto chamamos de $x^{k+1}$. Caso não seja possível encontrar algum ponto onde o valor da função objetivo seja menor, ficamos com o ponto $\hat{x}$.

Na próxima seção mostramos um critério para estimar o valor da constante de Lipschitz do gradiente da função objetivo.

\subsection{2 $L$ adaptativo}

Como vimos no capítulo introdutório, o fato da função objetivo ter gradiente Lipschitz contínuo possibilita a construção de uma aproximação superior para $f(\cdot)$, cujo parâmetro de convexidade é o valor da constante de Lipschitz, que, de forma geral, é um parâmetro desconhecido.

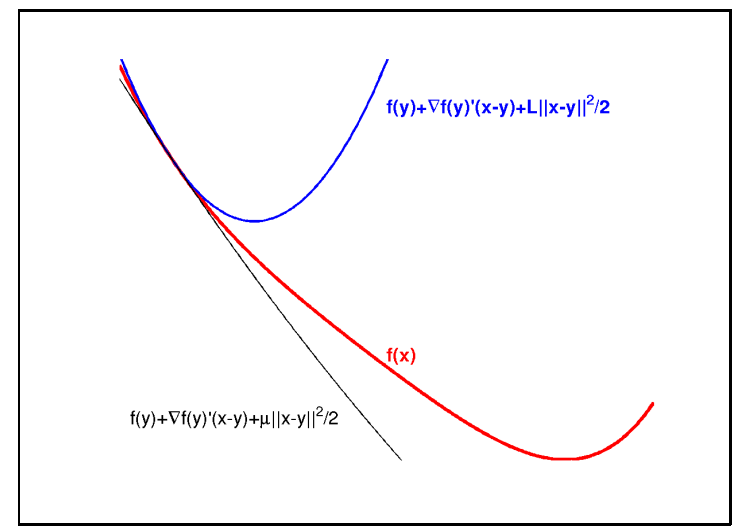

Figura 3.7: Aproximação superior

No teorema 3.9, é possível observar que a desigualdade fundamental, que aparece na demonstração, envolvendo a constante de Lipschitz do gradiente da função objetivo é 


$$
f(y)+\langle\nabla f(y), x-y\rangle+\frac{L}{2}\|x-y\|^{2} \geq f(x)
$$

que é uma aproximação superior da função $f(\cdot)$ no ponto $y$.

A pergunta que surge, naturalmente, é se, necessariamente, devemos usar a equação (3.42) com o valor verdadeiro de $L$. Na proposição abaixo, provamos que é possível construir outras aproximaçôes superiores e convexas para $f(\cdot)$ sempre que o parâmetro de convexidade seja uma estimativa superior do valor da constante de Lipschitz.

Proposição 3.20. Se $L^{a} \geq L$, então para qualquer $x, y \in C$

$$
f(y)+\langle\nabla f(y), x-y\rangle+\frac{L^{a}}{2}\|x-y\|^{2} \geq f(x) .
$$

Demonstração:

A demonstração segue diretamente da desigualdade (3.42).

Desta forma, qualquer valor $L^{a} \geq L$ garante que podemos construir uma aproximação superior da função $f(\cdot)$ da forma (3.43) e sempre que a aproximação seja superior podemos demonstrar um resultado equivalente ao teorema 3.9.

Agora, supondo que o método comece com um valor estimado para a constante de Lipschitz, este resultado fornece um critério para verificar se o valor estimado é maior ou não que o valor verdadeiro de $L$. Sabemos que, se o valor está acima de $L$ verdadeiro, então a desigualdade (3.42) é satisfeita. Se a desigualdade (3.42) não é satisfeita, dobramos o valor de $L$ e testamos novamente. Repetimos isto até encontrar $L^{a}$ que satisfaça a desigualdade (3.42). Para impedir que a nova iteração comece com um valor muito grande para o valor estimado de $L$, iniciamos esta iteração com o valor estimado sendo metade do valor de $L^{a}$, como Nesterov no algoritmo 2. Com isto, obtemos um método adaptativo para se obter uma estimativa para $L$.

Abaixo reescrevemos o teorema que garante que com nossas sugestões ainda é possível garantir que $\phi_{k+1}^{*} \geq f\left(x^{k+1}\right)$.

Teorema 3.21. Suponha que $D_{h}(\cdot, \cdot)$ é fortemente convexa com parâmetro $\sigma>0$ e que a iteração $k \geq 0$ começa com $\gamma_{k}>0, v^{k}, x^{k} \in \mathbb{R}^{n}$ tal que $\phi_{k}^{*} \geq f\left(x^{k}\right)$ e $\alpha_{k} \in(0,1)$ 
sendo a maior raiz da equação $L^{a} \alpha_{k}^{2}=\sigma\left(1-\alpha_{k}\right) \gamma_{k}$. Se $z=x_{k}+\beta\left(v^{k}-x^{k}\right)$ está no segmento $\left[x^{k}, v^{k}\right]$ tal que $f(z) \leq f\left(x^{k}\right)$ e as seguintes escolhas são feitas

$$
y^{k}=x^{k}+\theta\left(v^{k}-x^{k}\right), \operatorname{com} \theta=\beta+\alpha_{k}(1-\beta),
$$

$v^{k+1}$ é dado pelo lema 3.8,

$$
\hat{x}=y^{k}+\alpha_{k}\left(v^{k+1}-v^{k}\right),
$$

$\gamma_{k+1}=\left(1-\alpha_{k}\right) \gamma_{k}, L^{a}>0$ satisfaz a desigualdade (3.43) para $y=y^{k}$ e $x=\hat{x} e$ ainda $x^{k+1}$ satisfaz $f\left(x^{k+1}\right) \leq f(\hat{x})$. Então a iteração termina com

$$
\phi_{k+1}^{*} \geq f\left(x^{k+1}\right)
$$

Demonstração:

A demonstração deste teorema é uma adaptação da demonstração do teorema 3.9. Pela definição (3.13), para todo $x \in \mathbb{R}^{n}, \tilde{\phi}_{k+1}(x)=\left(1-\alpha_{k}\right) \phi_{k}(x)+\alpha_{k} \ell_{k}\left(x, y^{k}\right)$. Mas pela escolha de $\phi_{k}$, para todo $x \in C$,

$$
\tilde{\phi}_{k+1}(x)=\left(1-\alpha_{k}\right) \phi_{k}^{*}+\gamma_{k+1} D_{h}\left(x, v^{k}\right)+\alpha_{k} \ell_{k}\left(x, y^{k}\right) .
$$

Em particular, para $x=v_{k+1}$,

$$
\tilde{\phi}_{k+1}^{*}=\left(1-\alpha_{k}\right) \phi_{k}^{*}+\gamma_{k+1} D_{h}\left(v^{k+1}, v^{k}\right)+\alpha_{k} \ell_{k}\left(v^{k+1}, y^{k}\right) .
$$

Por hipótese temos que $\phi_{k}^{*} \geq f\left(x^{k}\right) \geq f(z)$ e da convexidade da $f(\cdot)$ podemos escrever:

$$
\phi_{k}^{*} \geq f(z) \geq f\left(y^{k}\right)+\left\langle\nabla f\left(y^{k}\right), z-y^{k}\right\rangle .
$$

Pela definição 3.17 temos que $\tilde{\phi}_{k+1}^{*}=\phi_{k+1}^{*}$, desta forma

$$
\begin{aligned}
\phi_{k+1}^{*} \geq & \left(1-\alpha_{k}\right)\left[f\left(y^{k}\right)+\left\langle\nabla f\left(y^{k}\right), z-y^{k}\right\rangle\right]+\gamma_{k+1} D_{h}\left(v^{k+1}, v^{k}\right) \\
& +\alpha_{k}\left[f\left(y^{k}\right)+\left\langle\nabla f\left(y^{k}\right), v^{k+1}-y^{k}\right\rangle\right] .
\end{aligned}
$$

Logo,

$$
\begin{aligned}
\phi_{k+1}^{*} & \geq f\left(y^{k}\right)+\gamma_{k+1} D_{h}\left(v^{k+1}, v^{k}\right) \\
& +\left\langle\nabla f\left(y^{k}\right),\left(1-\alpha_{k}\right)\left(z-y^{k}\right)+\alpha_{k}\left(v^{k+1}-y^{k}\right)\right\rangle .
\end{aligned}
$$


Definindo $\zeta=\left(1-\alpha_{k}\right)\left(z-y^{k}\right)+\alpha_{k}\left(v^{k+1}-y^{k}\right)$ temos

$$
\begin{aligned}
\zeta & =\left(1-\alpha_{k}\right)\left(z-y^{k}\right)+\alpha_{k}\left(v^{k+1}-y^{k}\right)+\alpha_{k} v^{k}-\alpha_{k} v^{k} \\
& =\left(1-\alpha_{k}\right)\left(z-y^{k}\right)+\alpha_{k}\left(v^{k}-y^{k}\right)+\alpha_{k}\left(v^{k+1}-v^{k}\right) .
\end{aligned}
$$

Como $z=x^{k}+\beta\left(v^{k}-x^{k}\right)$ as seguintes igualdades são satisfeitas

$$
\begin{aligned}
\zeta & =\left(1-\alpha_{k}\right)\left(x^{k}+\beta\left(v^{k}-x^{k}\right)-y^{k}\right)+\alpha_{k}\left(v^{k}-y^{k}\right)+\alpha_{k}\left(v^{k+1}-v^{k}\right) \\
& =\left(1-\alpha_{k}\right)\left(x^{k}+\beta\left(v^{k}-x^{k}\right)\right)+\alpha_{k} v^{k}-y^{k}+\alpha_{k}\left(v^{k+1}-v^{k}\right) \\
& =x^{k}-\alpha_{k} x^{k}+\left(1-\alpha_{k}\right) \beta\left(v^{k}-x^{k}\right)+\alpha_{k} v^{k}-y^{k}+\alpha_{k}\left(v^{k+1}-v^{k}\right) \\
& =x^{k}+\alpha_{k}\left(v^{k}-x^{k}\right)+\left(1-\alpha_{k}\right) \beta\left(v^{k}-x^{k}\right)-y^{k}+\alpha_{k}\left(v^{k+1}-v^{k}\right) \\
& =x^{k}+\left(\alpha_{k}+\left(1-\alpha_{k}\right) \beta\right)\left(v^{k}-x^{k}\right)-y^{k}+\alpha_{k}\left(v^{k+1}-v^{k}\right) \\
& =x^{k}+\left(\beta+(1-\beta) \alpha_{k}\right)\left(v^{k}-x^{k}\right)-y^{k}+\alpha_{k}\left(v^{k+1}-v^{k}\right) \\
& =x^{k}+\theta\left(v^{k}-x^{k}\right)-y^{k}+\alpha_{k}\left(v^{k+1}-v^{k}\right) .
\end{aligned}
$$

Logo da escolha de $y^{k}$ temos que

$$
\left(1-\alpha_{k}\right)\left(z-y^{k}\right)+\alpha_{k}\left(v^{k+1}-y^{k}\right)=\alpha_{k}\left(v^{k+1}-v^{k}\right) .
$$

Então substituindo esta equação em (3.47), segue que

$$
\phi_{k+1}^{*} \geq f\left(y^{k}\right)+\gamma_{k+1} D_{h}\left(v^{k+1}, v^{k}\right)+\left\langle\nabla f\left(y^{k}\right), \alpha_{k}\left(v^{k+1}-v^{k}\right)\right\rangle .
$$

De (3.45) segue que $\alpha_{k}\left(v^{k+1}-v^{k}\right)=\hat{x}-y^{k}$. Logo, da hipótese que $D_{h}(\cdot, \cdot)$ é fortemente convexa com parâmetro $\sigma$, podemos escrever a seguinte expressão:

$$
D_{h}\left(v^{k+1}, v^{k}\right) \geq \frac{\sigma}{2}\left\|v^{k+1}-v^{k}\right\|^{2}=\frac{\sigma}{2 \alpha_{k}^{2}}\left\|\hat{x}-y^{k}\right\|^{2} .
$$

Substituindo em (3.48) chegamos em

$$
\phi_{k+1}^{*} \geq f\left(y^{k}\right)+\frac{\sigma \gamma_{k+1}}{2 \alpha_{k}^{2}}\left\|\hat{x}-y^{k}\right\|^{2}+\left\langle\nabla f\left(y^{k}\right), \hat{x}-y^{k}\right\rangle .
$$

Pela proposição 3.20 sabemos que

$$
f\left(y^{k}\right)+\left\langle\nabla f\left(y^{k}\right), \hat{x}-y^{k}\right\rangle+\frac{L^{a}}{2}\left\|\hat{x}-y^{k}\right\|^{2} \geq f(\hat{x}) .
$$

Substituindo em (3.49)

$$
\phi_{k+1}^{*} \geq f(\hat{x})+\left(\frac{\sigma \gamma_{k+1}}{2 \alpha_{k}^{2}}-\frac{L^{a}}{2}\right)\left\|\hat{x}-y^{k}\right\|^{2} .
$$


Como $\alpha_{k}$ satisfaz a equação $L^{a} \alpha_{k}^{2}=\sigma\left(1-\alpha_{k}\right) \gamma_{k}$ temos que o segundo termo do lado direito da desigualdade é nulo. Portanto, como $x^{k+1}$ é tal que $f\left(x^{k+1}\right) \leq f(\hat{x})$, concluímos que

$$
\phi_{k+1}^{*} \geq f\left(x^{k+1}\right)
$$

A demonstração que $\alpha_{k} \in(0,1)$ é idêntica a demonstração feita no teorema 2.8 .

Temos deste teorema e do lema 3.19 que as sequências definidas pelo nosso método verificam a desigualdade (3.3). Nos próximos resultados mostraremos que o nosso método é ótimo. Abaixo, apresentamos uma descrição completa do novo método:

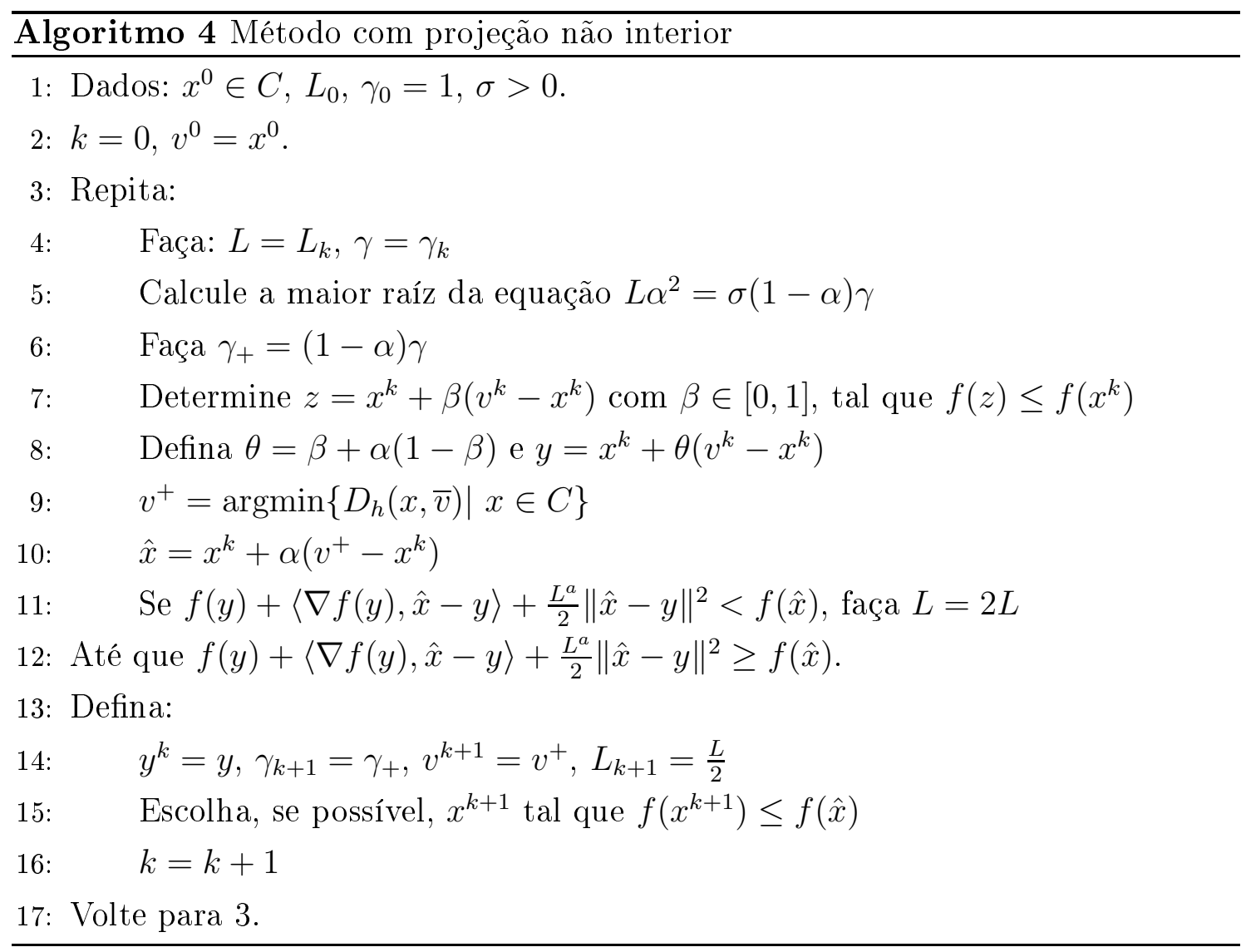

Pela proposição 3.20 , se $L^{a}$ não foi aceito pelo algoritmo, sabemos que $L^{a}<$ $L$. Como o próximo valor testado pelo algoritmo é sempre o dobro do valor anterior podemos concluir que $L^{a}$ nunca é maior que duas vezes o valor verdadeiro de $L$ e assim podemos dizer que $L^{a} \leq 2 L$. 
Observe que em cada iteração do laço interno do algoritmo o gradiente é avaliado apenas uma vez e são feitas duas avaliações de função. Logo, usando o fato que $L^{a} \leq 2 L$, como o nosso mecanismo para adaptar a constante de Lipschitz é similar ao de Nesterov, obtemos que o número total de avaliações de gradiente $\left(N G_{k}\right)$ depois de $k$ iterações é

$$
N G_{k} \leq 2(k+1)+\log _{2} \frac{L}{L_{0}}
$$

E o número total de avaliações de função $\left(N F_{k}\right)$ depois de $k$ iterações é

$$
N F_{k} \leq 4(k+1)+\log _{2} \frac{L}{L_{0}}
$$

Isto garante que o laço interno será feito em número finito e constante de vezes.

Os próximos resultados, que garantem a otimalidade do nosso método, são equivalentes ao lema 3.10 e ao teorema 3.11 exceto pelo fato que estamos usando um valor aproximado para a constante de Lipschitz com a garantia que este valor nunca é maior que duas vezes o valor verdadeiro de $L$.

Lema 3.22. Sejam $\gamma_{k} \geq 0$ e $\alpha_{k} \in(0,1)$ definidos por $L^{a} \alpha_{k}^{2}=\sigma\left(1-\alpha_{k}\right) \gamma_{k} e$ $\gamma_{k+1}=\left(1-\alpha_{k}\right) \gamma_{k}$. Como $\lambda_{k}:=\prod_{i=0}^{k-1}\left(1-\alpha_{i}\right)$, então

$$
\lambda_{k} \leq \frac{8 L}{\left(2 \sqrt{2 L}+k \sqrt{\sigma \gamma_{0}}\right)^{2}}
$$

Demonstração:

Note que $\gamma_{k}=\gamma_{0} \lambda_{k}$, para todo $k \geq 0$. Desta forma, $L^{a} \alpha_{k}^{2}=\sigma \gamma_{k+1}=\sigma \gamma_{0} \lambda_{k+1}$, e do fato que $L^{a} \leq 2 L$, temos quer

$$
\begin{aligned}
& 2 L \alpha_{k}^{2} \geq \sigma \gamma_{0} \lambda_{k+1} \\
& \frac{\alpha_{k}}{\sqrt{\lambda_{k+1}}} \geq \sqrt{\frac{\sigma \gamma_{0}}{2 L}}
\end{aligned}
$$

Denotamos $a_{k}=\frac{1}{\sqrt{\lambda_{k}}}$. Análogo ao lema 2.9, podemos provar que

$$
a_{k+1}-a_{k} \geq \frac{\alpha_{k}}{2 \sqrt{\lambda_{k+1}}} .
$$


Logo,

$$
a_{k+1}-a_{k} \geq \frac{1}{2} \sqrt{\frac{\sigma \gamma_{0}}{2 L}}
$$

Temos então que $a_{k+1}-a_{k} \geq \frac{1}{2} \sqrt{\frac{\sigma \gamma_{0}}{2 L}}$. Logo, $a_{k}-a_{0} \geq \frac{k}{2} \sqrt{\frac{\sigma \gamma_{0}}{2 L}}$. Ou seja

$$
\begin{aligned}
a_{k} & \geq 1+\frac{k}{2} \sqrt{\frac{\sigma \gamma_{0}}{2 L}} \\
\frac{1}{\sqrt{\lambda_{k}}} & \geq \frac{2 \sqrt{2 L}+k \sqrt{\sigma \gamma_{0}}}{2 \sqrt{2 L}} \\
\lambda_{k} & \leq \frac{8 L}{\left(2 \sqrt{2 L}+k \sqrt{\sigma \gamma_{0}}\right)^{2}} .
\end{aligned}
$$

De onde segue (3.50).

Teorema 3.23. Sejam $\gamma_{0}=1$ e $\left\{x^{k}\right\},\left\{y^{k}\right\}$ e $\left\{\alpha_{k}\right\}$ as sequências geradas pelo algoritmo 4 e seja $x^{*}$ a solução ótima do problema 3.1. Então para qualquer $k \geq 0$ temos

$$
f\left(x^{k}\right)-f\left(x^{*}\right) \leq \frac{8 L}{\sigma \gamma_{0} k^{2}} C\left(x^{*}, x^{0}\right)=O\left(\frac{1}{k^{2}}\right)
$$

em que $C\left(x^{*}, x^{0}\right)=D_{h}\left(x^{*}, x^{0}\right)+f\left(x^{0}\right)-f\left(x^{*}\right)$.

Demonstração:

Como $\gamma_{0}=1$, do lema 3.19 e do teorema 3.21 temos que as condições (3.4) e (3.5) são satisfeitas e desta forma a sequência $\left\{x^{k}\right\}$ satisfaz a seguinte desigualdade,

$$
f\left(x^{k}\right)-f\left(x^{*}\right) \leq \lambda_{k}\left(\phi_{0}\left(x^{*}\right)-f\left(x^{0}\right)\right) .
$$

Como $\phi_{0}\left(x^{*}\right)=f\left(x^{0}\right)+\gamma_{0} D_{h}\left(x^{*}, x^{0}\right)$, podemos escrever a seguinte expressão

$$
f\left(x^{k}\right)-f\left(x^{*}\right) \leq \lambda_{k} C\left(x^{*}, x^{0}\right) .
$$

Pelo lema 3.22 obtemos

$$
\lambda_{k} \leq \frac{8 L}{(2 \sqrt{2 L}+k \sqrt{\sigma})^{2}} \leq \frac{8 L}{\sigma \gamma_{0} k^{2}}
$$


De onde ocorre (3.51).

Portanto, o novo método encontra aproximadamente uma solução ótima $x^{*}$, com precisão $\epsilon$, em um número proporcional a $\frac{1}{\sqrt{\epsilon}}$ iterações, garantindo, assim, a sua otimalidade.

No próximo capítulo exibimos os testes numéricos realizados para comprovar a eficiência do novo método. 


\section{Capítulo 4}

\section{Testes Numéricos}

No capítulo anterior propomos um novo método ótimo baseado nos algoritmos de Nesterov e Auslender e Teboulle para resolver o problema de minimização de uma função convexa restrita a um conjunto convexo simples. Entretanto, como vimos no algoritmo 4, o nosso método nos dá liberdade de fazermos algumas escolhas. Na seção 4.2, mostramos algumas possibilidades para estas escolhas buscando sempre avaliar a sua eficiência numérica. Ao final mostramos uma comparação das melhores versões de nossa estratégia com o método de Nesterov. As implementações foram feitas na linguagem do Matlab.

\subsection{Detalhes de implementação}

\subsubsection{Problemas testados}

Os testes numéricos foram feitos em três classes de problemas convexos com restrições de caixa, ou seja,

$$
C=\left\{x \in \mathbb{R}^{n} \mid l \leq x \leq u\right\}
$$

$\operatorname{com} l, u \in \mathbb{R}^{n}$ e $l \leq u$.

- No artigo Projected Barzilai-Borwein methods for large-scale box-constrained quadratic programming, Dai e Flecther descrevem uma forma de gerar problemas convexos aleatoriamente [5]. Em nossos testes geramos 162 problemas aleatórios, com dimensão $n=100$ e valor para a constante de Lipschitz $L$ variando entre 1000 e 100000 . 
- No artigo On the solution of large quadratic programming problems with bound constraints [12] Moré e Toraldo apresentam os seguintes problemas convexos:

(a) The obstacle,

(b) The elastic-plastic torsion,

(c) The journal bearing,

Na biblioteca de problemas CUTEr estão disponíveis 21 variações destes problemas, os problemas The obstacle tem dimensão 5476 e os problemas The elastic-plastic torsion e the journal bearing tem dimensão 10000.

- Dentro da biblioteca CUTEr existe um subcoleção de problemas chamada Maros e Meszaros. Esta subcoleção possui 140 problemas de otimização quadrática convexa cujo conjunto de restrições é formado por restrições de caixa e restrições lineares. Inicialmente, resolvemos estes problemas (por um método diferente dos métodos trabalhados nesta tese) desprezando as restrições lineares e analisamos onde se encontra a solução ótima. Selecionamos os problemas cuja solução ótima não é um ponto interior e nem um vértice do conjunto viável, totalizando 16 problemas. Os demais problemas foram excluídos pois consideramos não serem os casos interessantes para validar nossa teoria, uma vez que o caso em que o minimizador irrestrito pertence ao conjunto corresponde a uma problema de otimização irrestrita e quando o minimizador esta em um vértice o método termina em tempo finito. A dimensão destes problemas varia entre 2 e 10197.

Pelo fato de serem problemas reais daremos preferência ao segundo grupo de problemas caso haja algum tipo de contradição nos resultados.

\subsubsection{Critério de Comparações}

Para comparar diferentes métodos fizemos os gráficos de perfil de desempenho [6]. Poderíamos usar como critério de comparação o número de iterações. Porém, consideramos que este não é um critério justo pois o trabalho por iteração pode variar de método para método. Por exemplo, o método de Nesterov descrito no início do capítulo anterior nunca avalia o valor de função objetivo, enquanto que nossos métodos podem fazer muitas avaliações de função pois permitem buscas nas escolhas dos pontos $y^{k}$ e $x^{k+1}$. 
Sendo assim usamos como critério de comparação uma média ponderada entre o número de avaliações de função e o número de avaliações de gradiente:

$$
\text { Média }=\operatorname{aval} G+\frac{1}{3} \max \{0, \text { aval } F-\operatorname{aval} G\} .
$$

Neste critério estamos considerando o número de avaliações de gradiente mais um terço do número de vezes que o método avalia a função sem avaliar o gradiente. A escolha deste critério é justificado pelo trabalho de Griewank [9]. Este artigo nos garante que usando diferenciação automática a avaliação do gradiente de uma função em um ponto é no máximo cinco vezes mais cara que avaliar o valor da função. Como 5 é um limitante superior, usamos um fator de ponderação menor para não penalizar as avaliações de função pelo pior caso, dividindo a diferença por três.

\subsubsection{Critério de Parada}

Como critério de parada usamos que a norma infinito do gradiente projetado a partir do ponto $x^{k}$ seja menor que uma precisão $\epsilon$, ou seja,

$$
\left\|x^{k}-\operatorname{Proj}_{C}\left(x^{k}-\nabla f\left(x^{k}\right)\right)\right\|_{\infty} \leq \epsilon .
$$

Porém, muitos métodos, entre eles o método de Auslender e Teboulle, avaliam em todas as iterações apenas o gradiente da função objetivo no ponto $y^{k}$. Sendo assim, para evitar o cômputo de um gradiente extra, nestes esquemas adotamos como critério de parada que a norma infinito do gradiente projetado a partir do ponto $y^{k}$ seja menor que uma precisão $\epsilon$, ou seja,

$$
\left\|y^{k}-\operatorname{Proj}_{C}\left(y^{k}-\nabla f\left(y^{k}\right)\right)\right\|_{\infty} \leq \epsilon
$$

Usamos ainda um limitante para o número de iterações, kmax. Na tabela abaixo exibimos o valor de $\epsilon$ e de $k$ max que foi usado em cada classe de problemas:

\begin{tabular}{c|cc}
\hline & $\epsilon$ & $\operatorname{kmax}$ \\
\hline Aleatórios & $10^{-4}$ & 100000 \\
Cuter & $10^{-5}$ & 100000 \\
Maros e Meszaros & $10^{-5}$ & 300000 \\
\hline
\end{tabular}


Usamos um valor de $\epsilon$ diferente nos problemas aleatórios pois para valores altos de $L$ o número de iterações e consequentemente o tempo computacional aumentam significativamente.

\subsection{Comparações}

\subsubsection{Critério de aceitação para $L_{a}$}

No algoritmo 4, uma passagem deixada em aberto é o critério de aceitação para $L_{a}$. Da proposição 3.20 temos que se $L_{a} \geq L$, então

$$
f\left(y^{k}\right)+\left\langle\nabla f\left(y^{k}\right), x^{k+1}-y^{k}\right\rangle+\frac{L^{a}}{2}\left\|x^{k+1}-y^{k}\right\|^{2} \geq f\left(x^{k+1}\right) .
$$

E pelo teorema 3.21, com este resultado podemos garantir que $\phi_{k+1}^{*} \geq f\left(x^{k+1}\right)$.

Desta forma podemos concluir que se a desigualdade (4.2) não é satisfeita, significa que $L_{a}<L$. Então $L_{a}$ deve ser aumentado até verificar (4.2) e em consequência garantir que $\phi_{k+1}^{*} \geq f\left(x^{k+1}\right)$. Sendo assim, a desigualdade (4.2) pode ser usada como critério para obter $L_{a}$ de forma adaptativa sem comprometer a otimalidade do nosso método.

Entretanto, a desigualdade (4.2) é usada para garantir que $\phi_{k+1}^{*} \geq f\left(x^{k+1}\right)$. Porém, sabemos que se em uma determinada iteração do algoritmo 4, após as escolhas de $\alpha_{k}, x^{k}, y^{k}$ e $v^{k}$, ocorrer que $\phi_{k+1}^{*}<f\left(x^{k+1}\right)$ significa que $L_{a}<L$. Sendo assim, podemos também usar como critério de aceitação para $L_{a}$ que a desigualdade $\phi_{k+1}^{*} \geq f\left(x^{k+1}\right)$ seja satisfeita.

Retomando o que foi dito da seção 3.3.2, se o valor estimado de $L$ não for suficiente para passar no teste, dobramos o valor estimado e repetimos isto até encontrar um valor (que estamos chamando de $L_{a}$ ) que passe no teste. Na iteração seguinte o primeiro valor testado será a metade do valor de $L_{a}$ anterior.

\subsubsection{Escolha da distância de Bregman}

Como vimos na seção 3.3, o uso de distâncias de Bregman com zonas maiores que o conjunto viável permite projetar sobre a fronteira do conjunto viável e, em particular permite o uso da distância euclidiana ao quadrado como distância de Bregman. 
Em nossos primeiros testes numéricos, fizemos uma comparação entre o método de Auslender e Teboulle, usando uma variação da distância de KullbackLeibler para caixas, denotado por PassoAT-KL,

$$
D_{h}(x, y)=\sum_{i=1}^{n}\left(\left(x_{i}-a_{i}\right) \ln \frac{x_{i}-a_{i}}{y_{i}-a_{i}}+\left(b_{i}-x_{i}\right) \ln \frac{b_{i}-x_{i}}{b_{i}-y_{i}}\right)
$$

e uma versão deste método trocando esta distância pela norma euclidiana ao quadrado, que chamamos PassoAT-E,

$$
D_{h}(x, y)=\frac{1}{2}\|x-y\|^{2}
$$

A distância (4.3) foi escolhida por obedecer todas as hipóteses que o método de Auslender e Teboulle exige [16]. Porém, esta distância exige que alguns cuidados sejam tomados durante sua implementação. Isto porque, embora a teoria garanta que o ponto $v^{k}$ determinado usando esta distância está no interior do conjunto viável, computacionalmente o resultado pode ser um ponto da fronteira deste. Desta forma, a operação para determinar $v^{k+1}$ na próxima iteração, não estaria definida. Então, se do cálculo de $v^{k}$ resultar um ponto que está na fronteira do conjunto viável, assumimos que $v^{k}$ é o limitante na fronteira mais $10^{-8}$.

Abaixo exibimos uma comparação entre estes dois métodos para resolver o grupo de problemas aleatórios. Os outros dois grupos foram excluídos pois observamos que em muitos deles a restrição de caixa é ilimitada em um dos extremos. Neste caso a distância de Bregman é

$$
D_{h}(x, y)=\sum_{i=1}^{n}\left(\left(x_{i}-a_{i}\right) \ln \frac{x_{i}-a_{i}}{y_{i}-a_{i}}\right)+\frac{1}{2}\|x-y\|^{2},
$$

e a projeção interior associada a esta função envolve a função $W$ de Lambert cuja implementação em Matlab é muito lenta, inviabilizando os testes. 


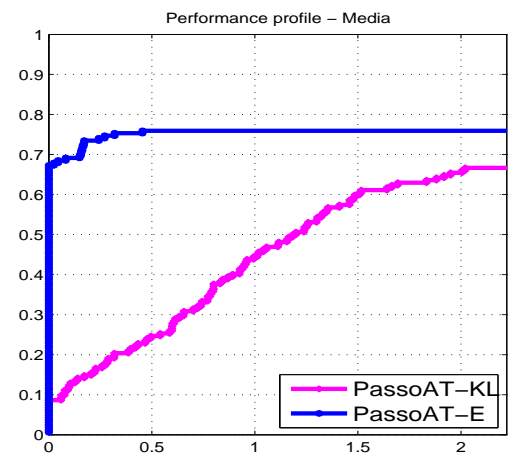

(a) Aleatórios

Figura 4.1: (PassoAT $-\mathrm{KL}) \times($ PassoAT $-\mathrm{E})$

Aqui fazemos um breve comentário sobre como interpretar um gráfico de perfil de desempenho. A esquerda do gráfico é medida a eficiência dos métodos (porcentagem de vezes que um método foi mais eficiente), enquanto que os valores do extremo direito nos dizem sobre a robustez dos métodos (número de problemas que foram resolvidos por cada um dos métodos). De forma geral, se $(X, Y)$ pertence ao gráfico associado ao método $M$, isso quer dizer que $M$ não leva mais do que $X$ vezes o tempo do melhor método para resolver $(Y \times 100) \%$ de todos problemas. Em todos os gráficos usamos escala logarítmica, considerando 2 como base.

Sendo assim, é possível observar que o esquema PassoAT-E é o mais eficiente em quase todos os problemas que foram resolvidos dentro do número de iterações adotado.

Refletindo um pouco sobre estes métodos, constatamos que a medida que o algoritmo evolui os passos entre as iterações ficam cada vez menores fazendo com que seja preciso muitas iterações para satisfazer o critério de parada. Vamos ilustrar que até mesmo em situações muito favoráveis o método de Auslender e Teboulle (independente da distância escolhida) sempre se comporta como no pior caso, considerado em sua taxa de convergência. Para isso, relembrando o capítulo anterior, temos que Auslender e Teboulle escolhem $x^{k+1}$ como:

$$
x^{k+1}=x^{k}+\alpha_{k}\left(v^{k}-x^{k}\right) \text {. }
$$

Vamos analisar o que acontece em um caso que nos pareceu particularmente favorável: admitindo que $x^{*}$ é uma solução, vamos supor que em todas iterações $v^{k}=x^{*}$. Temos, então, que na primeira iteração: 


$$
\begin{aligned}
x^{1} & =x^{0}+\alpha_{0}\left(x^{*}-x^{0}\right) \\
& =\alpha_{0} x^{*}+\left(1-\alpha_{0}\right) x^{0}
\end{aligned}
$$

Logo, na segunda iteração obtemos:

$$
\begin{aligned}
x^{2} & =x^{1}+\alpha_{1}\left(x^{*}-x^{1}\right) \\
& =\alpha_{1} x^{*}+\left(1-\alpha_{1}\right) x^{1} \\
& =\alpha_{1} x^{*}+\left(1-\alpha_{1}\right)\left(\alpha_{0} x^{*}+\left(1-\alpha_{0}\right) x^{0}\right) \\
& =\left(\alpha_{1}+\left(1-\alpha_{1}\right) \alpha_{0}\right) x^{*}+\left(1-\alpha_{1}\right)\left(1-\alpha_{0}\right) x^{0}
\end{aligned}
$$

Graficamente, isto corresponde a seguinte situação:

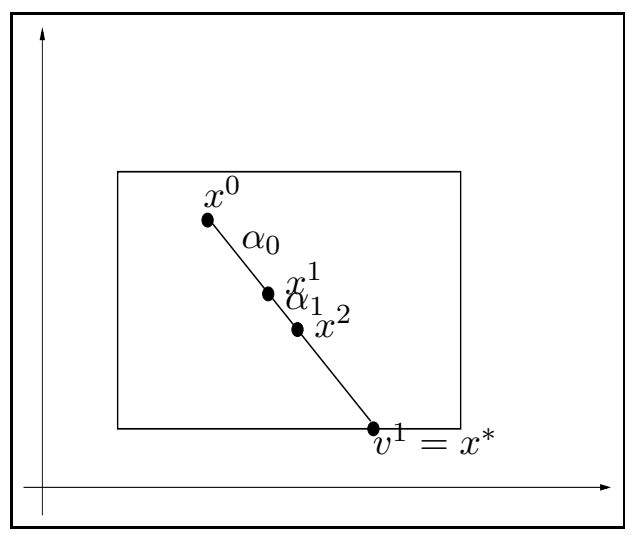

Figura 4.2: Comportamento do método Auslender e Teboulle

Como, por definição, $\lambda_{k}=\prod_{i=0}^{k-1}\left(1-\alpha_{i}\right)$ e $x^{2}$ é uma combinação convexa entre $x^{0} \mathrm{e}$ $x^{*}$, temos que:

$$
x^{2}=\left(1-\lambda_{2}\right) x^{*}+\lambda_{2} x^{0} .
$$

Continuando este processo, chegamos à seguinte expressão para $x^{k}$, quando $v^{k}=x^{*}$ em todas iterações,

$$
x^{k}=\left(1-\lambda_{k}\right) x^{*}+\lambda_{k} x^{0} .
$$

Como $\lambda_{k}=O\left(\frac{1}{k^{2}}\right)$, temos que $\left\|x^{k}-x^{*}\right\|=O\left(\frac{1}{k^{2}}\right)$, ou seja, $x^{k}$ se aproxima de $x^{*}$ exatamente na velocidade estimada para o pior caso. 
Portanto, é fundamental buscar alternativas para evitar passos curtos buscando decrescer mais rápido o valor da função objetivo. Na próxima seção mostramos como determinar o ponto $x^{k+1}$ de modo a aumentar a eficiência dos métodos.

\subsubsection{Como determinar novos pontos}

Como foi dito no início desta seção precisamos fazer escolhas para os pontos de forma a melhorar a eficiência do algoritmo. Nesta seção mostramos como determinar o ponto $x^{k+1}$ melhorando a eficiência do algoritmo 4 .

Como vimos na seção 3.3.1, podemos escolher $x^{k+1}$ tal que $f\left(x^{k+1}\right) \leq f(\hat{x})$, onde $\hat{x}=x^{k}+\left(1-\alpha_{k}\right)\left(v^{k}-x^{k}\right)$ é o ponto sugerido pelo método de Auslender e Teboule. Vamos denotar provisoriamente por $\tilde{x}$ o ponto escolhido como tentativa de melhorar $\hat{x}$, ou seja, $f(\tilde{x}) \leq f(\hat{x})$. Como já temos disponível o gradiente da função objetivo avaliado no ponto $y^{k}$, a primeira ideia que tivemos para $\tilde{x}$ foi a seguinte projeção a partir do ponto $y^{k}$ :

$$
\tilde{x}=\operatorname{Proj}_{C}\left(y^{k}-\xi \nabla f\left(y^{k}\right)\right)
$$

com $\xi=\frac{1}{L_{a}}$. Depois de obter este ponto comparamos os valores $f(\tilde{x})$ e $f(\hat{x})$. Se $f(\tilde{x}) \leq f(\hat{x})$, então escolhemos $x^{k+1}=\tilde{x}$, caso contrário $x^{k+1}=\hat{x}$.

No gráfico abaixo, comparamos uma versão do método de Auslender e Teboulle usando a distância euclidiana e uma versão do algoritmo 4 usando como candidato a $x^{k+1}$ o ponto descrito acima (esta versão é representada pela sigla xLy). 


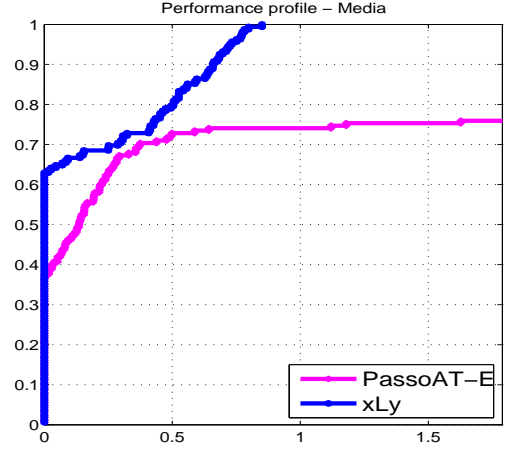

(a) Aleatórios

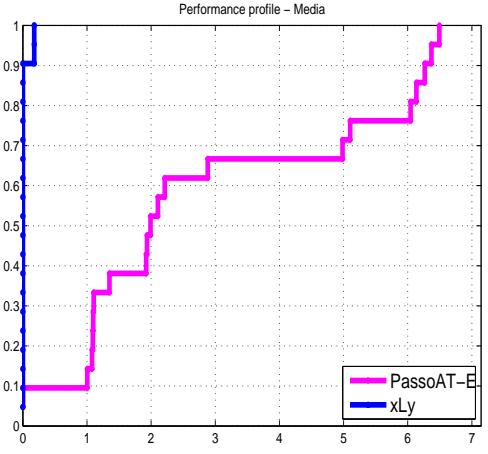

(b) Moré e Toraldo

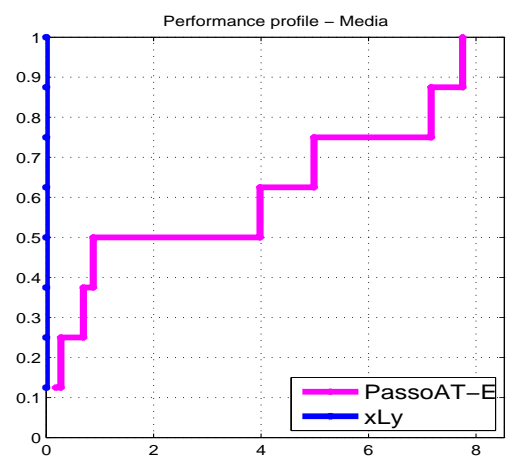

(c) Maros e Mezsaros

Figura 4.3: $($ PassoAT $-\mathrm{E}) \times(\mathrm{xLy})$

Claramente observamos que o esquema xLy é mais eficiente que o esquema PassoAT-E. Por exemplo, em muitos dos problemas Moré e Toraldo o procedimento PassoAT-E trabalhou 16 vezes mais que o procedimento xLy.

Outra possibilidade é tentar projetar a partir do ponto $x^{k}$, ou seja,

$$
\tilde{x}=\operatorname{Proj}_{C}\left(x^{k}-\frac{1}{L_{a}} \nabla f\left(x^{k}\right)\right) .
$$

Neste caso, estaremos fazendo uma avaliação de gradiente a mais por iteração. Abaixo apresentamos uma comparação entre o método que faz projeções a partir de $y^{k}(\mathrm{xLy})$ e o método que faz projeções a partir de $x^{k}(\mathrm{xLx})$ : 


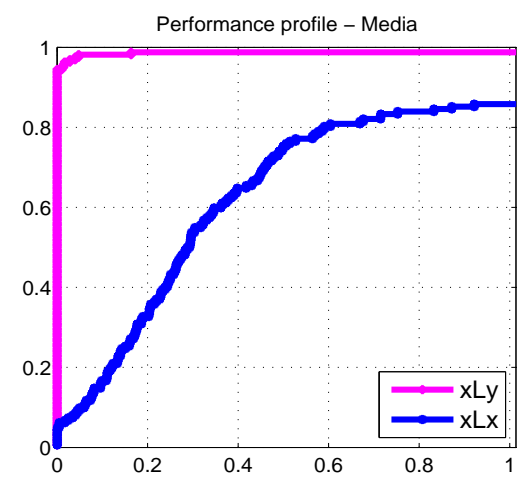

(a) Aleatórios

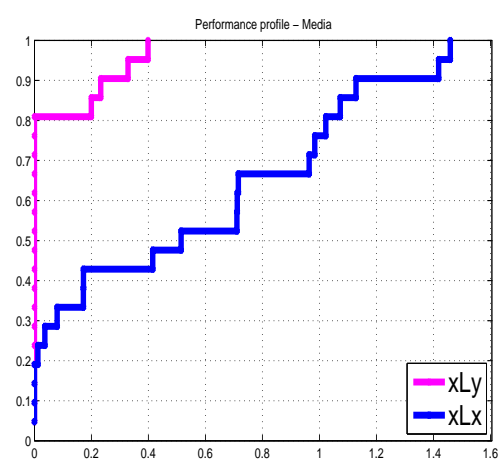

(b) Moré e Toraldo

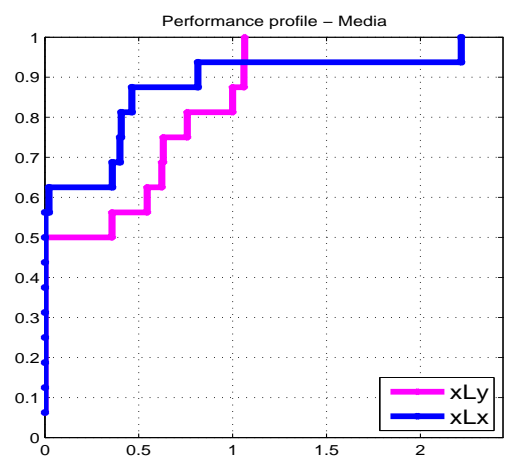

(c) Maros e Mezsaros

Figura 4.4: $(x L y) \times(x L x)$

Analisando estes gráficos vemos uma superioridade do método xLy nas duas primeiras classes de problema. Embora o método xLx tenha sido mais eficiente nos problemas Maros e Mezsaros, o ganho não foi muito significativo. Sendo assim, os testes sugerem que o método xLy é mais eficiente. Logo é razóavel afirmar que usando o passo $\frac{1}{L_{a}}$, é mais eficiente fazer projeções em relação ao ponto $y^{k}$. Desta forma, no restante desta seção, sempre que comparamos métodos usando passos $\frac{1}{L_{a}}$ a projeção está sendo feita a partir do ponto $y^{k}$.

Agora, retomando a discussão da seção 4.2.1, uma outra variação possível do algoritmo 4 é usar como critério de aceitação para $L_{a}$ que a desigualdade $\phi_{k+1}^{*} \geq$ $f\left(x^{k+1}\right)$ seja satisfeita. Sempre que usamos este critério o identificamos pelo número 2. Abaixo exibimos uma comparação envolvendo o método que chamamos de xLy e o método que usa o critério $\phi_{k+1}^{*} \geq f\left(x^{k+1}\right)$, designado por xLy-2, ambos com passo $x^{k+1}$ obtido através de projeção a partir de $y^{k}$. 


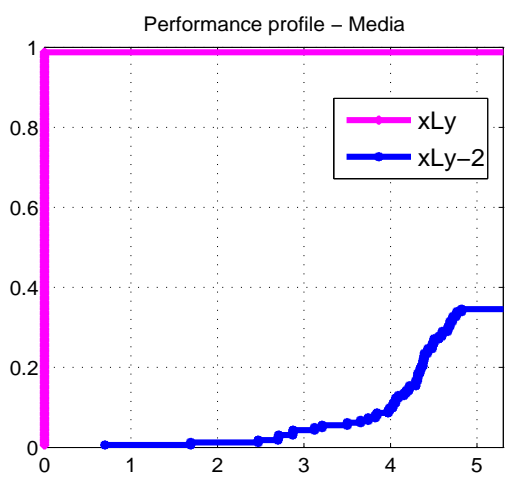

(a) Aleatórios

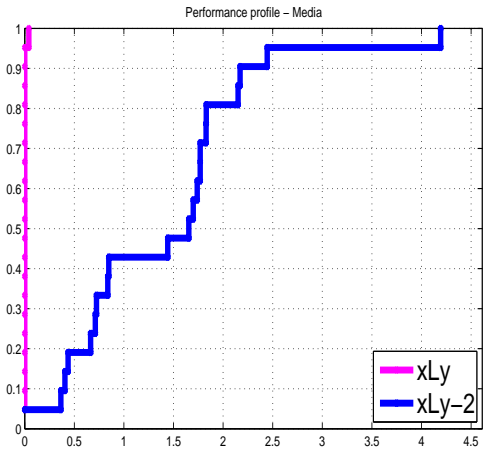

(b) Moré e Toraldo

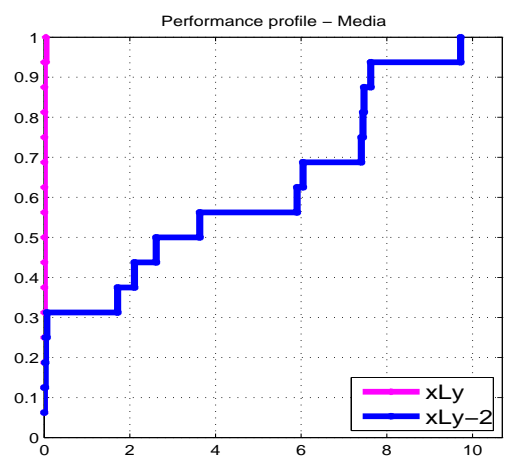

(c) Maros e Mezsaros

Figura 4.5: $(x L y) \times(x L y-2)$

É possível constatar que o esquema xLy é mais eficiente em todas classes de problemas e em grande parte dos problemas esta superioridade passa de uma ordem de grandeza. Sendo assim, constatamos que escolhendo o ponto

$$
\tilde{x}=\operatorname{Proj}_{C}\left(y^{k}-\frac{1}{L_{a}} \nabla f\left(y^{k}\right)\right),
$$

o critério dado por (4.2) torna o método mais eficiente.

Observe entretanto, que na parte (a) da figura acima nos deparamos com o problema de falta de robustez, dentro do número de iterações estipuladas, do método xLy-2. Refizemos esta comparação multiplicando o número máximo de iterações por 10 e o número de problemas resolvidos aumentou significativamente, o que nos leva a concluir que aumentando ainda mais o número de iterações seria possível completar a resolução de todos os problemas. Entretanto para resolver este conjunto de problemas com tal número de iterações foi preciso quase 3 dias, inviabilizando os testes com mais iterações. 
Por fim, observando as linhas 5 e 6 do algoritmo 4, notamos a possibilidade de determinar $y^{k}$ usando um passo $\theta \geq \alpha_{A T}$. Para isto é preciso determinar um ponto $z$ entre $x^{k}$ e $v^{k}$ de modo que $f(z) \leq f\left(x^{k}\right)$. Seguindo a seção 3.3.1, nessa etapa dos nossos testes, optamos em fazer uma busca ao longo da direção $\left(v^{k}-x^{k}\right)$ começando com tamanho de passo $\beta=1$. Se com este passo não é possível satisfazer $f(z) \leq f\left(x^{k}\right)$, reduzimos $\beta$ pela metade. Para evitar um custo computacional alto, são feitas apenas três tentativas, caso esta busca não encontre $z$ com valor de função menor que o valor de função de $x^{k}$ o método continua tomando $\beta=0$. Este método é representado pela sigla xLy-yb.

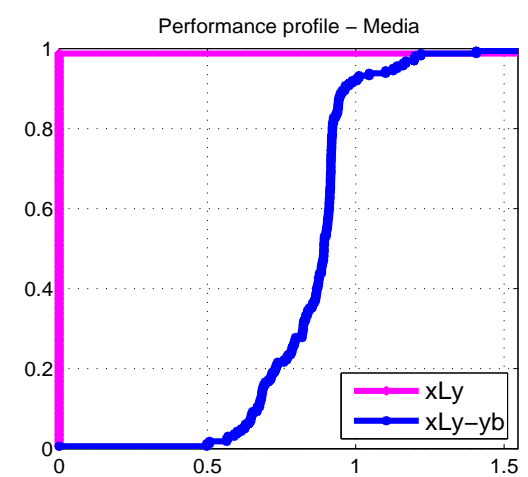

(a) Aleatórios

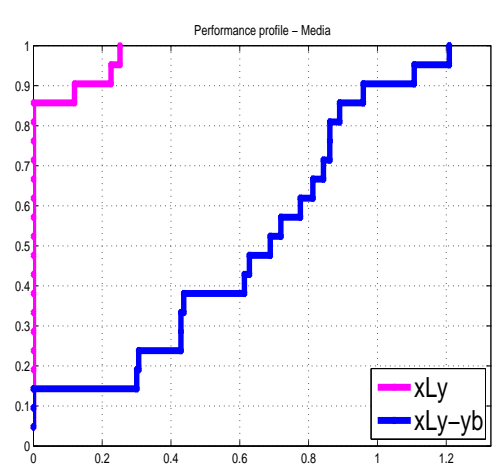

(b) Moré e Toraldo

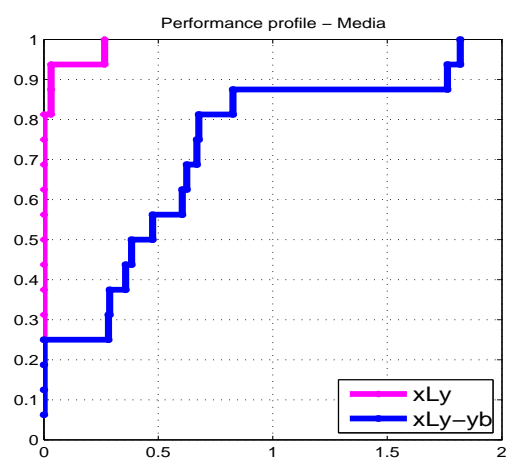

(c) Maros e Mezsaros

Figura 4.6: $(x L y) \times(x L y-y b)$

Estes testes nos mostram que esta busca para determinar um passo $\theta \geq \alpha_{A T}$ não melhora a eficiência do método em relação à escolha do ponto $y^{k}$ determinado por Auslender e Teboulle. Sendo assim, podemos decidir, que a melhor versão quando usamos o tamanho do passo $\xi=\frac{1}{L_{a}}$ é a versão que faz a projeção a partir de $y^{k}$ e usa a desigualdade (4.2) como critério de aceitação para $L_{a}$. No gráfico abaixo, comparamos esta versão com o método ótimo de Nesterov (Nest): 


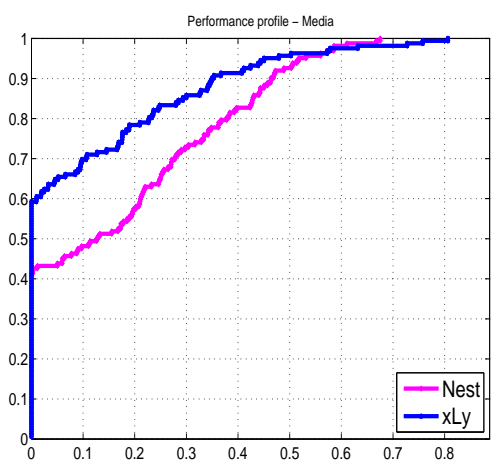

(a) Aleatórios

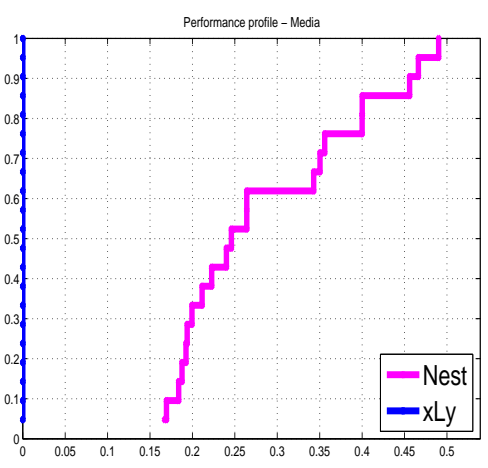

(b) Moré e Toraldo

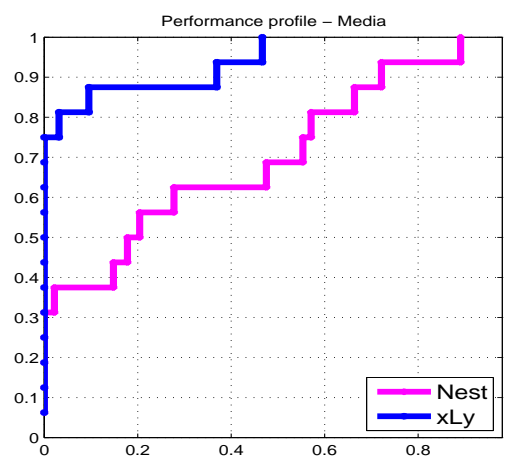

(c) Maros e Mezsaros

Figura 4.7: (Nest) $\times(\mathrm{xLy})$

Podemos verificar que ambos métodos conseguem resolver todos os problemas e claramente esta versão do algoritmo 4 foi mais eficiente nas três classes de problemas, porém a diferença não é muito grande.

\subsubsection{Introduzindo o passo SPG}

Todos os testes acima foram feitos considerando sempre um tamanho do passo $\xi=\frac{1}{L_{a}}$. Entretanto podemos testar outras escolhas para o tamanho de passo $\xi$. Nesta seção, nosso objetivo é desenvolver um método que tenta aproveitar, quando possível, o passo espectral definido no trabalho de Birgin, Martínez e Raydan [4], porém com a garantia de otimalidade. Para isto, se a projeção está sendo feita a partir de $y^{k}$, o passo $\xi$ pode ser definido pelo seguinte quociente:

$$
\xi_{s p g}:=\frac{\left\langle y^{k}-y^{k-1}, y^{k}-y^{k-1}\right\rangle}{\left\langle y^{k}-y^{k-1}, \nabla f\left(y^{k}\right)-\nabla f\left(y^{k-1}\right)\right\rangle} .
$$

Neste caso, é possível testar uma versão com busca na direção oposta ao gradiente 
da função objetivo. Para esta busca é conveniente definir

$$
\begin{gathered}
\tilde{d}_{k}=\operatorname{Proj}_{C}\left(y^{k}-\xi_{s p g} \nabla f\left(y^{k}\right)\right)-y^{k}, e \\
\tilde{x}=y^{k}+\rho \tilde{d}_{k} .
\end{gathered}
$$

Assim como em [4], usamos uma busca baseada em interpolação quadrática, com salvaguardas e que procura um passo $\rho$ que garante que $f(\tilde{x}) \leq \max \left\{f(\hat{x}), f\left(y^{k}\right)\right\}$. Usamos o máximo pois, embora a busca garanta um ponto com valor de função menor que o valor em $y^{k}$, é suficiente parar quando encontramos um ponto cujo valor de função seja menor que $f(\hat{x})$. Para limitar o número de passos, nesta busca impedimos que $\rho$ fique menor que $10^{-3}$. Se a busca parou porque $\rho$ esta menor que $10^{-3}$, então tomamos $x^{k+1}=\hat{x}$, caso contrário $x^{k+1}=\tilde{x}$. Sendo assim, podemos definir mais quatro variações do nosso método (considerando a projeção a partir de $\left.y^{k}\right)$ :

- xSPGy: $\tilde{x}$ com passo SPG definido por (4.7), sem busca, e $L_{a}$ definido pelo critério (4.2);

- xSPGy-2: $\tilde{x}$ com passo SPG definido por (4.7), sem busca, e $L_{a}$ definido pelo critério $\phi_{k+1}^{*} \geq f\left(x^{k+1}\right)$;

- xSPGyb: $\tilde{x}$ com passo SPG definido por (4.7), com busca, e $L_{a}$ definido pelo critério (4.2);

- xSPGyb-2: $\tilde{x}$ com passo SPG definido por (4.7), com busca, e $L_{a}$ definido pelo critério $\phi_{k+1}^{*} \geq f\left(x^{k+1}\right)$. 


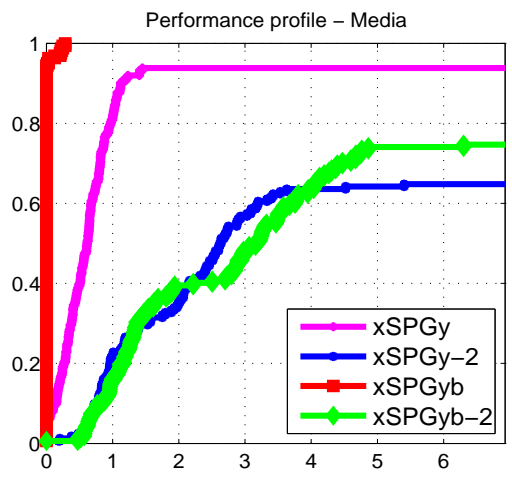

(a) Aleatórios

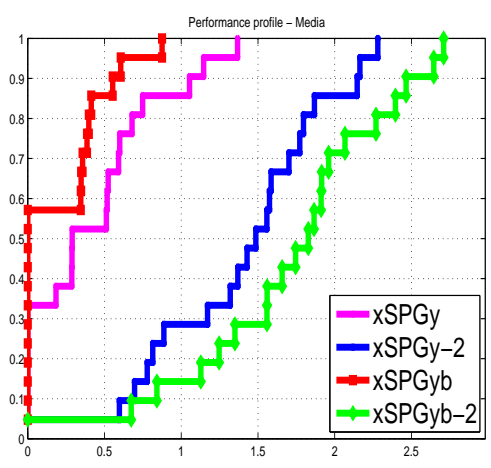

(b) Moré e Toraldo

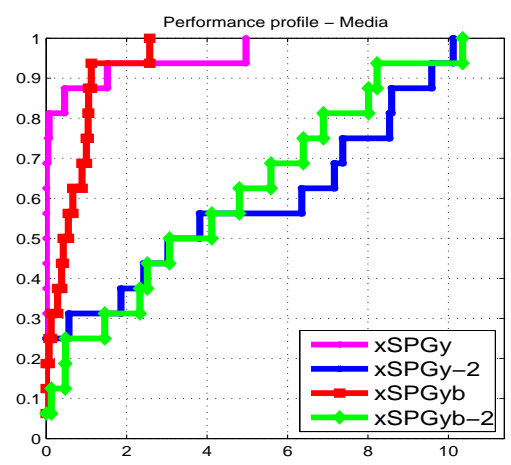

(c) Maros e Mezsaros

Figura 4.8: Métodos com projeção em relação a $y^{k}$ e passo $\xi_{s p g}$

Observamos que os esquemas mais eficientes foram, mais uma vez, aqueles que usaram a desigualdade (4.2) como critério de aceitação para $L_{a}$. Para visualizar melhor o desempenho destes métodos refizemos o gráfico apenas com xSPGy e xSPGyb: 


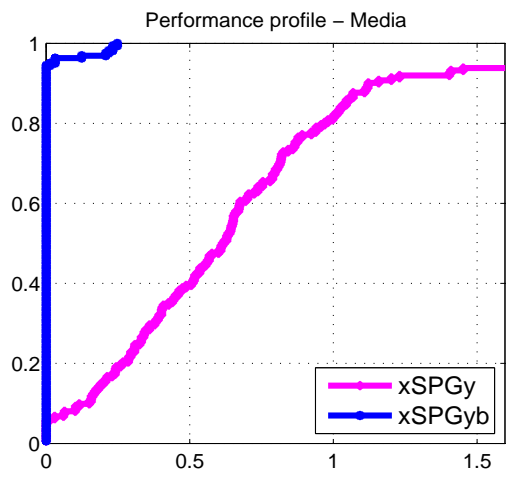

(a) Aleatórios

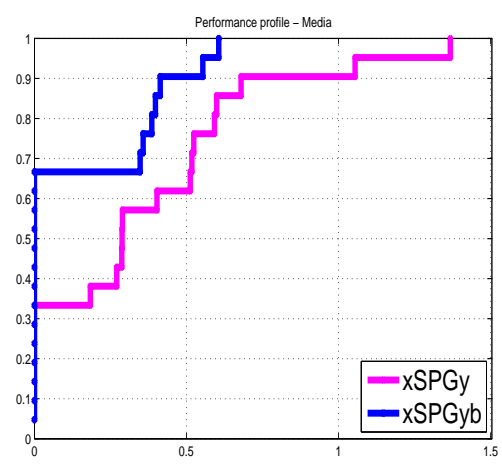

(b) Moré e Toraldo

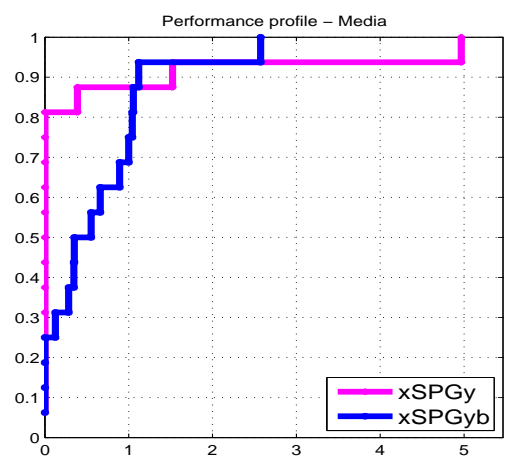

(c) Maros e Mezsaros

Figura 4.9: $(x S P G y) \times(x S P G y b)$

Temos então que o esquema xSPGyb foi mais eficente nas duas primeiras classes de problemas, embora isso não se reflita nos problemas Maros e Mezsaros. Como entre as classes que este método obteve mais sucesso estão os problemas de Moré e Toraldo, é razoável afirmar que o esquema xSPGyb é mais eficiente.

Refletindo sobre o método SPG puro, temos que para poder reproduzir o seu comportamento, o ponto atual precisa ser construído a partir do ponto anterior, formando uma sequência. Entretanto, isto não ocorre com a sequência de pontos $\left\{y^{k}\right\}$ ( $y^{k}$ não depende de $y^{k-1}$, e sim de $x^{k}$ e $v^{k}$ ) e portanto, da forma como estamos escolhendo o ponto candidato a $x^{k+1}$, nunca conseguimos reproduzir o método SPG original. Porém, na sequência de pontos $\left\{x^{k}\right\}, x^{k}$ é definido a partir de $x^{k-1}$. Logo, se escolhemos

$$
\tilde{x}=\operatorname{Proj}_{C}\left(x^{k}-\xi_{s p g} \nabla f\left(x^{k}\right)\right)
$$


com

$$
\xi_{\text {spg }}:=\frac{\left\langle x^{k}-x^{k-1}, x^{k}-x^{k-1}\right\rangle}{\left\langle x^{k}-x^{k-1}, \nabla f\left(x^{k}\right)-\nabla f\left(x^{k-1}\right)\right\rangle} .
$$

é possível, sempre que o passo $x^{k}$ for aceito repetidamente, imitar o método SPG descrito em [4], que é um método muito conhecido e usado na área de otimização por sua eficiência.

Sendo assim, como no caso de projeção a partir de $y^{k}$, podemos fazer uma busca para determinar o passo $\rho$. Então, podemos construir as seguintes versões do nosso método:

- xSPGx: $\tilde{x}$ com passo SPG definido por (4.8), sem busca, e $L_{a}$ definido pelo critério (4.2);

- $\mathrm{xSPGx}-2$ : $\tilde{x}$ com passo SPG definido por (4.8), sem busca, e $L_{a}$ definido pelo critério $\phi_{k+1}^{*} \geq f\left(x^{k+1}\right)$;

- xSPGxb: $\tilde{x}$ com passo SPG definido por (4.8), com buscas, e $L_{a}$ definido pelo critério (4.2);

- xSPGxb-2: $\tilde{x}$ com passo SPG definido por (4.8), com buscas, e $L_{a}$ definido pelo critério $\phi_{k+1}^{*} \geq f\left(x^{k+1}\right)$.

Abaixo, apresentamos os gráficos de perfil de desempenho para estas versões do nosso método: 


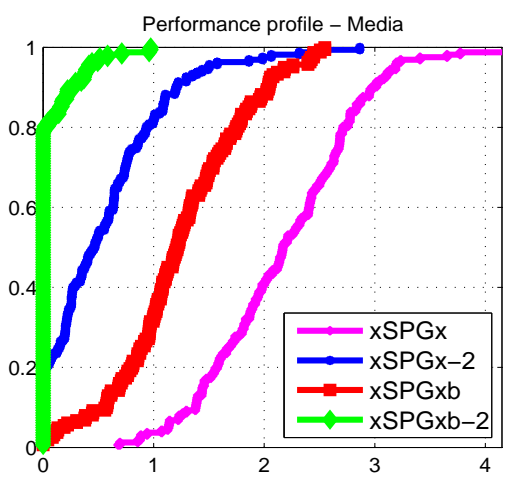

(a) Aleatórios

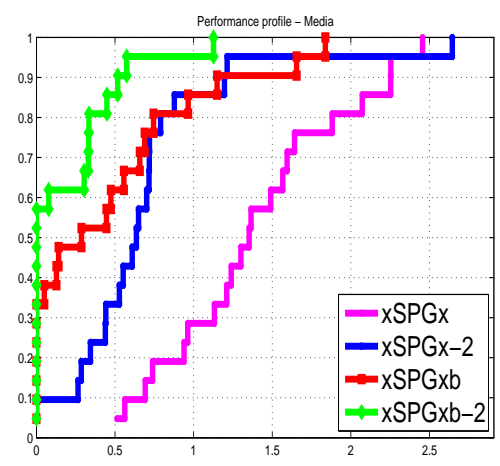

(b) Moré e Toraldo

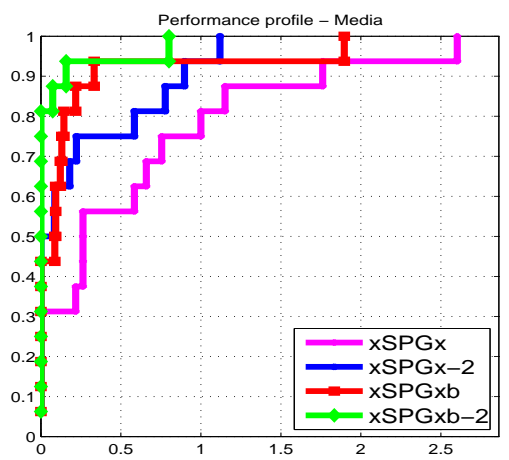

(c) Maros e Mezsaros

Figura 4.10: Métodos com projeção em relação a $x^{k}$ e passo $\xi_{\text {spg }}$

Nos três grupos de problemas o esquema xSPGxb-2 parece ser mais eficiente. Para visualisar melhorar o comportamento deste método refizemos o gráfico comparando este método com o método xSPGxb, que parece ser o segundo mais eficiente, particularmente nos problemas não aleatórios. 


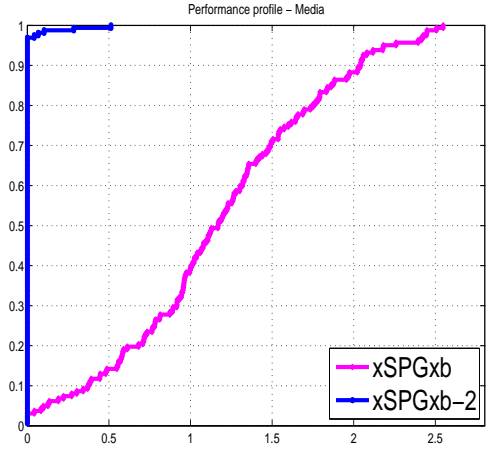

(a) Aleatórios

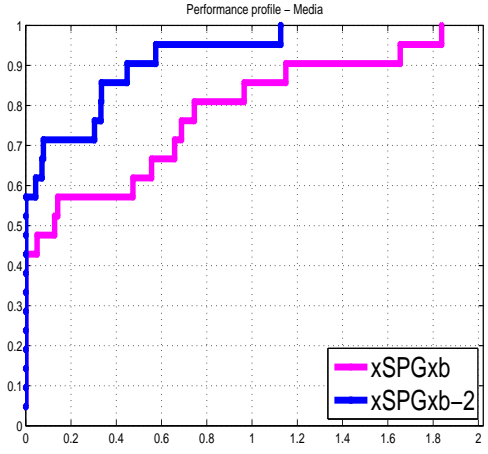

(b) Moré e Toraldo

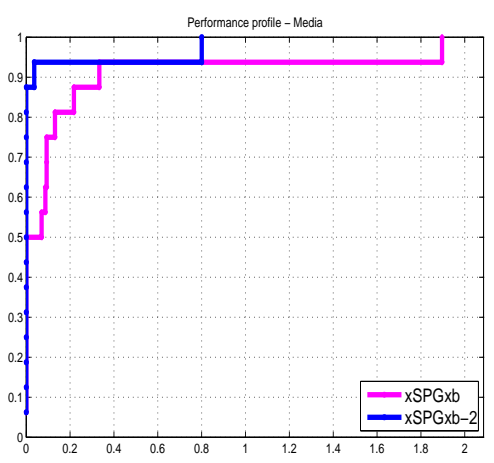

(c) Maros e Mezsaros

Figura 4.11: $(x S P G x b) \times(x S P G x b-2)$

Claramente podemos concluir que o método xSPGxb-2 é mais eficiente. Note, por exemplo, que para resolver cerca de $60 \%$ dos problemas aleatórios o esquema xSPGxb trabalhou mais que o dobro do outro método. Nos próximos gráficos comparamos este método com a melhor versão do método que usou o passo $\xi_{\text {spg }}$ porém a projeção foi feita a partir do ponto $y^{k}$. 


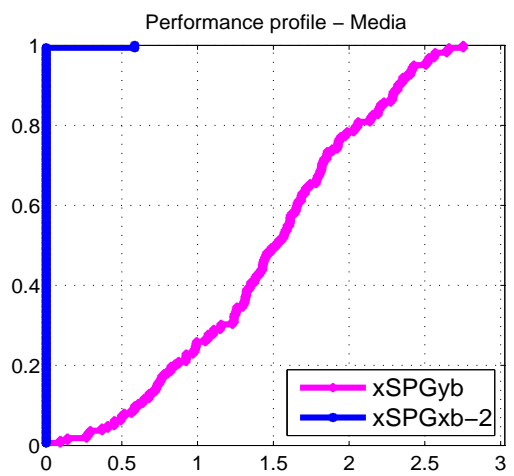

(a) Aleatórios

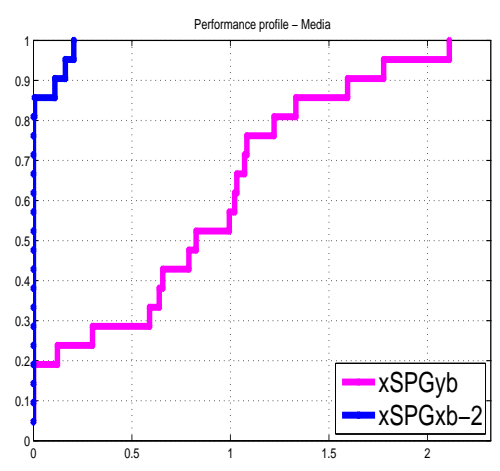

(b) Moré e Toraldo

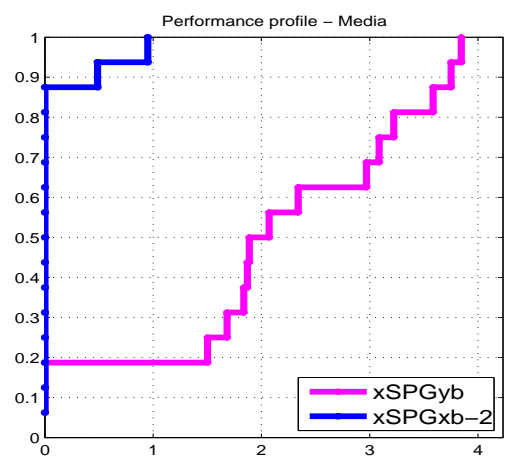

(c) Maros e Mezsaros

Figura 4.12: $(x S P G y b) \times(x S P G x b-2)$

Certamente podemos constatar que o melhor esqeuma é o xSPGxb-2. Observe, por exemplo, que para resolver a maioria dos problemas do primeiro e do terceiro grupo o procedimento xSPGyb trabalhou mais que o dobro do outro esquema. Este é o resultado que esperávamos, uma vez que fazendo projeções a partir de $x^{k}$ temos chance de reproduzir o comportamento do método SPG.

Abaixo comparamos o melhor destes dois métodos com um método idêntico exceto pela tentativa do passo $\theta \geq \alpha_{A T}$ para determinar $y^{k}$, este método representamos pela sigla xSPGxb-yb-2. 


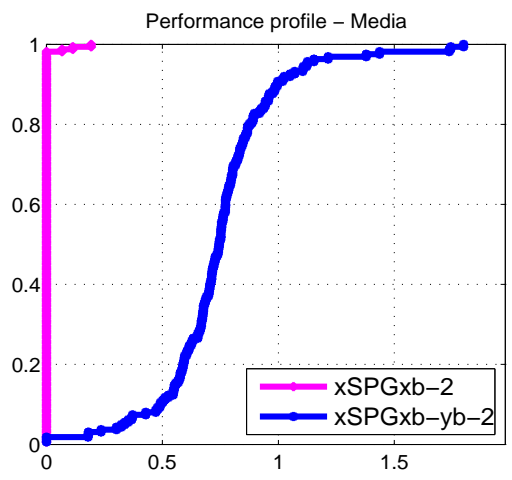

(a) Aleatórios

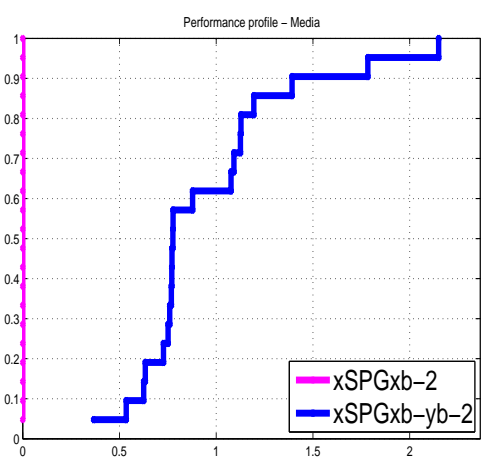

(b) Moré e Toraldo

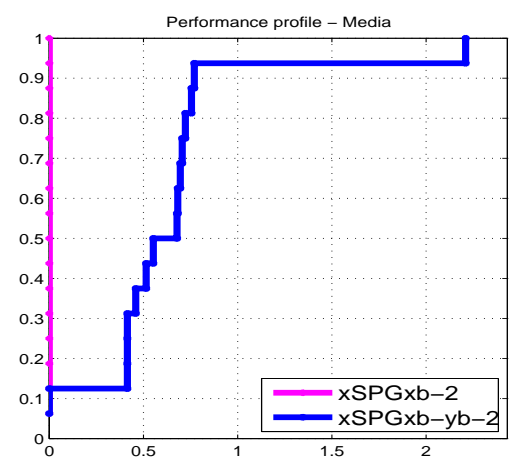

(c) Maros e Mezsaros

Figura 4.13: $(x S P G x b-2) \times(x S P G x b-y b-2)$

Como ocorreu nos métodos que usam o passo $\xi=\frac{1}{L_{a}}$, esta busca para determinar um passo $\theta \geq \alpha_{A T}$ na escolha de $y^{k}$ não melhora a eficiência do método. Sendo assim, podemos considerar que o melhor método com passo $\xi_{s p g}$ é o método que faz uma busca para determinar o tamanho do passo $\rho$, a projeção é feita a partir de $x^{k}$ e a desigualdade $\phi_{k+1}^{*} \geq f\left(x^{k+1}\right)$ é usada como critério de aceitação para $L_{a}$ (xSPGxb-2). Abaixo, comparamos este procedimento com o método ótimo de Nesterov descrito no capítulo anterior: 


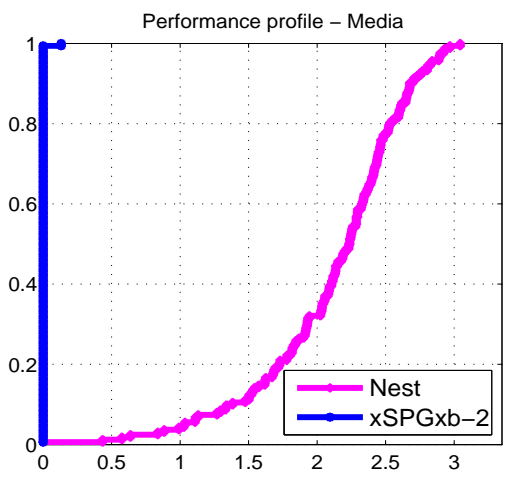

(a) Aleatórios

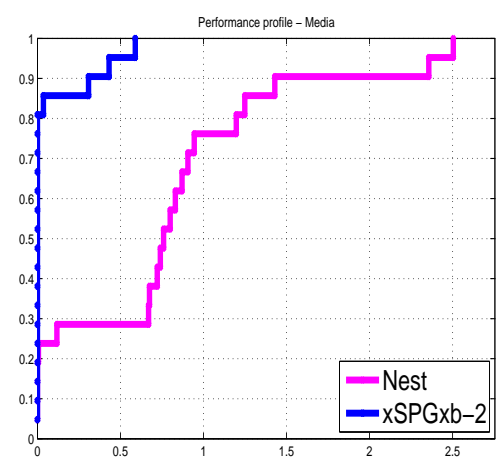

(b) Moré e Toraldo

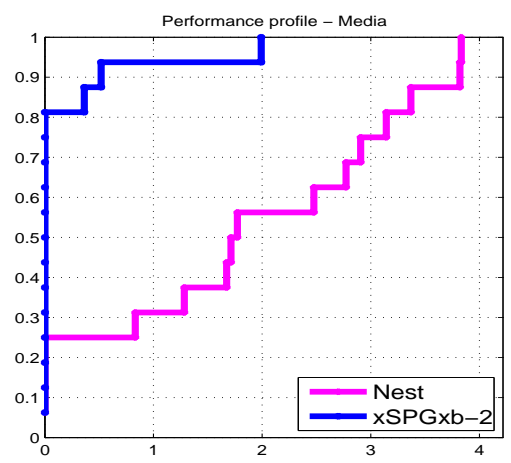

(c) Maros e Mezsaros

Figura 4.14: (Nest) $\times(\mathrm{xSPGxb}-2)$

Nas três classes de problemas, esta versão do nosso método foi acima de $80 \%$ mais eficiente que o método de Nesterov. Note novamente, que para resolver grande parte dos problemas da primeira e terceira classe, Nest precisou trabalhar mais que o dobro que o outro método.

Sendo assim, podemos concluir que com as liberdades de escolha que nosso método possibilita, podemos construir duas versões mais eficientes que o método ótimo de Nesterov. Para ficar claro qual das nossas versões é a melhor, exibimos os seguintes gráficos: 


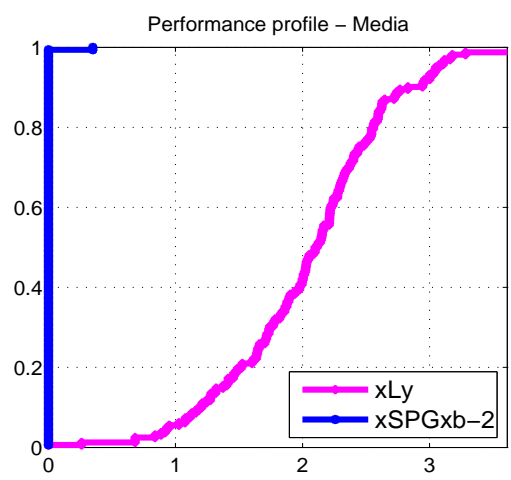

(a) Aleatórios

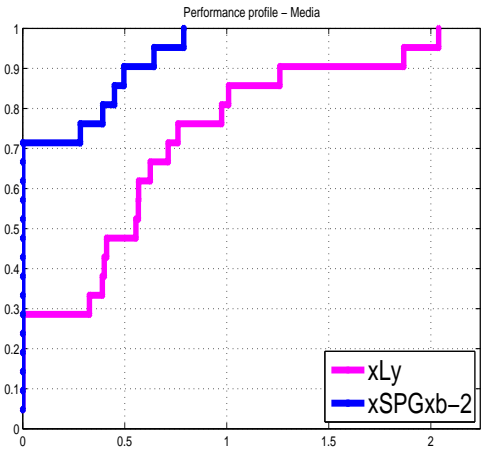

(b) Moré e Toraldo

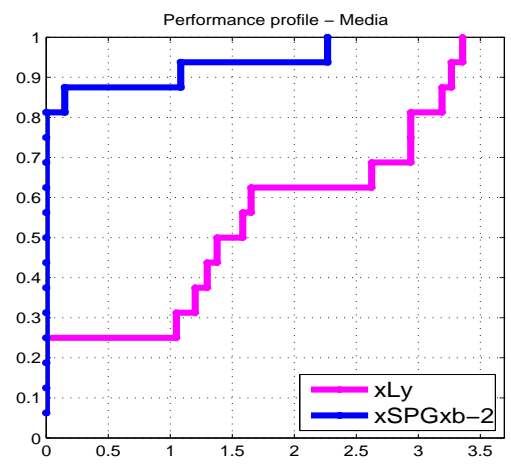

(c) Maros e Mezsaros

Figura 4.15: $(x L y) \times(x S P G x b-2)$

Observamos que nossa melhor versão é a que usa o passo $\xi_{s p g}$, uma vez que foi mais eficiente em todas as comparações e sempre realizou uma quantidade menor de trabalho para resolver todos os problemas. Em grande parte dos problemas esta diferença é de até uma ordem de grandeza menor. Acreditamos que a superioridade do esquema xSPGxb-2 ocorra pois esta versão pode em muitas iterações consecutivas imitar o método SPG puro, porém com a garantia de otimalidade. 


\section{Considerações Finais}

Neste trabalho formulamos um novo método ótimo para resolver problemas de otimização convexa com restrições. Partindo dos trabalhos de Nesterov e Auslender e Teoublle, desenvolvemos um algoritmo que usa a distânia euclidiana e que é capaz de estimar a constante de Lipschitz do gradiente da função objetivo. Além disso, nosso método possui ampla liberdade de escolher novos pontos buscando obter um decréscimo maior no valor da função objetivo resultando em implementações eficientes.

Entretanto, o procedimento apresentado nesta tese e outros métodos ótimos para problemas com restrições existentes não usam o parâmetro de convexidade forte da função objetivo. Durante o doutoramento, tivemos várias discussões sobre estes métodos com os professores Clóvis Caesar Gonzaga e Elizabeth Wegner Karas. Destes encontros surgiram muitas sugestões para este trabalho. Além disto, desenvolvemos um método ótimo para problemas com restrições, que permite o uso do parâmetro de convexidade forte da função objetivo [8].

O método desenvolvido nesta tese permite incorporar idéias de bons procedimentos permitindo acelerar a convergência do algoritmo. Como trabalho futuro, pretendemos avaliar o desempenho do novo método ao incorporar nele as idéias de métodos quasi-Newton com memória limitada. 


\section{Referências Bibliográficas}

[1] A.Auslender, P. J. S. Silva, And M. Teboulle, Nonmonotone projected gradient methods based on barrier ande euclidean distances, SIAM J. OPTIM, (2006). 33, 37

[2] A. Auslender And M. TeBoulle, Interior gradient and proximal methods for convex and conic optimization, Computacional Optimization and Applications, (2007). 2, 40, 46

[3] D. P. Bertsekas, A. Nedié, And A. E. Ozdaglar, Convex Analysis and Optimization, Athena Scientific, 1963. 3, 6, 8

[4] E. G. Birgin, J. M. Martinez, And M. Raydan, Spectral projected gradient methods, Encyclopedia of Optimization, C, (2009), pp. 3652-3659. 75, 76, 79

[5] Y. Dai And R. Fletcher, Projected barzilai-borwein methods for large-scale box-constrained quadratic programming, Numerische Mathematik, (2005). 63

[6] E. D. Dolan AND J. J. MoRÉ, Benchmarking optimization software with performance profiles, Mathematical Programming, (2002), pp. 201-213. 64

[7] C. C. Gonzaga And E. W. Karas, Optimal steepest descent algorithms for unconstrained convex problems: fine tuning nesterov's method, tech. rep., Federal University of Paraná, 2008. 1, 52

[8] C. C. Gonzaga, E. W. Karas, and D. R. Rossetto, An optimal algorithm for constrained differentiable convex optimization, submitted, (2011). 87

[9] A. Griewank, On automatic differentiation, in In Mathematical Programming: Recent Developments and Applications, Kluwer Academic Publishers, 1989, pp. 83-108. 65

[10] A. Iusem, Método de Ponto Proximal em Otimização, Instituto de Matemática Pura e Aplicada - CNPq. Livro do 20 Colóquio Brasileiro de Matemática, 1995. 39

[11] A. Izmailov And M. Solodov, Otimização - Volume 1, IMPA, 2005. 3

[12] J. Moré And G. TORALdO, On the solution of large quadratic programming problems with bound constraints, SIAM J. Optimization, (1991). 64

[13] Y. NeSterov, A method for unconstrained convex minimization problem with the rate of convergence $o\left(1 / k^{2}\right)$, Doklady AN SSSR (translated as Soviet Math Docl), (1983), pp. $543-547.1$ 
[14] - Introductory Lectures on Convex Optimization. A basic course, Kluwer Academic Publishers, Boston, 2004. 3, 6, 8, 12, 13

[15] — Gradient method for minimizing composite objective function, CORE Discussion Paper, (2007). 1, 2, 5, 15, 33, 34, 35, 46

[16] P. J. S. SiLva, Método de ponto proximal e separadores, Master's thesis, Universidade de São Paulo, 1997. 3, 37, 40, 67 